\title{
Generalization Bounds and Algorithms for Learning
} to Communicate over Additive Noise Channels

\author{
Nir Weinberger \\ The Viterbi Faculty of Electrical and Computer Engineering \\ Technion - Israel Institute of Technology \\ Technion City, Haifa 3200004, Israel \\ nirwein@.technion.ac.il
}

\begin{abstract}
An additive noise channel is considered, in which the distribution of the noise is nonparametric and unknown. The problem of learning encoders and decoders based on noise samples is considered. For uncoded communication systems, the problem of choosing a codebook and possibly also a generalized minimal distance decoder (which is parameterized by a covariance matrix) is addressed. High probability generalization bounds for the error probability loss function, as well as for a hinge-type surrogate loss function are provided. A stochastic-gradient based alternatingminimization algorithm for the latter loss function is proposed. In addition, a Gibbs-based algorithm that gradually expurgates an initial codebook from codewords in order to obtain a smaller codebook with improved error probability is proposed, and bounds on its average empirical error and generalization error, as well as a high probability generalization bound, are stated. Various experiments demonstrate the performance of the proposed algorithms. For coded systems, the problem of maximizing the mutual information between the input and the output with respect to the input distribution is addressed, and uniform convergence bounds for two different classes of input distributions are obtained.
\end{abstract}

\section{Index Terms}

additive noise channels, alternating optimization algorithm, entropy estimation, expurgation, generalization bounds, hinge loss, Gibbs algorithm, minimal distance decoding, mismatch decoding, statistical learning, stochastic gradient descent.

This research was supported by the MIT-Technion fellowship and the Viterbi scholarship, Technion, Israel Institute of Technology. The material in this paper was presented in part at IEEE International Symposium on Information Theory (ISIT) 2020. 


\section{INTRODUCTION}

The additive noise channel is one of the simplest and most widely applicable models in communication and information theory. The channel output $Y \in \mathbb{R}^{d}$ in this model is given by

$$
Y=X+Z
$$

where $X \in \mathbb{R}^{d}$ is the input (which is almost always restricted in some way, e.g., to have finite power), and $Z \in \mathbb{R}^{d}$ is the noise, which is assumed to be statistically independent of the input $X$. As is natural and well-established [17], [64], the optimization of a communication system for this channel depends on the probability distribution of the noise: First, via a proper choice of the decoder which minimizes the error probability for a given codebook, and second, via the proper design of the codebook itself in case of uncoded transmission, or the capacity achieving input distribution in case of coded transmission. The conventional model further assumes that $Z$ is Gaussian, which is typically justified by physical phenomena such as thermal noise at the receiver [93], as well as the central-limit theorem which implies that the accumulated noise from a large number of sources tends to be Gaussian. If such a model is accurate, then the parameters of the noise distribution ${ }^{1}$ can be estimated from noise samples obtained by the receiver when the transmitter is silent, and then used to optimize the system. This method similarly works whenever the noise density is parametric of sufficiently low dimension. Most often (e.g. [48]), this includes the design of a training sequence $\left\{x_{\mathrm{tr}, i}\right\}_{i=1}^{T_{\mathrm{tr}}}$ which is transmitted over the channel over $T_{\mathrm{tr}}$ uses of the channel $[1]$, and the receiver which knows this sequence uses it to estimate the parameters of the distribution of $Z$.

Nonetheless, in more complex scenarios encountered in practice - for example in massive multiple-input multipleoutput (MIMO) systems [75] or in ultra low-latency [110] communication - the class of possible noise distributions may be too rich to allow efficient and faithful parameter estimation under the system constraints, or may be essentially nonparametric to begin with. Following the statistical learning paradigm [49], [105], [116], a possible approach is to define a hypothesis class for the encoder (codebooks) and the decoder, and use the samples of the noise to select a member of the class. The selected decoder in this class is most assuredly mismatched to the noise distribution and is not a maximum-likelihood decoder for the selected codebook. However, the great advantage of this approach is that the class of codebooks and decoders may only include encoders/decoders which are feasible to implement, by design.

In this paper, we focus on learning aspects of this problem, and specifically on the convergence rates of generalization bounds as a function of the number of noise samples. Nonetheless, we mainly ignore computational complexity and scalability aspects of the algorithms proposed to perform such learning. Furthermore, as the noise samples are usually only available at the decoder side, we also mainly sidestep the problem of communicating the choice of codebook back to the encoder $2^{2}$ Rather instead, our goal is to explore the statistical learnability of coding

\footnotetext{
${ }^{1}$ The covariance matrix, or just the variance in case of white noise.

${ }^{2}$ This problem is inconsequential in offline design, or when a reliable feedback link of large capacity exists. Analysis of limited feedback makes the model more complex and is an important topic for further research.
} 
problems in a distilled and basic form, and highlight basic properties and challenges.

\section{A. Contributions and Outline}

Our contributions are of two types - for uncoded and coded systems. For both types we assume that the learner has $n$ independent samples of the noise $Z$. For uncoded systems, one-shot use of (1) is assumed, and based on the noise samples, the learner is required to design a codebook of $m$ codewords, and possibly select a decoder from a given class in order to minimize a prescribed loss function. It is tacitly assumed that $m$ is reasonably small, so that the encoder can memorize all codewords (such codebooks are sometimes referred to as multi-dimensional constellations). For this setup, we show that:

1) Section III If the class of decoders is comprised of all possible minimal distance decoders with respect to (w.r.t.) the Mahalanobis distance (parameterized by a covariance matrix), and if the loss function is the standard error probability, then the generalization error of selecting a codebook of $m$ codewords scales as $\tilde{O}\left(m \sqrt{\frac{d}{n}}\right)$ with high probability. The result is an error bound which is uniformly applicable to all codebooks and decoders, and thus for any learning algorithm. Specifically, it holds for the empirical risk minimization (ERM) algorithm. At the same time, to the best of our knowledge there is no efficient ERM algorithm for this problem. We also show that the $\frac{1}{\sqrt{n}}$ dependency is tight.

2) Section IIIf If the loss function is replaced by a hinge-type surrogate loss function, which unlike the standard error probability loss function is a continuous function of the codebook and decoder covariance matrix, then the generalization error scales at an improved rate of $\tilde{O}\left(\sqrt{\frac{(d \vee m) d}{n}}\right)$ with high probability. Moreover, a heuristic alternating optimization algorithm is proposed for this problem, and a stochastic gradient descent (SGD) variant of this algorithm is outlined.

In Section IV, we consider a setup in which a large static codebook of $m_{0}>m$ codewords is given, and the noise samples are used to dynamically expurgate this codebook by removing "bad" codewords which cause large error probability (the decoder is assumed to be a standard minimal distance decoder). Finding the optimal subset of codewords is a combinatorial optimization optimization problem, which is computationally heavy to solve in general. We thus propose a randomized Gibbs algorithm for this scenario, which gradually removes codewords with some randomness. The use of this Gibbs algorithm allows us to obtain a bound on its average generalization error, which follows directly from an information-theoretic stability analysis of learning algorithms [94], [128]. The bound scales as $O\left(\sqrt{\frac{T \beta}{n} \wedge \frac{T \beta^{2}}{4 n^{2}}}\right)$ where $T$ is the number of steps of the algorithm; the error probabilities conditioned on each codeword are re-computed after each step before expurgating another subset of $k=\frac{m_{0}-m}{T}$ codewords; and $\beta$ is an inverse-temperature parameter which controls the balance between greedy and completely random expurgation. We also state a high probability generalization bound of order $\tilde{O}\left(\frac{\sqrt{T} \beta}{n}+\frac{1}{\sqrt{n}}\right)$ which is a direct implication of the uniform-stability property [13] of the Gibbs algorithm, and the recent results of [33], [34]. The choice of $T$ and $k$ is dictated by the available computational power, whereas the value of $\beta$ may be arbitrary optimized. 
For coded systems, we focus in Section $\mathrm{V}$ on the problem of finding the input distribution which maximizes the mutual information $I(X ; X+Z)$, or, equivalently, the output differential entropy $h(X+Z)$. The use of the mutual information as a performance measure may be justified by the theoretical existence of universal decoding rules which are able to achieve communications rates arbitrarily close to the mutual information despite lack of knowledge of the noise distribution [79] (see also the survey in [65]) $]^{3}$ Evidently, this problem is closely related to the problem of differential entropy estimation (surveyed in [120]; see also [46] and references therein for a more recent account), but here the goal is to maximize the entropy, rather than to estimate it. This can be achieved by showing a empirical convergence of an estimator $h_{n}(X+Z)$ based on the noise samples which is uniform in allowed class input distributions. The methods used here to achieve this is to control the difference between differential entropies by some statistical distance between distributions, and specifically, between the true and empirical distributions. We thus consider two types of sets of conditions and show that:

1) If the set of input distributions is regular in the sense defined in [92], has finite second moment, and has absolutely bounded differential entropy, and if the norm of the noise is sub-Gaussian, then with high probability $\left|h(X+Z)-h\left(X+\hat{Z}_{n}\right)\right|=\tilde{O}\left(n^{-1 /(d \vee 4)}\right)$ uniformly for all possible input distributions, where $\hat{Z}_{n}$ is the empirical measure of the noise samples.

2) If the support of $X$ is fixed to $m$ points $\left\{x_{1}, \ldots, x_{m}\right\} \subset \mathbb{R}^{d}$ (an atomic distribution), and so the input distribution is determined by weights $\left\{a_{j}\right\}_{j \in[m]}$ such that $a_{j}=\mathbb{P}\left[X=x_{j}\right]$, then a similar uniform high probability bound can be obtained using a plug-in kernel density estimator (KDE) for the noise density. The final convergence rates of the uniform error in differential entropy depend on smoothness assumptions on the noise densities. For example, for Lipschitz balls [46] of smoothness parameter $s \in(0,2]$ it is given by $O\left(n^{-s /(s+d)}\right)$. Importantly, this rate is dominated by the entropy estimation error, and the additional error due to the requirement for uniform convergence scales as $\tilde{O}\left(\sqrt{\frac{m}{n}}\right)$ which is typically negligible.

As evident, the convergence rates in this problem scale exponentially with the dimension, which stems from the fact that at worst case, the support of the noise distribution may lie in a $d$-dimensional space. Possible ways to obtain faster convergence rates are discussed, along with a short summary and other open problems in Section VII. Proofs are relegated to Appendix A, parameters used in experiments are summarized in Appendix B, and technical details regarding the implementation of the Gibbs algorithm are provided in Appendix C.

\section{B. Motivation and Context}

The remarkable practical success of learning methods of deep neural networks (DNN) [41] architectures in an assortment of tasks has recently motivated various researchers to consider learning-based design of communication systems [88]. As surveyed in [44], [56], this idea is not completely new and was proposed as early as the 1990s

\footnotetext{
${ }^{3}$ The exact maximal achievable rate using a fixed-structure mismatched decoder ("mismatch capacity") is unknown to date [103], [109]. A more complicated alternative to the problem studied here, is to explore generalization bounds which aim to maximize known lower bounds on the mismatch capacity (for example, the one known as the LM rate [19], [54], [82]).
} 
by several authors. The approach to this problem is coarsely categorized into either one of two types [88]. The first type [16], [87], [122] is motivated by the expectation to utilize the powerful computational resources available for implementation of DNN and their efficient training methods in order to achieve performance competitive to expert-designed systems. Various recent works have considered this approach, e.g., for random and polar codes [44], for an unfolded belief propagation algorithm [87], for MIMO communication [102], for low-latency codes [58], [61] motivated by 5th generation standard [110], for feedback-based communication [60], and for interactive communication [101].

The second type is closer in spirit to the one taken in this paper, and follows the general learning-theoretic methodology to refrain from parametric modeling of the data probability distribution [49], [105], [116]. Rather instead, a hypothesis class is assumed for the required statistical inference goal (e.g., classification, regression), and data is used to select an optimal hypothesis from this class based on the available data. The application of this approach in communication-theory related problems is most suitable in scenarios for which channel modeling is difficult or inaccurate. This includes, e.g., interference, jamming signals non-linearity [104] finite-resolution quantization (see [88], [121]). The entire end-to-end system is considered as a single DNN, encompassing the encoder operation, the operation and randomness of the channel, and the decoder operation, and is then typically trained in an unsupervised manner as an autoencoder [41]. This approach was applied to mutli-user detection in code-division multiple-access systems [85], and, more recently, to molecular communication systems [31]. In [131], a receiver architecture including a processing of the input followed by nearest neighbor decoding rule was proposed. The optimal output processing was identified to be a regression problem, and it was proposed to use the data in order to choose the processing function to maximize a generalized mutual information (under Gaussian codebooks).

In fact, such an approach seems to be also useful in case a parametric modeling of the channel is reasonably accurate, but too complex to be utilized, as the dimension of the parameter vector is exceedingly high (e.g., in massive MIMO systems [75]). As mentioned above, parameter estimation is a common practice to provide channel state information (i.e., noise distribution) to the decoder, and is usually carried by transmitting a designated training sequence. However, for high-dimensional parameters, insufficient training time will lead to large variance of the estimator, and coping this by dimensionality reduction may lead to a large bias. Thus a good variance-bias trade-off cannot be achieved. Moreover, the estimation step is only secondary to the ultimate goal of reliable decoding of the data with low error probability. In other words, decoder selection based on parameter estimation is a specific, indirect, way of selecting a decoder based on noise samples. A more general and direct way is to allow any selection rule of the decoder based on the noise samples.

Nonetheless, communication theory enjoys a rich expert knowledge in optimizing its main components, and many practical communication channels do have reasonably faithful parametric representation, which is widely and successfully used in practice (e.g., [5], [51], [93], [111]). To overcome this high bar and demonstrate the viability of the learning-based approach, research has focused on obtaining improved or comparable performance (with improved complexity) to classical communication-theoretic methods. To obtain this goal, they utilize sophisticated 
DNN architectures, which exhibit both remarkable approximation ability [52] as well as generalization to out-ofsample data. To date, however, the generalization capabilities and the efficiency of training algorithms such as SGD of DNNs are not fully understood, and challenge general principles in statistics and learning (e.g., [8]). Moreover, even with refined understanding, the application of any learning methods to a communication problem raises additional questions: What is the richness of the decoder hypothesis classes? How does the generalization error is affected by the structure of the code and its data rate? What are useful and proper loss functions? How to properly regularize the learning process? In this paper, we focus on a stylized and basic version of this problem, with the prospect that the opportunities and challenges associated with using learning-theoretic methods for communication systems are illuminated by the analysis of this basic model.

\section{Other Related Work}

The analysis of decoders which are not fully optimized to the channel statistics distribution is typically studied under the framework of mismatch decoding. Sharp characterization of the capacity and other fundamental limits of such decoders is a notoriously difficult and extensively studied open problem in information theory [4], [21], [39], [55], [63], [65], [82], [103], [108], [109]. In this model, however, the encoder and decoder are assumed to be completely fixed in advance and are not selected in any way. By contrast, in this work we focus on the selection of a decoder from a class of decoders using samples of the noise, and specifically, how well this can be done in a way which generalizes well to out-of-sample noise.

While being somewhat new to channel coding problems, empirical design of encoders is a common practice for lossy source coding problems, usually via the celebrated Lloyd-Max algorithm [43]. Learning-theoretic analysis of empirically designed quantizers was carried out during the 1990's and onward [2], [6], [70]-[73], [83] |4 Among various results, it was established that a distortion redundancy of $\epsilon$ is achievable with $n=\tilde{O}\left(\frac{d^{3} m^{1-2 / d}}{\epsilon^{2}}\right)$ samples, where here $m$ is the size of quantization codebook (number of reproduction points). The seemingly peculiar dependency on $m$, and the optimal minimax tightness of its dependency on $n$ is discussed and established in [6, Thm. 2] (see also [1]). More recently, this setup was generalized to coding from a general Hilbert space to a finite-dimensional space, with linear [76] and non-linear [66] mappings. Beyond vector quantization (or clustering), this framework is general enough to also include principal component analysis, non-negative matrix factorization, dictionary learning [113], and modern unsupervised representation learning techniques, such as autoencoders. Given that channel coding (communication) bears both similarities and differences with source coding (mainly quantization), understanding learning-theoretic questions for channel coding seems feasible, but also requires dedicated effort to handle the unique features of the latter.

A learning approach that avoids channel modeling and estimation, may be contrasted with the universal decoding approach prevalent in information theory [20], [65]. Similarly to the learning-based approach, universal methods avoid explicit estimation of the channel, and aim to find a single decoding rule which does not depend on the

\footnotetext{
${ }^{4}$ As an exception to this, a channel coding related problem was considered in [80], which mainly focused on impossibility results.
} 
unknown channel statistics, yet it is simultaneously nearly optimal for any channel in the class (in a sense which requires explicit definition, e.g., as in [32]). The universal approach is highly applicable on the theoretical side, but bares some challenges from the practical side $5^{5}$ The key problem is that in most problems the universal decoder obtained is required to compute a metric for all codewords in the codebook, which is typically infeasible due to the large number of codewords, e.g., [123]. This is true even if the code is structured, e.g., a linear or a convolutional code, such that optimal or efficient decoding rules, e.g., Viterbi's decoder [119] or a belief propagation [99] decoder, can be efficiently implemented when the channel statistics is available. Furthermore, it was shown in [81] that a universal decoder which aims to compete with a family of low complexity decoders will need to be much more complex than each of those decoders. Loosely speaking, this is an inherent difficulty for any universal approach because in some sense, universal decoders implicitly learn the channel statistics and decode the message at the same time. Hence, they cannot rely on likelihood information that is obtained separately from each channel output, not even when the channel is known to be memoryless. Similar situation occurs in lossy source coding, for which [127, Sec. V] states that "low computational complexity is achievable only at the expense of yielding a nonoptimal distortion" (mainly regarding such practical methods which are based on approximate string matching). By contrast, in a learning-based approach, the class of decoders may be restricted in advance to feasible decoders, and the available data may then be used to choose the best one from the given class.

\section{Main Notation Conventions}

We mainly use standard notation or define it before its first use, and here only highlight main conventions. The standard Euclidean norm for $x \in \mathbb{R}^{d}$ is denoted by $\|x\|$. The operator norm for a matrix $S \in \mathbb{R}^{d \times d}$, viewed as an operator from $\mathbb{R}^{d}$ to $\mathbb{R}^{d}$ (with both spaces equipped with the Euclidean norm), is denoted by $\|S\|_{\text {op }}$. The maximal eigenvalue (resp. minimal) of a symmetric matrix $S$ is denoted by $\zeta_{\max }(S)\left(\operatorname{resp} . \zeta_{\min }(S)\right)$. The $d$-dimensional diagonal matrix whose diagonal entries are given by $v \in \mathbb{R}^{d}$ is denoted by $\operatorname{diag}(v) \in \mathbb{R}^{d \times d}$. The zero-centered Euclidean ball of radius $r$ is denoted by $\mathbb{B}^{d}(r):=\left\{x \in \mathbb{R}^{d}:\|x\| \leq r\right\}$ and the unit sphere is denoted by $\mathbb{S}^{d-1}:=$ $\left\{x \in \mathbb{R}^{d}:\|x\|=1\right\}$. For $n \in \mathbb{N}_{+}$, the set $\{1,2, \ldots, n\}$ is denoted by $[n]$. The cardinality of a finite set $A$ is denoted by $|A|$. The Hamming distance between $x=\left\{x_{1}, \ldots, x_{d}\right\}, y=\left\{y_{1}, \ldots, y_{d}\right\} \in \mathbb{R}^{d}$ is denoted by $\mathrm{d}_{\mathrm{H}}(x, y):=$ $\left|\left\{i \in[d]: x_{i} \neq y_{i}\right\}\right|$. The minimum (resp. maximum) of $a, b \in \mathbb{R}$ is denoted by $a \wedge b$ (resp. $a \vee b$ ). The $(m-1)$ dimensional probability simplex is denoted by $\mathbb{A}^{m-1}:=\left\{\boldsymbol{a}=\left(a_{1}, \ldots, a_{m}\right): \sum_{j} a_{j}=1, a_{j} \geq 0\right\}$. The indicator of an event $\mathcal{A}$ is denoted by $\mathbb{1}\{\mathcal{A}\}$. As a general rule, random variables or vectors will be denoted by capital letters and specific values they may take will be denoted by the corresponding lower case letters. Statistical independence between random variables $X, Y$ is denoted by $X \Perp Y$. Equality of the probability distributions of $X, Y$ is denoted by $X \stackrel{d}{=} Y$. The Kullback-Leibler (KL) divergence between the probability distributions $\mu$ and $\tilde{\mu}$ is denoted by $\mathrm{d}_{\mathrm{KL}}(\mu \| \tilde{\mu})$. Integrals of probability densities $f$ (which absolutely continuous w.r.t. Lebesgue measure $\lambda$ ) are taken w.r.t. the Lebesgue measure $\lambda$, and this is abbreviated as $\int f:=\int f \mathrm{~d} \lambda$. The set of Borel probability measures on

\footnotetext{
${ }^{5}$ In contrast to the remarkable practical success of universal methods is lossless data compression [132], [133].
} 
$\mathbb{R}^{d}$ is denoted by $\mathcal{P}\left(\mathbb{R}^{d}\right)$. For $\mu_{U}, \mu_{V} \in \mathcal{P}\left(\mathbb{R}^{d}\right)$, the $p$ th order Wasserstein distance is denoted by $W_{p}\left(\mu_{U}, \mu_{V}\right):=$ $\inf \mathbb{E}^{1 / p}\left(\|U-V\|^{p}\right)$ where $U \sim \mu_{U}$ and $V \sim \mu_{V}$, and where the infimum is taken over all couplings $\mu_{U V}$ (i.e., joint distributions which agree with the marginal measures of $U$ and $V$ ). A real random variable $X$ is called $\sigma$-sub-Gaussian whenever

$$
\sigma=\|X\|_{\psi_{2}}:=\inf \left\{t>0: \mathbb{E} \exp \left(X^{2} / t^{2}\right) \leq 2\right\}<\infty
$$

\section{LEARning Under AN ERRor Probability Loss Function}

\section{A. Problem Formulation}

Consider again the additive noise channel in $(1):$ The input is denoted by $X \in \mathbb{R}^{d}$, the output by $Y=X+Z \in$ $\mathbb{R}^{d}$ where $X \Perp Z \in \mathbb{R}^{d}$, and where for the purpose of matrix multiplication are all taken as column vectors. The distribution of $Z$ is denoted by $\mu$, and it is assumed that it is completely unknown to the designer of the communication system. Let $\|x-y\|_{S}:=\sqrt{(x-y)^{T} S(x-y)}$ be the Mahalanobis distance between $x, y \in \mathbb{R}^{d}$ which is parameterized by an inverse covariance matrix $S \in \mathbb{S}_{+}^{d}$ (where $\mathbb{S}_{+}^{d}$ is the positive-definite cone). The transmitter chooses a codebook $C=\left\{x_{j}\right\}_{j \in[m]}$ of size $|C|=m$ where $x_{j} \in \mathbb{R}^{d}$ for all $j \in[m]$, and at each use of the channel (1), chooses a codeword from $C$ uniformly at random to send over the channel. The decoder employs a nearest neighbor decoder w.r.t. $\|\cdot\|_{S}$, i.e., given a channel output $y$, the index of the decoded codeword is chosen as:

$$
\hat{j}(y) \in \underset{j \in[m]}{\arg \min }\left\|x_{j}-y\right\|_{S}
$$

Our choice of decoding rule of the form (3) is motivated by the fact that this is the optimal (maximum-likelihood) rule when $Z \sim N\left(0, S^{-1}\right)$, that is, whenever the noise distribution is Gaussian with covariance matrix $S^{-1}$. We stress, however, that we do not assume that the noise $Z$ is Gaussian, and so 3 is chosen for its simplicity, and, in general, will not be a maximum likelihood decoder. The decision region of the $j$ th codeword is $\left\{y:\left\|x_{j}-y\right\|_{S} \leq\right.$ $\left.\min _{j^{\prime} \in[m] \backslash\{j\}}\left\|x_{j^{\prime}}-y\right\|_{S}\right\}$ and the boundary of these decision regions belong to the set of hyperplanes defined by $\left\|x_{j}-y\right\|_{S}=\left\|x_{j^{\prime}}-y\right\|_{S}$ for all $j \neq j^{\prime}$.

The expected error probability (over the noise distribution) given that the $j$ th codeword was transmitted is then given by

$$
\begin{aligned}
\mathbf{p}_{\mu}(C, S \mid j) & :=\mathbb{E}_{Z \sim \mu}\left[\mathbb{1}\left\{\min _{j^{\prime} \in[m] \backslash\{j\}}\left\|x_{j}+Z-x_{j^{\prime}}\right\|_{S}<\|Z\|_{S}\right\}\right] \\
& =\mathbb{E}_{Z \sim \mu}\left[\mathbb{1}\left\{\min _{j^{\prime} \in[m] \backslash\{j\}}\left\|x_{j}-x_{j^{\prime}}\right\|_{S}^{2}+2\left(x_{j}-x_{j^{\prime}}\right)^{T} S Z<0\right\}\right],
\end{aligned}
$$

and the expected average error probability by

$$
\mathbf{p}_{\mu}(C, S):=\frac{1}{m} \sum_{j=1}^{m} \mathbf{p}_{\mu}(C, S \mid j)=\mathbb{E}_{Z \sim \mu}\left[\frac{1}{m} \sum_{j=1}^{m} \mathbb{1}\left\{\min _{j^{\prime} \in[m] \backslash\{j\}}\left\|x_{j}-x_{j^{\prime}}\right\|_{S}^{2}+2\left(x_{j}-x_{j^{\prime}}\right)^{T} S Z<0\right\}\right] .
$$


The learning goal related to this formulation is the use of noise samples to design either a codebook $C \in \mathcal{C} \subset\left(\mathbb{R}^{d}\right)^{m}$, or a decoder inverse covariance matrix $S \in \mathcal{S} \subset \mathbb{S}_{+}^{d}$, or both, with minimal expected error probability. Here, the set $\mathcal{C}$, for example, could represent an average power constraint $\mathcal{C}=\left\{C: \frac{1}{m} \sum_{j=1}^{m}\left\|x_{i}\right\|^{2} \leq r\right\}$ for some $r>0$, and the set $\mathcal{S}$ may represent a restriction on $\|S\|$ for some matrix norm.

Assuming that the codebook/decoder designer is provided with $n$ samples of the noise $Z=\left\{Z_{i}\right\}_{i=1}^{n} \stackrel{\text { i.i.d. }}{\sim} \mu$, the empirical average error probability (over the $n$ samples $\left\{Z_{i}\right\}$ of the noise) is given by

$$
\mathbf{p}_{\boldsymbol{Z}}(C, S)=\frac{1}{n} \sum_{i=1}^{n} \frac{1}{m} \sum_{j=1}^{m} \mathbb{1}\left\{\min _{j^{\prime} \in[m] \backslash\{j\}}\left\|x_{j}-x_{j^{\prime}}\right\|_{S}^{2}+2\left(x_{j}-x_{j^{\prime}}\right)^{T} S Z_{i}<0\right\} .
$$

Note that we may write the empirical average error probability as

$$
\mathbf{p}_{\boldsymbol{Z}}(C, S)=\frac{1}{n} \sum_{i=1}^{n} \ell\left(C, S, Z_{i}\right)
$$

where the loss function is given by

$$
\ell(C, S, z):=\frac{1}{m} \sum_{j=1}^{m} \ell_{j}(C, S, z)
$$

with

$$
\ell_{j}(C, S, z):=\mathbb{1}\left[\min _{j^{\prime} \in[m], j^{\prime} \neq j}\left\|x_{j}-x_{j^{\prime}}\right\|_{S}^{2}+2\left(x_{j}-x_{j^{\prime}}\right)^{T} S z<0\right] .
$$

Comparison to multiclass classification: Evidently, the problem here resembles multiclass classification, but as we next discuss, the problems are not equivalent. According to our formulation, the data of the learner are $n$ noise samples $\left\{z_{i}\right\}_{i=1}^{n}$. In multiclass classification, each data sample $z_{i}$ should be equipped with a label $j \in[m]$, and the goal of the learner is to output a classifier, whose performance is measured under the $0-1$ loss function, which equals 1 if and only if the label is erroneously classified. By contrast, in the channel learning problem, the noise samples $\left\{z_{i}\right\}_{i=1}^{n}$ are not labeled at all. The learner is required to output a decoder, which is indeed a multiclass classifier (to one of $m$ classes), but is also required to output a codebook $C$. The codebook is an additional requirement from the learner, which is not a part of the multiclass classification problem. Furthermore, even if $C$ is fixed in advance and is not required to be learned, the problem of learning a channel decoder and learning a multiclass classifier are still not equivalent. To see this, note that indeed one can synthesize a multiclass classification problem from $\left\{z_{i}\right\}_{i=1}^{n}$ and a codebook $C$ by considering an augmented data set $\left\{x_{j}+z_{i}\right\}_{j \in[m], i \in[n]}$ of $m n$ data samples. Then, as zero loss is incurred when $x_{j}+z_{i}$ is decoded as $j \in[m]$, one may synthetically attribute the label $j$ to all the points $\left\{x_{j}+z_{i}\right\}_{i \in[n]}$. This indeed results a multiclass classification problem for the labeled data set $\left\{x_{j}+z_{i}, j\right\}_{j \in[m], i \in[n]}$, and the loss function $\ell(C, S, z)$ in 9 is equivalent to the $0-1$ loss function in multiclass classification. However, in general multiclass classification problems, a data set of $m n$ points will not have any specific structure, whereas here it is comprised of $m$ translations of the same $n$ noise samples $\left\{z_{i}\right\}_{i=1}^{n}$. The difference in the structure of the data set then distinguishes between the two problems. Finally, while the decoding regions resulting from the decoding rule (3) are indeed hyperplanes, there is no guarantee that a 
multiclass classifier which separates classes in $\mathbb{R}^{d}$ with hyperplanes can be synthesized as a decoding rule in the form of 3 for some given $S$. This is simply because $S$ is parameterized by at most $O\left(d^{2}\right)$ parameters, whereas a general such classifier is parameterized by $O\left(\mathrm{~m}^{2} d\right)$ such parameters ( $d$ parameters for each hyperplane, and a total of $O\left(\mathrm{~m}^{2}\right)$ hyperplanes, one for each pair of classes). The above differences between the multiclass classification problem and the channel coding problem lead to differences in generalization bounds (c.f. the next Theorem 1 with the generalization bounds for standard multiclass classification [105, Thm. 29.3]).

\section{B. Generalization Error Bounds}

As a cornerstone for learning-theoretic analysis of channel codes, we first establish the following upper bound on the empirical error which is uniform over $(C, S)$ :

Theorem 1. Assume that $n \geq d+1$. With probability of at least $1-\delta$, for all $C \subset\left(\mathbb{R}^{d}\right)^{m}$ with $|C|=m$ and $S \in \mathbb{S}_{+}^{d}$

$$
\left|\mathbf{p}_{\mu}(C, S)-\mathbf{p}_{\boldsymbol{Z}}(C, S)\right| \leq 4 m \sqrt{\frac{2(d+1) \log \left(\frac{e n}{d+1}\right)}{n}}+\sqrt{\frac{2 \log (2 / \delta)}{n}} .
$$

Learning algorithms and ERM: A learning algorithm $A$ : $\left(\mathbb{R}^{d}\right)^{n} \mapsto \mathcal{C} \times \mathcal{S}$ for this problem obtains data samples $Z$ as input and outputs $A_{\boldsymbol{Z}} \equiv\left(C_{\boldsymbol{Z}}, S_{\boldsymbol{Z}}\right)$. Since the right-hand side (r.h.s.) of Theorem 1 uniformly bounds $\mid \mathbf{p}_{\mu}(C, S)-$ $\mathbf{p}_{\boldsymbol{Z}}(C, S) \mid$, it also bounds the generalization error for any learning algorithm. For example, a generic learning algorithm is ERM which chooses $(C, S)_{\text {ERM }}$ that is $\epsilon$-close to $\inf _{C \in \mathcal{C}, S \in \mathcal{S}} \mathbf{p}_{\boldsymbol{Z}}(C, S)$ for some given $\epsilon>0$. The generalization error $\left|\mathbf{p}_{\mu}\left((C, S)_{\mathrm{ERM}}\right)-\mathbf{p}_{\boldsymbol{Z}}\left((C, S)_{\mathrm{ERM}}\right)\right|$ is then bounded by Theorem 1 . Denote by $\left(C_{*}, S_{*}\right)$ a pair which $\epsilon$-achieve $\inf _{C, S} \mathbf{p}_{\mu}(C, S)$. Then, given $n=\tilde{O}\left(\frac{m^{2} d+\log (1 / \delta)}{\epsilon^{2}}\right)$ samples, it holds with probability larger than $1-\delta$ that

$$
\begin{aligned}
\mathbf{p}_{\mu}\left(\left(C_{\boldsymbol{Z}}, S_{\boldsymbol{Z}}\right)_{\mathrm{ERM}}\right) & \leq \mathbf{p}_{\boldsymbol{Z}}\left(\left(C_{\boldsymbol{Z}}, S_{\boldsymbol{Z}}\right)_{\mathrm{ERM}}\right)+\epsilon \\
& \leq \mathbf{p}_{\boldsymbol{Z}}\left(\left(C_{*}, S_{*}\right)\right)+\epsilon \\
& \leq \inf _{C, S} \mathbf{p}_{\boldsymbol{Z}}((C, S))+2 \epsilon \\
& \leq \inf _{C, S} \mathbf{p}_{\mu}(C, S)+3 \epsilon .
\end{aligned}
$$

In general, however, an efficient algorithm to find or approximate the ERM for this problem seems to be out of reach. In the next section, we will demonstrate that one of the reasons for that is that the loss function is discontinuous function of $(C, S)$, and that by properly modifying the loss function, one can propose an algorithm of reasonable complexity.

Proof outline and interpretation of the bound: The proof uses the known relation between uniform error bounds and Rademacher complexity [7] of the loss class induced by the samples and the hypothesis class (codebook and decoder inverse covariance matrix). In accordance, the first term in 11 is an upper bound on the expected Rademacher complexity, while the second term is a high probability bound on the deviation of the expected 
Rademacher complexity from its expected value. This form is similar to other generalization bounds ,e.g, for binary classification [105, Theorem 6.8]. In turn, the expected Rademacher complexity is bounded by analyzing the growth function of the loss class via the Sauer-Shelah lemma, utilizing the fact that the decoding regions are polyhedral, and the separating regions between any pair of codewords is a $d$-dimensional hyperplane, whose Vapnik-Chervonenkis (VC) dimension is bounded by $d+1$. In fact, any other decoding rule for which the pairwise decision rule is chosen from a class of binary classifiers of $\mathrm{VC}$ dimension $d+1$ would lead to the same bound. A common and slightly different approach to bounding the growth function is to define a proper combinatorial dimension which captures the behavior of the growth function of the loss class induced by $\mathbf{p}_{\boldsymbol{Z}}(C, S)$. This can be done via an analogous result to the regular Sauer-Shelah lemma (which is aimed for binary classification and uses the VC dimension). The resulting proof, however, seems to be more complicated than necessary and the result seems to only be (slightly) worse. Nonetheless, this approach could be useful in other scenarios as we discuss below.

Codebook structure: Theorem 1 does not make any assumptions regarding the structure of the codebook, and its generalization bound depends linearly on $m$. We will show in the next section that the modification of the loss function mentioned above also improves the dependency on the number of codewords to nearly square-root. However, if the dimension is high $d \gg 1$, then useful codebooks typically have $m=2^{d R} \gg 1$ codewords (where $R>0$ is the rate per dimension). Therefore, beyond the standard utilization of structured codebooks for efficient encoding and decoding, the learning-based approach also requires to utilize the codebook structure for refined generalization bounds which have better dependency on the number of codewords. Specifically, it is plausible that for some classes of codes, the combinatorial dimension mentioned above could be much lower than its value for general, unstructured, codebooks, and this will lead to generalization bounds which have reasonable dependency on the codebook size.

Learning error exponents: The bound in Theorem 1 represents an additive deviation from the true error probability. However, the required error probability for various communication could be very low, and in these cases the interest is shifted to error exponents, i.e., $\log \mathbf{p}_{\mu}(C, S)$. In these cases, a multiplicative deviation bound is of more importance. However, analysis of the generalization error in this case seems challenging as $\log \mathbf{p}_{\boldsymbol{Z}}(C, S)-$ $\log \mathbf{p}_{\mu}(C, S)$ is not an additive function of the losses over $z_{i}$ (see $(8)$ ), and $\log (\cdot)$ is not a Lipschitz function, so that $\left|\log \mathbf{p}_{\boldsymbol{Z}}(C, S)-\log \mathbf{p}_{\mu}(C, S)\right|$ cannot be directly bounded by $\left|\mathbf{p}_{\boldsymbol{Z}}(C, S)-\mathbf{p}_{\mu}(C, S)\right|$.

The analysis of learning of structured codes and error exponents remains an open problem. The bound in Theorem 1 is comprised of a term related to the complexity of the hypothesis class $\left(\tilde{O}\left(m \sqrt{\frac{d}{n}}\right)\right)$ and a term related only to the required reliability $\left(O\left(\sqrt{\frac{\log (1 / \delta)}{n}}\right)\right)$. We next show that the $O\left(\frac{1}{\sqrt{n}}\right)$ dependency cannot be improved, even for a rather basic setting.

Proposition 2. Let $A_{\boldsymbol{Z}} \equiv\left(C_{\boldsymbol{Z}}, S_{\boldsymbol{Z}}\right)$ be an arbitrary learning algorithm for a codebook of two codewords $m=2$ over $\mathcal{C}=\mathbb{B}^{d}(1)$ and $\mathcal{S}=\left\{S: \zeta_{\min }(S)=1, \zeta_{\max }(S) \leq r_{s}\right\}$ where $r_{s} \leq 2$, where $\zeta_{\max }(S)\left(\right.$ resp. $\left.\zeta_{\min }(S)\right)$ is the 
maximal eigenvalue (resp. minimal) of $S . !^{6}$ Let $\delta \in(0,1 / 4)$ be given. Then,

$$
\sup _{\mu} \mathbb{P}_{\boldsymbol{Z} \sim \mu^{\otimes n}}\left[\mathbf{p}_{\mu}\left(C_{\boldsymbol{Z}}, S_{\boldsymbol{Z}}\right)-\inf _{C, S} \mathbf{p}_{\mu}(C, S)>\frac{27}{100 \cdot r_{s}} \cdot \sqrt{\frac{1}{n} \log \left(\frac{1}{4 \delta}\right)}\right] \geq \delta .
$$

Proof outline: The proof such results is typically based on a reduction to binary hypothesis testing, obtained by establishing that if a learning algorithm does not implicitly distinguish between the true distribution and an alternative distribution then the resulting loss is large (even though such identification is not a requirement from the learner). In standard classification [105, Sec. 28.2] and estimation [112] problems, one postulates a prior probability on the two distributions, and then the specific structure of the loss function allows to show that the optimal learning algorithm should operate as if the true distribution is known to be the one more likely out of the two, given the noise samples. However, in the channel code learning problem, given a posterior distribution on a pair of noise distributions, the optimal codebook/covariance matrix pair may not match exactly to neither of the distributions, but rather should provide a "compromise" between the two. In the proof of Proposition 2 we use a pair of Gaussian distributions, for which the above phenomenon occurs due to the fact $Q\left(\frac{1}{\sqrt{t}}\right)$ (where $Q(\cdot)$ is the tail distribution function of the standard normal distribution) is neither convex nor concave on $\mathbb{R}_{+}$. To ensure convexity, it is required that the variance of the equivalent noise induced by $C, S$ (which moves the codeword towards the boundary of the two decoding regions) will be bounded by some numerical constant for all $C \in \mathcal{C}, S \in \mathcal{S}$. This explains the necessity of the perhaps unintuitive condition $\zeta_{\max }(S) \leq r_{s}$ in the proposition. If this condition does not hold, that noise variance could be arbitrarily large which invalidates the required condition for convexity (for the sake of intuition, consider the codewords $x_{1}=(1,0)=-x_{2}$ and a decoder which sets the boundary between the decoding regions to be the horizontal axis).

Tightness of the codebook size/dimension in Theorem 1. For classification problems, the tightness of the hypothesis class complexity related term (the first term in the bound of Theorem 11) is established by a proper combinatorial dimension (such as $\mathrm{VC}$ dimension) $D$, and then reducing the learning problem to a hypothesis testing problem involving $2^{D}$ different distributions. Then again, one needs to show that identifying the correct distribution is necessary for learning at sufficiently fast rate (e.g., [105, Lemma 28.1]). When $m>2$, even finding the optimal code for a given noise distribution is difficult, and so using this method to show tightness for the channel codes learning problem seems challenging. Finding alternative methods or showing otherwise that the rate in Theorem 1 is not tight w.r.t. $m$ is left for future research.

\section{LEARning Under a Surrogate to THE ERror Probability Loss Function}

\section{A. Problem Formulation}

We next propose an upper bound to the error probability loss function and consider it to be a surrogate loss function to the error probability loss function. We state a uniform convergence result along with a heuristic alternating-

\footnotetext{
${ }^{6}$ Since both $\mathbf{p}_{\mu}(C, S)$ and $\mathbf{p}_{\boldsymbol{z}}(C, S)$ are insensitive to scaling of $S$, the condition on $S$ implied by $\mathcal{S}$ is equivalent to a condition on the condition number of $S$. The requirement $r_{s} \leq 2$ is merely made in order to simplify the exposition, and any other upper bound on $r_{s}$ would lead to a different constant in the r.h.s. of the inequality defining the event whose probability is bounded below.
} 
minimization algorithm which attempts to minimize this loss function. The starting point is the trivial inequality $\mathbb{1}(t<0) \leq(1-t) \vee 0$, which leads to the following hinge-type upper bound on the error probability loss function

$$
\begin{aligned}
\ell_{j}(C, S, z) & :=\mathbb{1}\left[\min _{j^{\prime} \in[m] \backslash\{j\}}\left(\left\|x_{j}-x_{j^{\prime}}\right\|_{S}^{2}+2\left(x_{j}-x_{j^{\prime}}\right)^{T} S z\right)<0\right] \\
& \leq\left[1-\min _{j^{\prime} \in[m] \backslash\{j\}}\left(\left\|x_{j}-x_{j^{\prime}}\right\|_{S}^{2}+2\left(x_{j}-x_{j^{\prime}}\right)^{T} S z\right)\right] \vee 0 \\
& :=\bar{\ell}_{j}(C, S, z) .
\end{aligned}
$$

Analogously to 8 and 9 , we denote $\bar{\ell}(C, S, z):=\frac{1}{m} \sum_{j=1}^{m} \bar{\ell}_{j}(C, S, z)$, define $\overline{\mathbf{p}}_{Z}(C, S):=\frac{1}{n} \sum_{i=1}^{n} \bar{\ell}\left(C, S, Z_{i}\right)$, as well as $\overline{\mathbf{p}}_{\mu}(C, S)=\mathbb{E}[\bar{\ell}(C, S, Z)]$.

Scaling of inverse covariance matrix: Unlike the error probability loss function, $\bar{\ell}_{j}(C, S, z)$ is not invariant to scaling of $S$, and in fact, the maximal eigenvalue of $S$ (i.e., its operator norm) limits the "resolution" of the decoder to distinguish between two codewords. For illustration, suppose that $S=\sigma \cdot I_{d}$ where $\sigma>0$. We can then consider

$$
\tilde{\ell}_{j, \sigma}(C, z):=\bar{\ell}_{j}\left(C, \sigma I_{d}, z\right)=\left[1-\sigma \cdot \min _{j^{\prime} \in[m] \backslash\{j\}}\left(\left\|x_{j}-x_{j^{\prime}}\right\|^{2}+2\left(x_{j}-x_{j^{\prime}}\right)^{T} z\right)\right] \vee 0
$$

as a family of loss functions indexed by $\sigma$. This loss function can also bound the standard error probability similarly to $(18)$ using the inequality $\mathbb{1}(t<0) \leq(1-\sigma t) \vee 0$ which holds for any $\sigma>0$. The larger $\sigma$ is, the slope of the straight line defining this loss function will be larger, and the surrogate loss function will more severely penalize competing codewords on shorter distances. As this amounts to merely scaling of $S$, we simply set $\sigma=1$, and continue with the definition in (19).

\section{B. A Generalization Error Bound}

The following theorem provides a uniform convergence bound for the surrogate loss function:

Theorem 3. Assume that:

1) $\mathbb{P}_{Z \sim \mu}\left\{Z \in \mathbb{B}^{d}(1)\right\}=1$.

2) $\mathcal{C}=\left(\mathcal{C}_{1}\right)^{m}$ where $\mathcal{C}_{1}=\left\{x \in \mathbb{R}^{d}:\|x\| \leq r_{x}\right\}$ for some $r_{x} \geq 1$.

3) $\mathcal{S}=\left\{S \in \mathbb{S}_{+}^{d}: \zeta_{\max }(S) \leq r_{s}\right\}$ for some $r_{s} \geq 1$.

Then with probability of at least $1-\delta$, for all $C \subset \mathcal{C}$ and $S \in \mathcal{S}$

$$
\left|\overline{\mathbf{p}}_{\mu}(C, S)-\overline{\mathbf{p}}_{\boldsymbol{Z}}(C, S)\right| \leq 112 \cdot \sqrt{\frac{(d \vee m)(d+1) \log \left(31 \cdot d r_{s} r_{x}\right)}{n}}+\sqrt{\frac{2 r^{2} \log (2 / \delta)}{n}},
$$

where $r:=1 \vee 4 r_{x} r_{s}\left(r_{x}+1\right)$.

Empirical risk minimization: Assume the typical case in which $m \geq d$. Repeating $\left[15, n=\tilde{O}\left(\frac{d m+\log (1 / \delta)}{\epsilon^{2}}\right)\right.$ samples suffice to obtain with high probability a $3 \epsilon$-approximation for the minimal error probability using ERM. Specifically, the dependency of the generalization error on the number of codewords has been improved from linear 
in $m$ to nearly square-root. This improved dependency may be attributed to the fact that the loss function $\bar{\ell}(C, S, z)$ is a Lipschitz continuous function of $(C, S)$ (Lemma 14 in Appendix A), and thus "easier" to be minimized compared to the discontinuous $\ell(C, S, z)$. This is reflected in the proof outlined next. We also remark that the proof method used to prove Proposition 2 which shows that $n=\tilde{O}\left(\frac{\log (1 / \delta)}{\epsilon^{2}}\right)$ samples are necessary to learn the regular error probability loss function seems also applicable here.7 The details are omitted.

Proof outline and a comparison to Theorem 17. Similarly to the bound of Theorem 1, the bound (21) on the generalization error is comprised of a term which bounds the expected Rademacher complexity, and a term which is a high probability bound on the deviation of the Rademacher complexity from its expectation. The main goal of the proof is upper bounding the expected Rademacher complexity of the loss class. However, compared to the standard error probability loss function, here the Lipschitzness of the loss function (Lemma 14 in Appendix A-C) makes the Rademacher complexity of the induced loss class a maxima of a sub-Gaussian process. Consequently, bounds on the covering numbers of the loss class can be derived (Lemma 15 in Appendix A-C), which, in turn, are used in Dudley's entropy integral to bound the Rademacher complexity (Lemma 17] in Appendix A-C, see, e.g., [115, Ch. 5] for a discussion on the chaining argument leading to these bounds). By contrast, in Theorem 1, the loss function is not Lipschitz, and this enforces to bound the Rademacher complexity by the growth function. In essence, the growth function is the size of a covering set at scale zero, which is only larger than Dudley's entropy integral, which integrates over covering sizes at increasing scales (which are naturally smaller).

Conditions: The conditions in Theorem 3 are made in order to refrain from over-complicating the analysis. Specifically, analogous conditions to the bounded-noise support and bounded-norm codewords are common in the statistical-learning literature, and were also made in quantizer-learning papers (e.g., [72]). They are mainly assumed because for any bounded interval the function $t \mapsto t^{2}$ is Lipschitz continuous, and this allows the control the supremum of empirical process via contraction methods and concentration inequalities (see a discussion [77, Sec. 1.1]). It is plausible that these conditions can be removed using the techniques of [69], [77], [78].

Numerical constants in (21): In the derivation of the theorem and its proof, a simple form of the numerical constants was favored to tightness, and the conditions $r_{x} \geq 1$ and $r_{s} \geq 1$ are inconsequential and were only made for this purpose. In accordance, the constant in Theorem 3 can be significantly reduced.

\section{Alternating optimization algorithm}

Another benefit of using the surrogate loss function $\bar{\ell}(\cdot)$ is that it allows to introduce a simple alternating optimization algorithm to minimize $\overline{\mathbf{p}}_{\boldsymbol{Z}}(C, S)$, utilizing the fact that the loss function $\bar{\ell}_{j}\left(C, S, z_{i}\right)$ is continuous in $(C, S)$. Nonetheless, $\overline{\mathbf{p}}_{\boldsymbol{Z}}(C, S)$ is not a convex function of $C$ - not even for a fixed $S-$ due to the minimization over $j^{\prime} \in[m] \backslash\{j\}$ appearing in $18, !^{8}$ To circumvent this, we propose the following heuristic. Let us introduce the

\footnotetext{
${ }^{7}$ Namely, lower bounding the loss by taking the worst case of the same pair of noise distributions defined in the proof, but modifying Lemma 10 to match the surrogate loss function.

${ }^{8}$ For a fixed $S$, and in case $m=2$, so that there is no need to minimize over $j^{\prime} \in[m] \backslash\{j\}, \overline{\mathbf{p}}_{\boldsymbol{Z}}(C, S)$ is a convex function of $C$ since $\bar{\ell}(C, S, z)$ is a convex function of $C$ by the composition rules of convex/concave functions [14 Ch. 3.2.4]. In general, however, the pointwise minimum of convex functions is not necessarily convex.
} 
auxiliary variables $A:=\left\{\alpha_{j, j^{\prime}}^{(i)}\right\}_{j, j^{\prime} \in[m], i \in[n]}$ where $\alpha_{j, j^{\prime}}^{(i)} \in[0,1]$ and $\alpha_{j, j}^{(i)} \equiv 0$. For any given data sample $z_{i}$ and codeword index $j \in[m]$, we define, with a slight abuse of notation,

$$
\bar{\ell}_{j}\left(C, S, A, z_{i}\right):=\sum_{j^{\prime} \in[m]} \alpha_{j, j^{\prime}}^{(i)}\left[1-\left(\left\|x_{j}-x_{j^{\prime}}\right\|_{S}^{2}+2\left(x_{j}-x_{j^{\prime}}\right)^{T} S z_{i}\right)\right]
$$

and would ideally like to set $A$ such that $\bar{\ell}_{j}\left(C, S, A, z_{i}\right)=\bar{\ell}_{j}\left(C, S, z_{i}\right)$. To wit, if the clipping operation is active and $\bar{\ell}_{j}\left(C, S, z_{i}\right)=0$ then $\alpha_{j, j^{\prime}}^{(i)}=0$ for all $j^{\prime}$. Otherwise, $\alpha_{j, j^{\prime}}^{(i)}$ is arbitrarily supported on

$$
j^{\prime} \in \underset{j^{\prime} \in[m] \backslash\{j\}}{\arg \min }\left(\left\|x_{j}-x_{j^{\prime}}\right\|_{S}^{2}+2\left(x_{j}-x_{j^{\prime}}\right)^{T} S z_{i}\right)
$$

(for example, uniformly), and satisfies $\sum_{j, j^{\prime} \in[m]} \alpha_{j, j^{\prime}}^{(i)}=1$. Clearly, $\left\{\alpha_{j, j^{\prime}}^{(i)}\right\}_{j^{\prime} \in[m]}$ depend on $(C, S)$, and "encode" the nearest neighbors of $x_{j}$ w.r.t. to the noise sample $z_{i}$. The idea of the algorithm is to relax this dependency, and to alternatively update $A$ and $(C, S)$ at each iteration. Given an initial guess $(C, S)$, in the first part of the iteration the value of $A$ is determined, and in the second part of the iteration, given $A$ the value of $(C, S)$ is optimized, or just updated by a stochastic gradient step. Given the new guess for $(C, S)$ the second iteration follows in the same manner, and so on. Let us denote $\bar{\ell}\left(C, S, A, z_{i}\right)=\frac{1}{m} \sum_{j=1}^{m} \bar{\ell}_{j}\left(C, S, A, z_{i}\right)$. For $p \in[m]$, the gradient given the sample $z_{i}$ w.r.t. $x_{p}, p \in[m]$ is given by

$$
\frac{\partial \bar{\ell}\left(C, S, A, z_{i}\right)}{\partial x_{p}}=-\frac{2}{m} \sum_{j \in[m]}\left(\alpha_{j, p}^{(i)}+\alpha_{p, j}^{(i)}\right) S\left(x_{p}-x_{j}\right)+\left(\alpha_{j, p}^{(i)}-\alpha_{p, j}^{(i)}\right) S z_{i},
$$

and w.r.t. $S$ by

$$
\frac{\partial \bar{\ell}\left(C, S, A, z_{i}\right)}{\partial S}=-\frac{1}{m} \sum_{j, j^{\prime} \in[m]} \alpha_{j, j^{\prime}}^{(i)}\left[\left(x_{j}-x_{j^{\prime}}\right)\left(x_{j}-x_{j^{\prime}}\right)^{T}+2\left(x_{j}-x_{j^{\prime}}\right) z_{i}^{T}\right] .
$$

Note that the last gradient may be an asymmetric matrix and does not depend on $S$. The symmetric matrix $\tilde{Q}$ which maximizes $\left\langle\tilde{Q}, \frac{\partial}{\partial S} \bar{\ell}\left(C, S, A, z_{i}\right)\right\rangle$ is clearly given by $\tilde{Q}_{*}=\frac{1}{2} \frac{\partial}{\partial S} \bar{\ell}\left(C, S, A, z_{i}\right)+\frac{1}{2}\left[\frac{\partial}{\partial S} \bar{\ell}\left(C, S, A, z_{i}\right)\right]^{T}$, and so we choose this to be the update direction. The matrix $S$ is then updated to $\hat{S}=S-\eta \tilde{Q}_{*}$ where $\eta>0$ is a step size. This is a symmetric matrix and can be decomposed as $\hat{S}=\sum_{q=1}^{d} \zeta_{q} v_{q} v_{q}^{T}$ where $\left\{\zeta_{q}\right\}$ are the eigenvalues and $v_{q}$ are the eigenvectors. We then project $\hat{S}$ to $\tilde{S} \in \mathcal{S} \subset \mathbb{S}_{+}^{d}$ as

$$
\tilde{S}=\frac{1}{\frac{r_{s}}{\zeta \max } \wedge 1} \sum_{q=1}^{d}\left(\mu_{q} \vee 0\right) \cdot v_{q} v_{q}^{T}
$$

which makes sure that $\tilde{S}$ is a nonnegative definite matrix and that its maximal eigenvalue is less than $r_{s}$.

Algorithm 1 lists a possible SGD variant of such algorithm. A SGD algorithm is specifically adequate for the alternating optimization since a small change in $(C, S)$ is not expected to change most of the $\left\{\alpha_{j, j^{\prime}}^{(i)}\right\}$. The inputs to the algorithm are the noise samples $\boldsymbol{z}$, initial values for $(C, S)$, and step sizes for their update. For each $z_{i}$, first the values of $\left\{\alpha_{j, j^{\prime}}^{(i)}\right\}_{j, j^{\prime} \in[m]}$ are determined according to the current codebook $C^{(i-1)}=\left\{x_{j}^{(i-1)}\right\}_{j \in[m]}$, and second, 
they are updated using the gradients in (24) and (25) (computed based on the single sample $z_{i}$ ).

Convergence analysis: A different version of Algorithm 1 1 of higher computational complexity is a gradient descent algorithm in which the gradient is averaged over all $n$ samples at each iteration. The resulting algorithm is then an iterative generalized alternating optimization algorithm [45], which is a descent algorithm for the empirical surrogate loss $\overline{\mathbf{p}}_{\boldsymbol{Z}}(C, S)$. While it is guaranteed that the loss decreases at each iteration, it is not a priori clear that such algorithm globally converges in the (rather weak) sense that the iterations $\left\{C^{(i)}, S^{(i)}\right\}_{i=1}^{\infty}$ tend to a limit for any given initialization [130]. Furthermore, it is not a priori clear that the sequence of algorithms which are based on increasing number of samples is consistent in the sense that such algorithm tends as $n \rightarrow \infty$ to the ideal population version of this algorithm, which is based on the true distribution of the noise $\mu$ (in a manner that can be made precise, see [100]). Nonetheless, properties of this nature were proved for canonical alternating-minimization algorithms like the expectation-maximization (EM) algorithm [125] and its variants [45], the Lloyd-Max ( $k$-means) algorithm [100]. In addition, such properties were recently proved [124] for a quantizer design problem which utilizes a surrogate loss function similar in spirit to the one studied here. As the analysis there is of technical nature and it seems that similar ideas can be applied to this paper we do not pursue this direction here. Anyhow, if one has obtained $\overline{\mathbf{p}}_{\boldsymbol{Z}}\left(C^{\left(i_{*}\right)}, S^{\left(i_{*}\right)}\right)$ below an acceptable threshold for some $i_{*} \in \mathbb{N}$ using Algorithm 1 , then the expected error probability $\overline{\mathbf{p}}_{\mu}\left(C^{\left(i_{*}\right)}, S^{\left(i_{*}\right)}\right)$ is bounded as in Theorem 3 .

\section{LEARNING BY CODEBOOK EXPURGATION}

\section{A. Problem Formulation and the Gibbs Algorithm}

In general, finding an optimal codebook is a difficult task even when the noise distribution $\mu$ is known. A simple way to approach this problem is to select the $m$ codewords of the codebook $C$ from a larger super-codebook $C_{0}$ of $m_{0}>m$ codewords. Such a process is amenable to practical implementation, since the super-codebook can be statically chosen in advance, and can be simple or well-structured (such as a grid or a lattice [129]), whereas the $m$ codewords in the codebook can be chosen dynamically based on the noise statistics. This approach is akin to both practical coding methods [36], as well as to the the common technique used in the proofs of random coding bounds on the reliability function of channel coding [38], in which the codebook is expurgated from codewords of large conditional error probability. We assume in this section, that the decoder inverse covariance matrix is fixed (say $S=I_{d}$ ), and thus omit $S$ from the notation.

Nonetheless, even when $\mu$ is known, finding the optimal set of $m$ codewords is a combinatorial optimization problem, which is computationally heavy when $m_{0} \gg m$. To see this, consider the simpler combinatorial optimization problem of finding the codebook $C$ which minimizes the average pairwise error probability (known as the union bound estimate)

$$
\underset{C=\left\{x_{1}, \ldots, x_{m}\right\} \subset C_{0}}{\arg \min } \frac{1}{m(m-1)} \sum_{j_{1}, j_{2} \in[m]: j_{1} \neq j_{2}} \mathbf{p}_{\mu}\left(x_{j_{1}} \rightarrow x_{j_{2}}\right),
$$




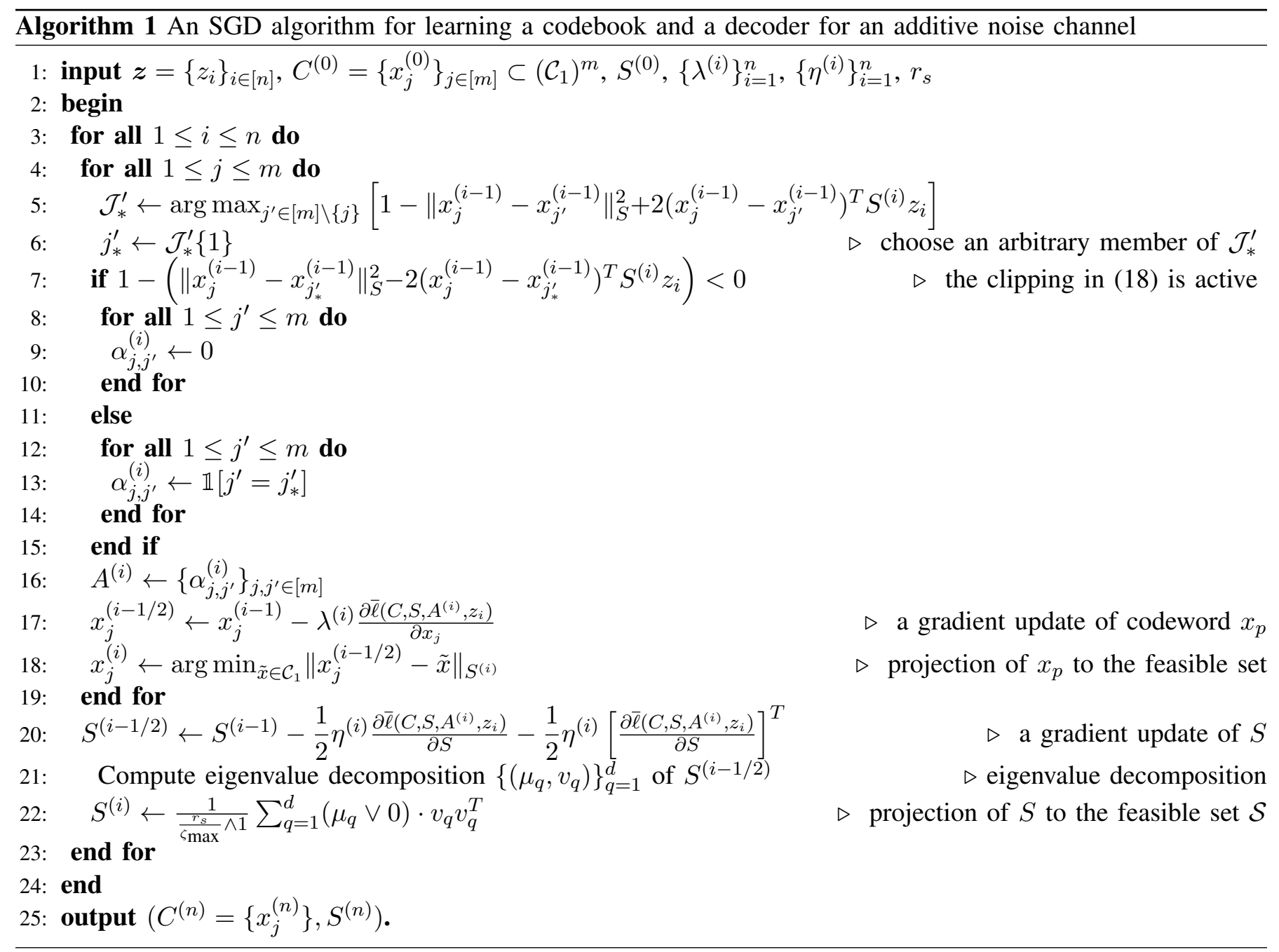

where $\mathbf{p}_{\mu}\left(x_{j_{1}} \rightarrow x_{j_{2}}\right)$ is the error probability of making an error from $x_{j_{1}}$ to $x_{j_{2}}$ when these are the only two codewords in the codebook. The problem [27] is then equivalent to the k-cardinality sub-graph problem [15, Sec. 4] as follows: The $m_{0}$ codewords of $C_{0}$ can be taken as the nodes of a complete directed graph, such that the weight of each edge is $\mathbf{p}_{\mu}\left(x_{j_{1}} \rightarrow x_{j_{2}}\right)$. Then, $\sum_{j_{1}, j_{2} \in[m]: j_{1} \neq j_{2}} \mathbf{p}_{\mu}\left(x_{j_{1}} \rightarrow x_{j_{2}}\right)$ is the total edge weight of the subgraph of cardinality $m$, which only contains the codewords of $C$ as nodes. The problem (27) is then equivalent to finding a sub-graph of cardinality $m$ with minimal weight. Hence, any algorithm which solves or approximates the $k$-cardinality sub-graph problem can be used find the solution to 27). Nonetheless, the $k$-cardinality sub-graph problem is NP-hard [29], and the problem of interest here, of finding

$$
C_{*}=\underset{C=\left\{x_{1}, \ldots, x_{m}\right\} \subset C_{0}}{\arg \min } \mathbf{p}_{\mu}(C)
$$

is only more difficult since the error probability is a more complicated function of the codebook compared to the average pairwise error probability in (27). Similar observations can be made for the empirical error probability.

A possible greedy relaxation to this optimization problem is to approximate the optimum by gradually removing codewords from the codebook, say $k$ of them at each step (as will be evident, $k$ is practically expected to be chosen 
as a small integer). For simplicity of the description we assume henceforth that $T:=\frac{m_{0}-m}{k} \in \mathbb{N}_{+}$. The general meta-algorithm is as follows. Initialize with a codebook $C_{0}$ of $m_{0}$ codewords. Then, for $t=1, \ldots T$ :

1) Construct candidate codebooks $\left\{C_{t}^{[l]}\right\}_{l \in\left[\left(\begin{array}{c}m_{t-1} \\ k\end{array}\right)\right]}$ and evaluate the error probability for each candidate $\mathbf{p}\left(C_{t}^{[l]}\right)$.

2) Choose an index $l^{*}$ and set $C_{t} \equiv\left\{C_{t}^{\left[l_{*}\right]}\right\}$ according to a selection rule (which is based on the the error probabilities). Renumber the codewords in $C_{t}$ by $\left[m_{t}\right]$ where $m_{t}=m_{t-1}-k$.

The error probabilities computed at the first step may be either according to the true distribution $\mu$ or according to the empirical distribution induced by $Z$, and the algorithm is termed, respectively, the population algorithm or empirical algorithm.

A proper choice of a selection rule is a delicate question. For example, a possible variant of such algorithm would remove the $k$ codewords in $C_{t}$ which have the maximal conditional error probability $\mathbf{p}\left(C_{t} \mid j\right)$. However, this is both greedy as well as naive since the decision to remove the codeword $\tilde{x}$ from the codebook should be based on both types of error events - from $\tilde{x}$ when it is the transmitted codeword to a different (competing) codeword, as well as the opposite case in which $\tilde{x}$ is decoded but a different codeword was transmitted. Another problem is that such algorithm depends strongly on the noise samples for the empirical algorithm, and thus might not generalize well to out-of-sample noise.

To circumvent this problem, we next propose a Gibbs algorithm which randomly removes codewords from the codebook. Let $C_{t}$ be a codebook of $m_{t}$ codewords in $\mathcal{C}$, let $Q \in \mathcal{P}\left(\mathbb{R}^{d}\right)$ be a probability reference measure on $\mathbb{R}^{d}$ whose support includes $\mathcal{C}$, and let $\beta>0$ be an inverse temperature parameter. Given $C_{t}$, a Gibbs algorithm chooses to expurgate the codewords with indices $\left\{j_{1}, \ldots j_{k}\right\}$ to obtain $C_{t+1}$ with probability

$$
\mathbb{P}\left[C_{t+1}=C_{t} \backslash\left\{x_{j}\right\}_{j \in\left\{j_{1}, \ldots j_{k}\right\}} \mid \boldsymbol{Z}, C_{t}\right] \propto Q\left(C_{t+1}\right) \cdot \exp \left[-\beta \cdot \mathbf{p}_{\boldsymbol{Z}}\left(C_{t+1}\right)\right]
$$

For $0<\beta<\infty$, the algorithm compromises between the two extremes of removing codewords at random according to the prior distribution $Q(\beta \rightarrow 0)$ versus strong dependence on the noise samples $(\beta \rightarrow \infty)$. The Gibbs algorithm is listed in Algorithm 2

Computational load: Note that the computation of $\mathbf{p}_{\boldsymbol{Z}}\left(C_{t+1}\right)$ requires not only removing the $\mathbf{p}_{\boldsymbol{Z}}\left(C_{t} \mid j_{p}\right)$, $p \in[k]$ from the averaging operation in the error probability, but should also take into account that the decoder cannot err to the codewords $\left\{x_{j}\right\}_{j \in\left\{j_{1}, \ldots j_{k}\right\}}$. This has to be done for each of the $\left(\begin{array}{c}m_{t-1} \\ k\end{array}\right)$ candidate codebooks, and so the choice of $k$ significantly determines the complexity via the required number of candidate codebooks, where the latter is upper bounded by $m_{0}^{k}$ (see further discussion on efficient implementation in Section VI-B. However, once the algorithm's parameters are fixed, so is the running time of the algorithm. Furthermore, the algorithm actually produces codebooks of any size $m \leq m^{\prime} \leq m_{0}$, typically with lower error probability for smaller codebook, and thus the codebook size can be dynamically chosen. We also remark that after being learned by the decoder, the chosen codebook can be sent back to the encoder via a feedback link using no more than $m \log _{2} m_{0}$ bits.

To analyze the error of the algorithm, we arbitrarily set $C_{0}$, and let $C_{\mu}=\left(C_{0}, C_{\mu, 1}, \ldots, C_{\mu, T}\right)$ (resp. $C_{\boldsymbol{Z}}=$ 


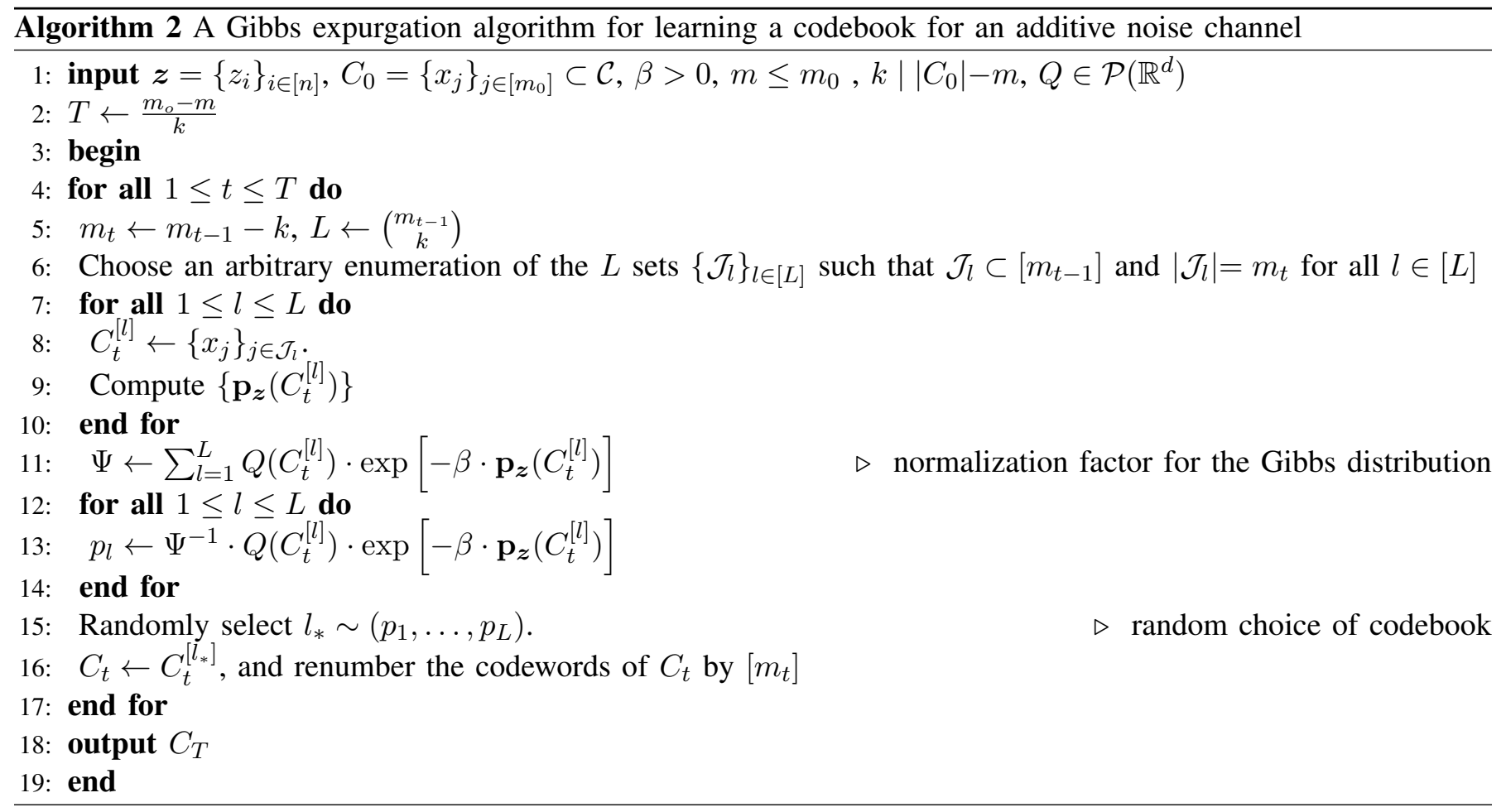

$\left.\left(C_{0}, C_{\boldsymbol{Z}, 1}, \ldots, C_{\boldsymbol{Z}, T}\right)\right)$ be the sequence of random codebooks generated by the population (resp. empirical) Gibbs algorithm, when both are initialized with $C_{0}$. Let $C_{*} \subset C_{0}$ be the codebook obtained by optimal expurgation, as in (28). The average excess error probability of the empirical Gibbs algorithm can be decomposed as

$$
\mathbb{E}\left[\mathbf{p}_{\mu}\left(C_{\boldsymbol{Z}, T}\right)-\mathbf{p}_{\mu}\left(C_{*}\right)\right]=\underbrace{\mathbb{E}\left[\mathbf{p}_{\mu}\left(C_{\boldsymbol{Z}, T}\right)-\mathbf{p}_{\mu}\left(C_{\mu, T}\right)\right]}_{\text {empirical error }}+\underbrace{\mathbb{E}\left[\mathbf{p}_{\mu}\left(C_{\mu, T}\right)-\mathbf{p}_{\mu}\left(C_{*}\right)\right]}_{\text {approximation error }}
$$

where the expectations are taken w.r.t. both the randomness of $\boldsymbol{Z}$ and the Gibbs algorithm. The empirical error is a result of using the empirical distribution of $Z$ in lieu of the true distribution $\mu$ in the Gibbs algorithm, and as we shall see is upper bounded by $\tilde{O}\left(\beta \sqrt{\frac{T}{n}}\right)$, and vanishes as $n \rightarrow \infty$. By contrast, the approximation error seems to be an inevitable price to pay for using using a computationally feasible method (Gibbs algorithm) for (approximately) solving (28). It cannot be reduced by increasing the number of samples, and seems challenging to quantify due to its intricate dependency on the noise distribution.

An alternative algorithm: As said, finding $C_{*}$ which minimizes $\mathbf{p}_{\mu}(C)$ in 28 is a combinatorial optimization problem, and as such it can be tackled using the simulated annealing approach [62]. This approach is based on local optimization of the codebook. In its naive form, such algorithm is initialized with a the super-codebook $C_{0}$ of $m_{0}$ codewords, and a codebook $C^{(1)} \subset C_{0}$ of $m$ codewords. At iteration $t \in \mathbb{N}_{+}$, the codebook $C^{(t+1)} \subset C_{0}$ is chosen as the codebook which minimizes the error probability among all codebooks of size $m$ which are different from $C^{(t)}$ by $k$ codewords, if such exists, and otherwise the algorithm stops and outputs its current codebook. As well known, the simulated annealing [59] replaces the "hill-descending" step, with a randomized step which though it might increase the objective function (error probability), is essential in order to avoid local minima. The same 
algorithm can be used to minimize $\mathbf{p}_{\boldsymbol{Z}}(C)$ in the empirical setting, however, as discussed above, this might not guarantee generalization to out-of-sample noise. Nonetheless, it is easy to see that generalization bounds similar to Theorems 4 and 5 can be obtained for an algorithm which chooses $C$ randomly from

$$
\mathbb{P}[C \mid \boldsymbol{Z}] \propto Q(C) \cdot \exp \left[-\beta \cdot \mathbf{p}_{\boldsymbol{Z}}(C)\right] \cdot \mathbb{1}\left[C \subset C_{0}:|C|=m\right]
$$

Sampling directly from 31 is difficult (since this distribution is supported on $\left(\begin{array}{c}m_{0} \\ m\end{array}\right)$ different codebooks and so it is costly or impossible to compute the required normalization factor), however it can be done indirectly via the Metropolis-Hastings algorithm [11], [50], [84], which generates a Markov chain whose stationary distribution approaches that of (31). Nonetheless, the time required to converge to 31) (mixing time) might be large. Analysis of this method, and experiments which compare its effectiveness with that of Algorithm 2 are left for future research.

\section{B. Generalization Error and Empirical Error Bounds}

The next theorem states a bound on the average empirical error, and also provides a bound on the average generalization error $\mathbb{E}\left[\mathbf{p}_{\mu}\left(C_{\boldsymbol{Z}, T}\right)-\mathbf{p}_{\boldsymbol{Z}}\left(C_{\boldsymbol{Z}, T}\right)\right]$.

Theorem 4. Assume that $C_{0}$ of size $\left|C_{0}\right|$ is chosen in a data-independent way, that the Gibbs algorithm is used with $T=\frac{m_{0}-m}{k} \in \mathbb{N}_{+}$steps, and inverse-temperature $\beta>0$. Also assume that $m_{0} \geq 2 m$ and

$$
\beta^{2}\left(\log n+\left(\frac{m_{0}}{2}+1\right) \log m_{0}-\log k\right) \leq \frac{n}{2},
$$

Then, the average empirical error is bounded as

$$
\mathbb{E}\left[\mathbf{p}_{\mu}\left(C_{\boldsymbol{Z}, T}\right)-\mathbf{p}_{\mu}\left(C_{\mu, T}\right)\right] \leq 3 \sqrt{\frac{T \beta^{2}\left(\log n+m_{0} \log m_{0}-\log k\right)}{n}}
$$

and the average generalization error is upper bounded as

$$
\mathbb{E}\left[\mathbf{p}_{\mu}\left(C_{\boldsymbol{Z}, T}\right)-\mathbf{p}_{\boldsymbol{Z}}\left(C_{\boldsymbol{Z}, T}\right)\right] \leq \sqrt{T\left(\frac{\beta}{n} \wedge \frac{\beta^{2}}{4 n^{2}}\right)} .
$$

Discussion: The average empirical error bound is on the order of $O\left(\sqrt{\frac{T \beta^{2} \log n}{n}}\right)$. The proof is based on showing that $\left|\mathbf{p}_{\boldsymbol{z}}(C)-\mathbf{p}_{\mu}(C)\right| \leq \epsilon$ for all possible sub-codebooks $C \subset C_{0}$ with high probability (the $\sqrt{\log n}$ dependency can be traced in the proof to a union bound which leads to this result), and using this to bound generalization error by bounding the KL divergence $\mathrm{d}_{\mathrm{KL}}\left(P_{C_{\boldsymbol{z}, 1} \cdots C_{\boldsymbol{z}, T}} \| P_{C_{\mu, 1} \cdots C_{\mu, T}}\right)$ where $P_{C_{\mu, 1} \cdots C_{\mu, T}}$ (resp. $\left.P_{C_{z, 1} \cdots C_{\boldsymbol{z}, T}}\right)$ is the the joint probability measure of $\boldsymbol{C}_{\mu}$ (resp. $\boldsymbol{C}_{\boldsymbol{Z}}$ ). The average generalization error is on the order of $O\left(\sqrt{T\left(\frac{\beta}{n} \wedge \frac{\beta^{2}}{n^{2}}\right)}\right)$, which, in the standard case $\beta<n$ is $O\left(\sqrt{T} \frac{\beta}{n}\right)$, which decays faster than the average empirical error bound. The bound is proved by establishing an information-theoretic stability of the Gibbs algorithm, and using the results of [128] which bounds the generalization error of stable algorithms.

The condition $m_{0} \geq 2 m$ is only made for simplicity of exposition of the proof. The generalization error can be used to estimate a bound on $\mathbf{p}_{\mu}\left(C_{\boldsymbol{Z}, T}\right)$ using $\mathbf{p}_{\boldsymbol{Z}}\left(C_{\boldsymbol{Z}, T}\right)$, which in turn can be computed from the data $\boldsymbol{Z}$. It 
should be noted, however, that unlike Theorems 1 and 3 , the stated bound is on the average error, and has no strong concentration properties. This is a result of the proof method which relies on the chain rule properties of the KL divergence (and mutual information) to bound the average error. A bound which holds with high probability will be presented in what follows. Evidently, both upper bounds in Theorem 4 are monotonically increasing functions of $\beta$, and thus $\beta$ should be as low as possible in order to minimize the bounds on the empirical and generalization errors. This can be contrasted with the goal of minimizing $\mathbf{p}_{\mu}\left(C_{\mu, T}\right)$ (or $\mathbf{p}_{\boldsymbol{Z}}\left(C_{\boldsymbol{Z}, T}\right)$ ) which typically requires $\beta$ to be as "large" as possible.

Assuming that $\frac{\beta}{n} \rightarrow 0$ as $n \rightarrow \infty$, the average generalization error indicated by Theorem 4 is $O\left(\frac{\sqrt{T} \beta}{n}\right)$. This bound involves averaging on both the randomness of the data samples $\boldsymbol{Z}$, as well as the randomness of the Gibbs algorithm. To obtain high probability bounds, we rely on a uniform-stability property of the Gibbs algorithm.9 Specifically, if we assume the stronger condition that $\frac{T \beta}{n} \rightarrow 0$ as $n \rightarrow \infty$ then a high probability of roughly the same order can also be obtained. Specifically, for a given algorithm $P_{C \mid Z, C_{0}}$, and a single noise sample $\tilde{z} \in \mathbb{R}^{d}$ we let

$$
\mathbf{q}_{\tilde{z}}(\boldsymbol{z}):=\mathbb{E}\left[\mathbf{p}_{\tilde{z}}(C) \mid \boldsymbol{Z}=\boldsymbol{z}, C_{0}\right]
$$

be the error probability of $\tilde{z}$ when averaged over a random codebook $C$ that is drawn according to $P_{C \mid \boldsymbol{Z}=\boldsymbol{z}, C_{0}}$ (defined via the Gibbs algorithm), and $\mathbf{q}_{\mu}(\boldsymbol{z}):=\mathbb{E}_{\tilde{Z} \sim \mu}\left[\mathbf{q}_{\tilde{Z}}(\boldsymbol{z})\right]$ as well as $\mathbf{q}_{\tilde{\boldsymbol{Z}}}(\boldsymbol{z}):=\frac{1}{n} \sum_{i=1}^{n} \mathbf{q}_{\tilde{z}_{i}}(\boldsymbol{z})$. We have the following:

Theorem 5. Assume that $\frac{T \beta}{n} \rightarrow 0$ as $n \rightarrow \infty$. Then, there exists an absolute $n_{0} \in \mathbb{N}_{+}$and an absolute constant $c>0$ such that for all $n \geq n_{0}$

$$
\mathbb{P}\left[\mathbf{q}_{\mu}(\boldsymbol{Z})-\mathbf{q}_{\boldsymbol{Z}}(\boldsymbol{Z})>c\left(\frac{\sqrt{T} \beta}{n}+\frac{1}{\sqrt{n}}\right) \cdot \log \left(\frac{n}{\beta \sqrt{T}}\right) \cdot \log n \cdot \log \frac{n}{\delta}\right] \leq \delta .
$$

Hence, under the condition of Theorem 5, and assuming that $\beta=\Omega\left(\sqrt{\frac{n}{T}}\right)$, the high probability bound is $\tilde{O}\left(\frac{\sqrt{T} \beta}{n}\right)$, and matches, up to logarithmic factors, the average generalization error bound of Theorem 4

Discussion: As discussed, the output of the Gibbs algorithm is random due to both the randomness of the samples and the randomness of the Gibbs mechanism. While the generalization bound of Theorem 4 is averaged w.r.t. both type of randomness, the bound of Theorem 5 is a high probability bound w.r.t. the samples, but still averages the Gibbs mechanism. Nonetheless, assuming $\frac{T \beta}{n}=o(1)$ the decay rate of Theorem 4 is recovered (up to logarithmic terms), and the generalization error is $\tilde{O}\left(\frac{\sqrt{T} \beta}{n}\right)$ with high probability, and not only on the average. The bound 36 is proved by establishing that the Gibbs algorithm is a differentially-private algorithm [27] (Lemma 19 in Appendix A-D), which, in turn, implies that it is uniformly stable learning algorithm [13]. Then, the recent high probability bound on the generalization error of uniformly stable learning algorithms [34] is utilized.

\footnotetext{
${ }^{9}$ Recently, 30 proposed to use the Rényi mutual information between the data samples $\boldsymbol{Z}$ and the algorithm output, but the resulting bounds in this setting are weaker than the ones stated here.
} 


\section{LeARning InPut Distributions which Maximize Mutual Information}

\section{A. Problem Formulation}

Communicating at rates which approach the capacity of the additive noise channel (1) requires knowledge of the noise distribution $\mu_{Z}{ }^{10}$ in order to optimize the input distribution which we denote here by $\mu_{X}{ }^{11}$ Thus, whenever coding across multiple $d$-dimensional codewords is possible, it is desired to find the input distribution $\mu_{X}$ which maximizes the mutual information between the input and the output, or, equivalently, the differential entropy of the channel output. Typically, to obtain finite mutual information, the set of feasible input distributions is restricted to some $\mathcal{P}$ and so it is required to solve:

$$
\underset{\mu_{X} \in \mathcal{P}}{\arg \max } I(X ; X+Z)=\underset{\mu_{X} \in \mathcal{P}}{\arg \max } h(X+Z)
$$

where we assume that the maximum exists (otherwise, it is required to find an $\epsilon>0$ approximation of the supremum).

Closed-form solutions to (37) are rare, and currently exist only for the simplest classes $\mathcal{P}$, even when the noise distribution is completely known. For example, for a known Gaussian noise $Z \sim N\left(0, I_{d}\right)$, the optimal input distribution is Gaussian if $\mathcal{P}$ represents an average power constraint, but if an amplitude constraint is also enforced, to wit

$$
\mathcal{P}=\left\{\mu_{X}: \mathbb{P}_{\mu_{X}}\left(\|X\| \leq A_{X}\right)=1, \mathbb{E}_{\mu_{X}}\|X\| \leq d \sigma_{X}^{2}\right\}
$$

then it is only known that the optimal $\mu_{X}$ is supported on a finite number of concentric shells with isotropic direction (see [98], [106], [107], and [28] for an overview and recent advances). Finding the optimal support, however, is not trivial, and requires algorithmic efforts, e.g., the cutting-plane iterative algorithm proposed in [53] for finding a discrete approximation to the capacity input distribution. Similarly, for the class of input distributions which are restricted to some $m$-point codebook $C \subset \mathbb{R}^{d}$ whose power is bounded, to wit,

$$
\mathcal{P}=\left\{\mu_{X}: \mathbb{E}_{\mu_{X}}\|X\| \leq d \sigma_{X}^{2},\left|\operatorname{supp}\left(\mu_{X}\right)\right| \leq m\right\}
$$

it is only known that for $d=1$ the optimal input distribution weakly converges to an equi-lattice when $\sigma_{X} \rightarrow \infty$ and to a Gaussian quadrature when $\sigma_{X} \rightarrow 0$ [89], [126, Sec. IV]. Nonetheless, from an algorithmic point of view, if the support is restricted to a fixed codebook $C=\left\{x_{j}\right\}_{j \in[m]} \subset \mathbb{R}^{d}$ and

$$
\mathcal{P}=\left\{\mu_{X}=\sum_{j=1}^{m} a_{j} \delta_{x_{j}}: \mathbb{E}_{\mu_{X}}\|X\| \leq d \sigma_{X}^{2}, x_{j} \in \mathbb{R} \forall j \in[m], \boldsymbol{a} \in \mathbb{A}^{m-1}\right\},
$$

\footnotetext{
${ }^{10}$ In this section we add the sub-script $Z$ to $\mu$ and explicitly $\mu_{Z}$, so it will not be confused with the input distribution $\mu_{X}$.

${ }^{11}$ As was considered in previous sections, the design of the decoder is also based on the noise distribution, and in uncoded systems will affect the error probability. However, in coded systems, and especially in the random coding regime, this can be circumvented in principle by use of universal decoders which also achieve capacity [65].
} 
where $\delta_{x_{0}}=\delta\left(x-x_{0}\right)$ and $\delta(x)$ is Dirac's delta function, then the problem of maximizing $h(X+Z)$ over the weights $\boldsymbol{a}$ (in a feasible subset of $\mathbb{A}^{m-1}$ dictated by the power constraint) is a convex optimization problem and can be solved using the celebrated Blahut-Arimoto algorithm [3], [9].

Following the approach previously taken in this paper, we focus on the statistical-learning aspect of this problem, i.e., the difference between empirical and population versions of this optimization problem, when the noise distribution is unknown, and instead $n$ i.i.d. samples of the noise are available. We consider two classes for input distributions, and show for each one that an estimator $\hat{h}_{Z}(X+Z)$ to $h(X+Z)$ which is based on the noise samples converges with high probability to the true value and that the convergence is uniform over the chosen class $\mathcal{P}$. As in the previous sections, this assures that any algorithm which attempts to maximize $\hat{h}_{\boldsymbol{Z}}(X+Z)$ (that can be computed from data) will produce $h(X+Z)$ which is not very far from the computed value, where specific convergence rates depend on the class of input distributions.

\section{B. A General Class of Input Distributions}

The first class of input distributions we consider is rather general, but in turn the resulting convergence rate assured is only $\tilde{O}\left(n^{-1 / d}\right)$ (Theorem 6), and as such deteriorates fast with the dimension $d$. Following the above discussion, there is also no known efficient algorithm that finds the maximizing distribution in this class even for a known noise distribution. Nonetheless, the motivation of deriving such a result is to demonstrate that uniform convergence is a possible even for quite general classes. The results itself necessitates further discussion, and this follows its formal statement.

Following [92], we say that a density $\mu_{X}$ (absolutely continuous w.r.t. Lebesgue measure) is $\left(\psi_{1}, \psi_{2}\right)$-regular for $\psi_{1}>0$ and $\psi_{2} \geq 0$ if

$$
\left\|\nabla \log \mu_{X}(x)\right\| \leq \psi_{1}\|x\|+\psi_{2}, \forall x \in \operatorname{supp}\left(\mu_{X}\right) .
$$

We then consider input densities $\mu_{X}$ which are $\left(\psi_{1}, \psi_{2}\right)$-regular, have a bounded second moment, and have a absolutely bounded entropy:

$$
\mathcal{P}^{*}\left(\eta_{X}, \sigma_{X}, \psi_{1}, \psi_{2}\right):=\left\{\mu_{X} \text { is }\left(\psi_{1}, \psi_{2}\right) \text {-regular: } \mathbb{E}_{\mu_{X}}\|X\|^{2} \leq d \sigma_{X}^{2},\left|h\left(\mu_{X}\right)\right| \leq \eta_{X}\right\}
$$

Theorem 6. Assume that $\mu_{Z}$ is a probability density on $\mathbb{R}^{d}$ such that $A:=\|Z\|$ is $\left(\sqrt{d} \sigma_{Z}\right)$-sub-Gaussian where $\sigma_{Z}=\Omega\left(n^{-(d-2) /(4 d)}\right)$. Let $\boldsymbol{Z}=\left(Z_{1}, \ldots, Z_{n}\right) \stackrel{\text { i.i.d. }}{\sim} \mu_{Z}$, and let $\hat{Z}_{n} \sim \frac{1}{n} \sum_{i=1}^{n} \delta_{Z_{i}}$ denote the empirical distribution of $\boldsymbol{Z}$. Then, there exists $n_{0} \in \mathbb{N}_{+}$and a constant $c>0$, both which depend on $\left(d, \sigma_{X}, \eta_{X}, \psi_{1}, \psi_{2}, \sigma_{Z}\right)$ such that for all $n \geq n_{0}$

$$
\max _{X \sim \mu_{X}: \mu_{X} \in \mathcal{P}^{*}\left(\eta_{X}, \sigma_{X}, \psi_{1}, \psi_{2}\right)}\left|h(X+Z)-h\left(X+\hat{Z}_{n}\right)\right| \leq c \frac{\log ^{2} n}{n^{1 /(d \vee 4)}}
$$

with probability larger then $1-\frac{1}{n}$.

We proceed with the following discussion. 
Validity of the estimator: The estimator $h\left(X+\hat{Z}_{n}\right)$ in 43 is indeed well-defined. This is because whenever $X$ has a density $\mu_{X}$, so does $X+\hat{Z}_{n} \sim \frac{1}{n} \sum_{i=1}^{n} \mu_{X} * \delta_{Z_{i}}$ which is a mixture of $n$ translations of $\mu_{X}$.

Conditions on the noise distribution: The assumption $\sigma_{Z}=\Omega\left(n^{-(d-2) /(4 d)}\right)$ is rather mild, and made to simplify the resulting bound (see Lemma 21).

Conditions on the input distribution: The regularity condition (41) defines a smoothness condition on $\mu_{X} \in \mathcal{P}^{*}$, and implies, for example, that the tail of the density cannot decay faster than the tail of a Gaussian density, because regularity implies

$$
\mu_{X}(x) \geq \exp \left[-\left|\log \mu_{X}(0)\right|-\frac{\psi_{1}}{2}\|x\|^{2}-\psi_{2}\|x\|\right]
$$

The finite entropy requirement in $\mathcal{P}^{*}$ can be assured by controlling $\left|\log \mu_{X}(0)\right|$, since if $\mu_{X}$ is $\left(\psi_{1}, \psi_{2}\right)$-regular and $\mathbb{E}\|X\|^{2} \leq d \sigma_{X}^{2}$ then $[92$, Sec. II]

$$
\left|h\left(\mu_{X}\right)\right| \leq\left|\log \mu_{X}(0)\right|+\psi_{2} \sqrt{d} \sigma_{X}+\frac{\psi_{1}}{2} d \sigma_{X}^{2},
$$

and this upper bound can be chosen as $\eta_{X}$.

Non-density input classes: Suppose that $\tilde{\mathcal{P}}:=\left\{\tilde{\mu}_{X}: \mathbb{E}\|X\|^{2} \leq d \sigma_{X}^{2}\right\}$ is a set of probability measures (which are not necessarily densities w.r.t. the Lebesgue measure). Now consider the set of smoothed densities

$$
\overline{\mathcal{P}}:=\left\{\tilde{\mu}_{X} * \varphi_{0, \sigma}: \tilde{\mu}_{X} \in \tilde{\mathcal{P}}\right\}
$$

where $\varphi_{\eta, \sigma}$ is the Gaussian density with mean $\eta \in \mathbb{R}^{d}$ and covariance matrix $d \sigma^{2} \cdot I_{d}$. It was shown recently in [40] that estimating the entropy of a smoothed distribution can be made at a fast rate of $e^{O(d)} \cdot O\left(\frac{1}{\sqrt{n}}\right)$. The smoothness operation is also useful here, though not for improving the error rates but rather to allow for general input distributions. By [92, Prop. 2], any $\bar{\mu}_{X} \in \overline{\mathcal{P}}$ is $\left(\bar{\psi}_{1}, \bar{\psi}_{2}\right):=\frac{\log e}{d \sigma^{2}}\left(3,4 \sqrt{d} \sigma_{X}\right)$-regular. Furthermore, $\left|h\left(\bar{\mu}_{X}\right)\right|$ is bounded because for $\bar{\mu}_{X}=\tilde{\mu}_{X} * \varphi_{0, \sigma}$ and $\tilde{X} \sim \tilde{\mu}_{X}, \tilde{X} \Perp W \sim \varphi_{0, \sigma}$

$$
h\left(\bar{\mu}_{X}\right)=h(\tilde{X}+W) \geq h(W)=\frac{d}{2} \log \left(2 \pi e \sigma^{2}\right):=\bar{\eta}_{X}^{(-)},
$$

and since Gaussian vector maximizes entropy under a variance constraint

$$
h\left(\bar{\mu}_{X}\right)=h(\tilde{X}+W) \leq \frac{d}{2} \log \left(2 \pi e\left(\sigma^{2}+\sigma_{X}^{2}\right)\right):=\bar{\eta}_{X}^{(+)} .
$$

Thus, any $\bar{\mu}_{X} \in \mathcal{P}^{*}\left(\bar{\eta}_{X}, \bar{\sigma}_{X}, \bar{\psi}_{1}, \bar{\psi}_{2}\right)$ where $\bar{\eta}_{X}=\left|\bar{\eta}_{X}^{(-)}\right| \vee\left|\bar{\eta}_{X}^{(+)}\right|$and $\bar{\sigma}_{X}:=d\left(\sigma^{2}+\sigma_{X}^{2}\right)$, and the result of Theorem 6 holds for the smoothed class of input densities $\overline{\mathcal{P}}$. Nonetheless, it seems difficult to make any claims regarding the loss in mutual information due to the Gaussian smoothing operation (and this is actually the motivation for the restriction to regular densities in Theorem 6 to begin with).

Proof idea: The absolute difference in differential entropy of a pair of regular densities can be controlled by the second-order Wasserstein distance [92, Sec. II]. Specifically, assume that both $X+Z$ and $X+\hat{Z}_{n}$ are 
$\left(\psi_{1}, \psi_{2}\right)$-regular densities, then [92, Prop. 1]

$$
\left|h(X+Z)-h\left(X+\hat{Z}_{n}\right)\right| \leq\left(\frac{\psi_{1}}{2} \sqrt{\mathbb{E}\|X+Z\|^{2}}+\frac{\psi_{1}}{2} \sqrt{\mathbb{E}\left\|X+\hat{Z}_{n}\right\|^{2}}+\psi_{2}\right) \cdot W_{2}\left(\mu_{X+Z}, \mu_{X+\hat{Z}_{n}}\right) .
$$

As discussed in [92], this bound can be considered a reversed version of transportation-information inequalities [12], [74], [96] which upper bound the Wasserstein distance by the KL divergence (where the latter is related to entropy difference). Anyway, it follows from 49 , that if $\mathbb{E}\|X+Z\|^{2}$ is bounded and if $\mathbb{E}\left\|X+\hat{Z}_{n}\right\|^{2}$ is bounded with high probability, then the decay rate of the error in the entropy follows directly from the decay rate of $W_{2}\left(\mu_{X+Z}, \mu_{X+\hat{Z}_{n}}\right)$. In turn, the dependence of this upper bound on $\mu_{X}$ can be washed out since Wasserstein distances are non-increasing under convolution operations, and so $W_{2}\left(\mu_{X+Z}, \mu_{X+\hat{Z}_{n}}\right) \leq W_{2}\left(\mu_{Z}, \mu_{\hat{Z}_{n}}\right)$ (as any coupling of $\left(Z^{*}, \hat{Z}_{n}^{*}\right)$ defines a coupling $\left(X+Z^{*}, X+\hat{Z}_{n}^{*}\right)$ for $\left.\left(X+Z, X+\hat{Z}_{n}\right)\right)$. Given such a bound, the proof is then completed by using known results $[23]$ on $\mathbb{E}\left[W_{2}\left(\mu_{Z}, \mu_{\hat{Z}_{n}}\right)\right]$ and establishing concentration to this expectation. The actual proof follows these lines, and uses a truncation argument on the norm of the noise $\|Z\|$ to establish such properties under the milder premise of the theorem.

Relation to source coding (quantization): From its definition, one can anticipate that the Wasserstein distance of order $p$ would be useful in bounding $p$ th moment of empirical errors. Indeed, a classic result of Pollard [91] relates the error of a quantizer to the minimal Wasserstein distance between the distribution of the source and any other distribution supported on a finite number of points equal to the cardinality of the codebook (see also [66. Sec. 2.2.1]). To wit, let $\mathcal{C}(m):=\left\{C \subset \mathbb{R}^{d}:|C|=m\right\}$ be all possible codebooks of cardinality $m \in \mathbb{N}$, and let $\mathcal{N}(m):=\left\{\nu \in \mathcal{P}\left(\mathbb{R}^{d}\right):|\operatorname{supp}\{\nu\}|=m\right\}$. Then, for $p \geq 1$ it holds that

$$
\inf _{C \in \mathcal{C}(m)} \mathbb{E} \int \min _{x \in C}\|x-Z\|^{p} \cdot \mathrm{d} \mu_{Z}=\inf _{\nu \in \mathcal{N}(m)} W_{p}^{p}\left(\nu, \mu_{Z}\right) .
$$

In [23], the convergence rates as a function of $m$ of the Wasserstein distance to $\mu_{Z}$ were studied for the empirical measure $\mu_{\hat{Z}_{m}}$ rather than for the optimal density, i.e., $\mathbb{E}\left[W_{p}^{p}\left(\mu_{\hat{Z}_{m}}, \mu_{Z}\right)\right]$ instead of the r.h.s. of 50p. So, therein the error of the density estimator is controlled by a Wasserstein distance. Our proof here, exhibits another use of the empirical Wasserstein distance, as a uniform bound on the error of an entropy estimator. Thus, despite what might have been apparent from its definition, the role of Wasserstein distance goes beyond bounds on the $p$ th norm.

\section{A Finite Support Class of Input Distributions}

The second class of input distributions is similar to (40), and seeks to only optimize weights. That is, a codebook $C=\left\{x_{j}\right\}_{j \in[m]} \subset \mathbb{R}^{d}$ is chosen in advance, and the class of input distributions is

$$
\mathcal{P}_{C}^{* *}:=\left\{\mu_{X}=\sum_{j=1}^{m} a_{j} \delta_{x_{j}}: \boldsymbol{a} \in \mathbb{A}^{m-1}\right\} .
$$

Thus, an input distribution from $\mathcal{P}_{C}^{* *}$ is equivalent to a probability vector $\boldsymbol{a} \in \mathbb{A}^{m-1}$. The problem of maximizing $h(X+Z)$ over $\mu_{X} \in \mathcal{P}_{C}^{* *}$ is a concave optimization problem over the convex set $\mathbb{A}^{m-1}$ which, as said, can also be 
solved efficiently using the Blahut-Arimoto algorithm [3], [9]. As we show next it can also be approximated when the noise distribution is unknown. To this end, consider a kernel $\kappa: \mathbb{R}^{d} \rightarrow \mathbb{R}$, and denote by $\kappa_{\theta, z}$ its shift by $z \in \mathbb{R}^{d}$ followed by scaling of $\theta \in \mathbb{R}_{+}$, to wit, $\kappa_{\theta, z}(x):=\kappa\left(\frac{x-z}{\theta}\right)$. The learning procedure is obtained by maximizing $h\left(X+\tilde{Z}_{n}\right)$ where $\tilde{Z}_{n}=\hat{Z}_{n}+V$ and $V \sim \kappa_{\theta, 0}$. In other words, $\mu_{\tilde{Z}_{n}}$ is a KDE of $\mu_{Z}$ of bandwidth $\theta$, i.e.

$$
\mu_{\tilde{Z}_{n}}(z)=\frac{1}{n \theta^{d}} \sum_{i=1}^{n} \kappa\left(\frac{Z_{i}-z}{\theta}\right) .
$$

To state the result, we denote the second-order differential Rényi entropy by $h_{2}(f):=-\log \int f^{2}$. The following theorem is followed by a discussion on its implications:

Theorem 7. Let $C=\left\{x_{j}\right\}_{j \in[m]} \subset \mathbb{R}^{d}$ be given, and let $X_{\boldsymbol{a}} \sim \sum_{j=1}^{m} a_{j} \delta_{x_{j}}$ for $\boldsymbol{a} \in \mathbb{A}^{m-1}$. Let $\boldsymbol{Z}=\left(Z_{1}, \ldots, Z_{n}\right) \stackrel{\text { i.i.d. }}{\sim}$ $\mu_{Z}$, where $\left|h_{2}\left(\mu_{Z}\right)\right| \leq A_{Z, 2}$. Let $\hat{Z}_{n} \sim \frac{1}{n} \sum_{i=1}^{n} \delta_{Z_{n}}$ and assume that $\mu_{Z}$ is estimated by $\tilde{Z}_{n} \sim \frac{1}{n \theta^{d}} \sum_{i=1}^{n} \kappa_{\theta, Z_{i}}$ where the kernel density satisfies $\left|h_{2}(\kappa)\right| \leq A_{\kappa, 2}$. Then, there exists an absolute constant $c>0$ and $n_{0} \in \mathbb{N}$ which depend on $\left(A_{\kappa, 2}, A_{Z, 2}\right)$ such that for any given $\delta>0$,

$$
\max _{\boldsymbol{a} \in \mathbb{A}^{m-1}}\left|h\left(X_{\boldsymbol{a}}+Z\right)-h\left(X_{\boldsymbol{a}}+\tilde{Z}_{n}\right)\right| \leq \max _{\boldsymbol{a} \in \mathbb{A}^{m-1}} \mathbb{E}\left[\left|h\left(X_{\boldsymbol{a}}+Z\right)-h\left(X_{\boldsymbol{a}}+\tilde{Z}_{n}\right)\right|\right]+\Delta
$$

with probability larger than $1-\delta$, where

$$
\Delta \leq c\left(d \log \frac{1}{h}+\log n+\log m\right) \sqrt{\frac{\log \frac{1}{\delta}+m \log m+m \log n}{n}} .
$$

Convergence rates and choice of bandwidth: As evident, the bound on the convergence rate in the r.h.s. of (53) depends on two terms. The convergence rate of the first term hinges on the ability to properly estimate $h\left(X_{\boldsymbol{a}}+Z\right)$ for any $\boldsymbol{a} \in \mathbb{A}^{m-1}$, and more importantly, for any possible noise distribution. This is typically assured by smoothness assumptions on the possible noise densities, and requires choosing the bandwidth to be $\theta=n^{-r}$ for some $r>0$ that depends on the dimension $d$ and smoothness defining parameters. With this choice, the exponent $r$ only multiplicatively affects the redundancy term $\Delta$ via $d \log \frac{1}{\theta}+\log n=(d r+1) \log n$. For concreteness, we may consider the family of noise densities defined by Lipschitz balls, as proposed and analyzed in [46]. Given a smoothness parameter $s \geq 0$ and $r=\lceil s\rceil$, a norm parameter $p \in[2, \infty)$, and the dimension $d$, a Lipschitz norm is defined as follows:

$$
\begin{gathered}
\|\mu\|_{\text {Lip }}:=\|\mu\|_{L_{p}}+\sup _{t>0} t^{-s} \omega_{r}(\mu, t)_{p} \\
\omega_{r}(\mu, t)_{p}:=\sup _{e \in \mathbb{R}^{d}:\|e\| \leq 1}\left\|\Delta_{t e}^{r} \mu\right\|_{L_{p}} \\
\Delta_{\theta}^{r} \mu(z)=\sum_{k=0}^{r}(-1)^{r-k}\left(\begin{array}{l}
r \\
k
\end{array}\right) \mu\left(z+\left(k-\frac{r}{2}\right) \theta\right), \quad z \in \mathbb{R}^{d}
\end{gathered}
$$

where $\|\mu\|_{L_{p}}=\mathbb{E}^{1 / p}\left[\|X\|^{p}\right]$ with $X \sim \mu$ is the $L_{p}$ norm. The definition $\|\mu\|_{\text {Lip }}$ indeed induces a norm, and so, it is specifically convex. This can be seen from the observation is that $\Delta_{\theta}^{r}$ is a linear operator, and that since $L_{p}$ norm 
are convex functions, it holds for any mixture $\mu=q \mu_{1}+(1-q) \mu_{2}$ that

$$
\omega_{r}(\mu, t)_{p} \leq \sup _{e \in \mathbb{R}^{d}:\|e\| \leq 1} q \cdot\left\|\Delta_{t e}^{r} \mu_{1}\right\|_{L_{p}}+(1-q)\left\|\Delta_{t e}^{r} \mu_{1}\right\|_{L_{p}} \leq q \cdot \omega_{r}\left(\mu_{1}, t\right)_{p}+(1-q) \omega_{r}\left(\mu_{2}, t\right)_{p}
$$

and consequently

$$
\|\mu\|_{\text {Lip }} \leq q\left\|\mu_{1}\right\|_{\text {Lip }}+(1-q)\left\|\mu_{2}\right\|_{\text {Lip }} \leq\left\|\mu_{1}\right\|_{\text {Lip }} \vee\left\|\mu_{2}\right\|_{\text {Lip }}
$$

Generalizing this, we obtain that the density of $X_{\boldsymbol{a}}+Z$ is only smoother than that of $Z$ in the sense that $\left\|\mu_{X_{a}+Z}\right\|_{\text {Lip }} \leq$ $\left\|\mu_{Z}\right\|_{\text {Lip. }}$ Now, following [46], consider the set of densities

$$
\mathcal{B}_{s, p, d}(L):=\left\{\mu:\|\mu\|_{\text {Lip }} \leq L, \operatorname{supp}\left(\mu_{Z}\right) \subseteq[0,1]^{d}\right\}
$$

Given this definition, it was shown in [46, Thm. 3] that whenever the kernel $\kappa$ satisfies several regularity assumptions (non-negativity, unit total mass, zero mean, finite second moment, and compact support ; see [46, Assumption 1]) an upper bound on the entropy estimator can be obtained as follows. Assuming that $s \in(0,2]$, and $p \geq 2$, there exists a constant $C>0$ independent of $n, L$ such that if $L \leq n^{s / d}$ and $\theta \asymp(L n)^{-1 /(s+d)}$ then

$$
\sup _{\mu \in \mathcal{B}_{s, p, d}(L)} \mathbb{E}\left[\left|h(\mu)-h\left(\mu_{\tilde{Z}_{n}}\right)\right|\right] \leq C\left(n^{-s /(s+d)} L^{d /(s+d)}+n^{-1 / 2} \cdot \log L\right)
$$

where $\mu_{\tilde{Z}_{n}}$ is the $\mathrm{KDE}$ as in 52,12 As discussed above, if $\mu_{Z} \in \mathcal{B}_{s, p, d}(L)$ then $\mu_{X+Z} \in \mathcal{B}_{s, p, d}(\tilde{L})$ holds too for some $\tilde{L}$ (with possibly larger support, which can be re-normalized at the expense of a multiplicative factor in $L$ that results $\tilde{L}$ ). Thus, the convergence rates of the first term on the r.h.s. of 53 can be bounded as in 61 . Consequently, for a fixed $L$, the rate in 53 is determined by the first term on the r.h.s., and equals to $O\left(n^{-s /(s+d)}\right)$. This is valid for any $d \geq 1$ and since $s \in(0,2]$ may amount to better rates than $\tilde{O}\left(n^{-1 / d}\right)$ obtained in Theorem 6 whenever $s>\frac{d}{d-1}$ (assuming $d \geq 5$ so that Theorem 6 is valid). Nonetheless, the rate $O\left(n^{-s /(s+d)}\right)$ is fastest when $s=2$ and then $O\left(n^{-2 /(2+d)}\right)$ which, similarly to the $O\left(n^{-1 / d}\right)$ rate of Theorem 6 also requires a number of samples which is exponential in the dimension.

Proof outline: The proof is based on a bound on $\left|h\left(X_{\boldsymbol{a}}+Z\right)-h\left(X_{\boldsymbol{b}}+Z\right)\right|$ for $\boldsymbol{a}, \boldsymbol{b} \in \mathbb{A}^{m-1}$ in terms of the total variation and chi-square divergence between $\boldsymbol{a}$ and $\boldsymbol{b}$ (Lemma 24). This bound is used to show a boundeddifference inequality for $\left|h\left(X_{\boldsymbol{a}}+Z\right)-h\left(X_{\boldsymbol{a}}+\tilde{Z}_{n}\right)\right|$ when one of the samples in $\boldsymbol{Z}$ is changed and consequently establishes the concentration of this quantity to its mean for a given $\boldsymbol{a} \in \mathbb{A}^{m-1}$ via McDiarmid's inequality. Then, uniform concentration over $\mathbb{A}^{m-1}$ is established by a covering argument of the simplex w.r.t. the total variation and chi-square divergence.

\footnotetext{
${ }^{12}$ In fact, in [46. Thm. 3], the expectation in the left-hand side of 61 is replaced by $\mathbb{E}^{1 / 2}\left[\left(h(\mu)-h\left(\tilde{\mu}_{n}\right)\right)^{2}\right]$ and the corresponding statement is stronger. Moreover [46 Thm. 3] shows that the rate on the r.h.s of (61) is sub-optimal, and by using a more sophisticated estimator than 52, one can improve the $n^{-s /(s+d)}$ term in 61 to $(n \log n)^{-s /(s+d)}$, and also that this rate is minimax optimal. However, uniform convergence as in Theorem 7 is more difficult to obtain for that estimator.
} 


\section{EXPERIMENTS}

\section{A. Alternating Optimization for Surrogate Error Probability}

Let us denote the projection operator $\Psi_{r}^{d}: \mathbb{R}^{d} \mapsto \mathcal{B}^{d}(r)$ by $\Psi_{r}^{d}(x)=\frac{(\|x\| \wedge r)}{\|x\|} \cdot x$. For brevity, the subscript $r$ will be omitted when $r=1$. This operator will be used to truncate Gaussian noise to have bounded norm so that Theorem 3 will be valid, but with the expectation that it has little affect on the results. For purpose of illustration we begin with a simple example for $d=2$, and then experiment more extensively with in a more complicated setting 13

1) Two-dimensional Gaussian noise: We assume the noise is (projected) Gaussian $Z \sim \Psi^{d}(N(0, K))$, with covariance matrix $K \in \mathbb{S}_{+}^{d}$ unknown to the learner. It is assumed that $K$ is chosen such that the projection operation only affects $Z$ with low probability and thus rather negligible. We further assume that the codewords of the initial codebook $C^{(0)}=\left(X_{1}^{(0)}, \ldots, X_{m}^{(0)}\right)$ are randomly drawn $X_{j}^{(0)} \sim \Psi_{r_{x}}^{d}\left(N\left(0, \frac{r_{x}^{2}}{\phi_{x} d} \cdot I_{d}\right)\right)$ for $j \in[m]$ mutually independently, where $r_{x}>0$ is the amplitude constraint, and $\phi_{x}$ is similarly chosen such that projection occur with low probability and does not significantly affect the random codebook. With this choice, the mutual information between $X \in \mathbb{R}^{d}$ and $Y=X+Z \in \mathbb{R}^{d}$ is approximated by

$$
I\left(r_{x}\right):=I(X ; Y)=\frac{1}{2} \log \frac{\operatorname{det}\left(\frac{r_{x}^{2}}{\phi_{x} d} \cdot I_{d}+K\right)}{\operatorname{det}(K)} .
$$

The expression in (62) is only approximation since the input distribution is not exactly Gaussian due to the projection operation, but it is only used to roughly gauge the required power for various codebook sizes. For a codebook of size $m$, the minimal required power to obtain negligible error probability in case coding across multiple realizations are allowed is (approximately) $r_{\min }:=\min \{r>0: I(r)>\log m\}$. Since we are considering only a single use of the $d$-dimensional channel, we choose the input power to be $r_{x}=\sqrt{\Gamma} \cdot r_{\min }$ where $\Gamma>0$ is the so-called gap-tocapacity [35, Ch. 4]. The inverse covariance matrix is initialized as the inverse of the empirical covariance matrix over the first $d$ samples, to wit, $S^{(0)}=\cdots=S^{(d)}=\left(\sum_{i=1}^{d} z_{i} z_{i}^{T}\right)^{-1}$. The parameters used in the experiment are detailed in Table I in Appendix B. Fig. 1 displays the output codebook $C^{(n)}$ of a run of Algorithm 1 on a realization of $n$ noise samples, as well as the Voronoi regions determined by this codebook, and the minimal Mahalanobis distance rule w.r.t. the output inverse covariance matrix $S^{(n)}$. In addition, the figure also displays the noise samples used for training superimposed on each of the codewords, i.e., $X_{j}+Z_{i}$ for all $j \in[m]$ and $i \in[n]$ (with slightly different color tone for any $j \in[m])$. Fig. 2 displays the evolution of the empirical error $\overline{\mathbf{p}}_{\boldsymbol{Z}^{(i)}}\left(C^{(i)}, S^{(i)}\right)$ where $\boldsymbol{Z}^{(i)}=\left(Z_{1}, \ldots, Z_{i}\right)$ are the noise samples used up to iteration $i \in[n]$, and the evolution of $\overline{\mathbf{p}}_{\tilde{\boldsymbol{Z}}}\left(C^{(i)}, S^{(i)}\right)$ where $\tilde{\boldsymbol{Z}}=\left(\tilde{Z}_{1}, \ldots, \tilde{Z}_{\tilde{n}}\right)$ are validation samples. The samples $\tilde{\boldsymbol{Z}}$ are drawn independently of the training samples $\boldsymbol{Z}$, and for simplicity only drawn once for all iterations. The validation average error probability serves as a proxy to the statistical average loss $\overline{\mathbf{p}}_{\tilde{Z}}\left(C^{(i)}, S^{(i)}\right) \approx \overline{\mathbf{p}}_{\mu}\left(C^{(i)}, S^{(i)}\right)$. The same evolution is shown for the standard error probability loss function. The results displayed in those figures are for a single "successful", yet representative, run

${ }^{13}$ Matlab code which implements the learning algorithms proposed in the paper and use in the experiments is available at http://drive. google.com/open?id=1YSX0cnac7zpviQxjCx_cdjT_zW6zVx3e and documented at the end of this manuscript. 


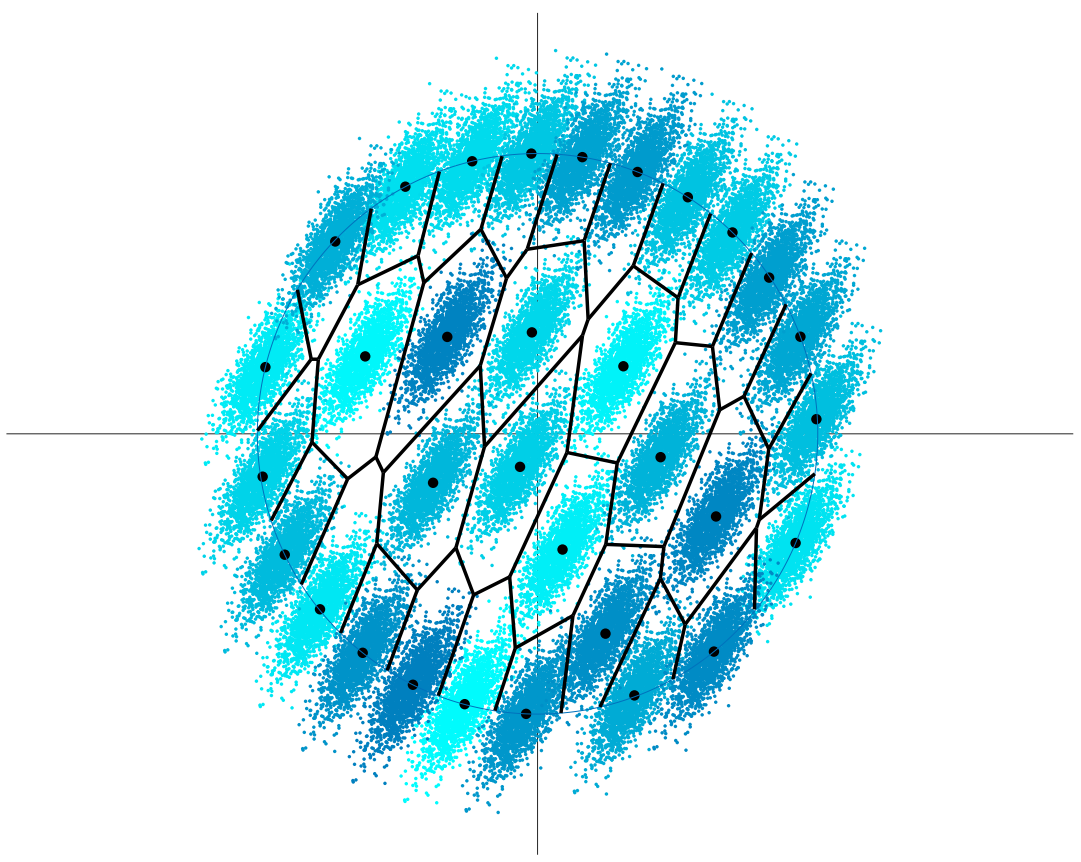

Figure 1. A scatter plot of the final codebook $C^{(n)}$ (black filled circles), Voronoi regions w.r.t. the Mahalanobis distance $\|\cdot\|_{S}(n)$, and noise training samples superimposed on the final codebook.

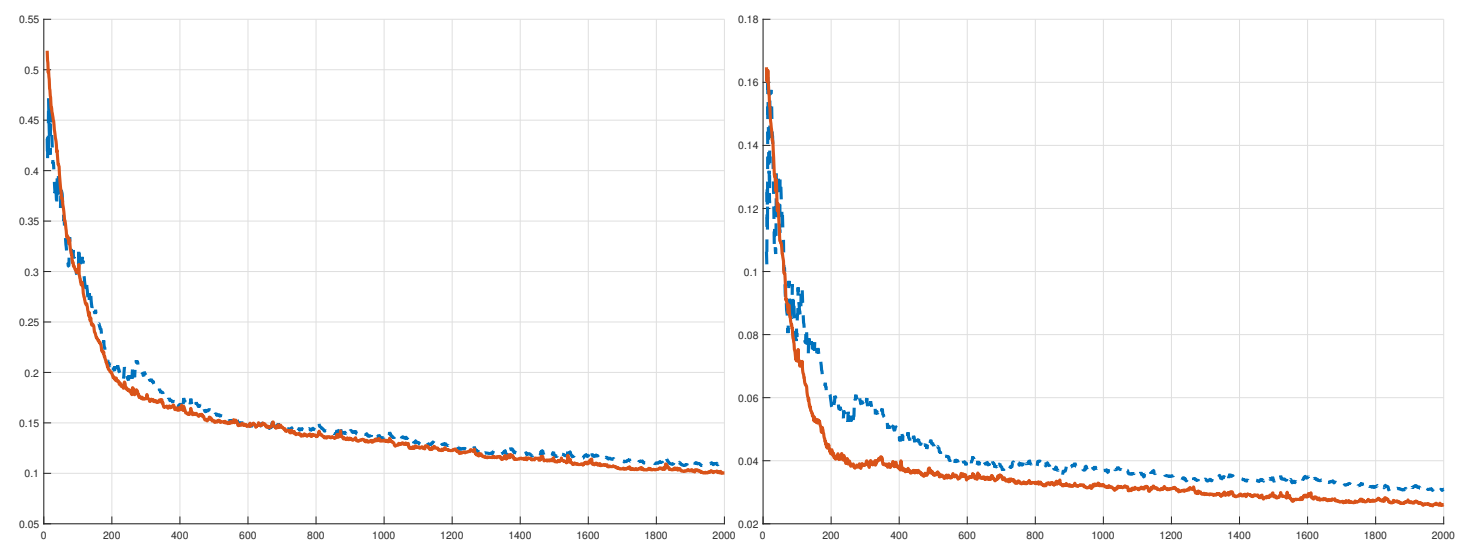

Figure 2. Left: surrogate error probability $\overline{\mathbf{p}}_{Z^{(i)}}\left(C^{(i)}, S^{(i)}\right)-$ blue dashed, $\overline{\mathbf{p}}_{\tilde{\boldsymbol{Z}}}\left(C^{(i)}, S^{(i)}\right)$ - red solid. Right: error probability $\mathbf{p}_{\boldsymbol{Z}^{(i)}}\left(C^{(i)}, S^{(i)}\right)$ - blue dashed, $\mathbf{p}_{\tilde{Z}}\left(C^{(i)}, S^{(i)}\right)$ - red solid

of the algorithm, in which the error probability on the validation samples has decreased by a factor of 4 after about $n=200$ iterations and samples.

2) Gaussian Mixture noise: We consider next the scenario in which the noise $Z$ is comprised of interference from other digital communication transmitters, in addition to standard Gaussian noise. A common strategy is to avoid special processing of this interference and treat such interference as noise. It is then also common to assume that the noise distribution is Gaussian (perhaps with an unknown covariance matrix), which is justified via the central-limit theorem (in case of large number of interferers), as well as the extremal properties of the Gaussian distribution (such as minimizing capacity under covariance constraint, e.g. [57], [90], [24, Lemma II.2]). The learning-based framework proposed here provides a compromise between a worst-case assumption of Gaussian distribution, and a 
detailed parametric characterization of the noise.

To experiment with this approach, we consider $k$ interfering transmitters, where the $l$ th transmitter sends a binary signal $R_{l} \sim$ Uniform $\{-1,1\}$, i.i.d. for $l \in[s]$, such that the noiseless interference from transmitted $l$ is received as $R_{l} \cdot v_{l}$ where $v_{l} \in \mathbb{R}^{d}$. The noise distribution thus assumed to satisfy

$$
Z \stackrel{d}{=} \Psi^{d}\left[\sqrt{\alpha}\left(\sum_{l=1}^{s} R_{l} \cdot v_{l}+W\right)\right]
$$

where $W \sim N\left(0, K_{W}\right) \Perp\left(R_{1}, \ldots, R_{s}\right)$ with $K_{W} \in \mathbb{S}_{+}^{d}$ being the covariance matrix of the pure Gaussian noise. The covariance matrix of the noise before projection is then given by $K=\alpha \sum v_{l} \cdot v_{l}^{T}+\alpha K_{W}$, and $\alpha$ is determined so that the maximal eigenvector of $K$ is at most $\frac{1}{\chi_{z}}$ for some parameter $\chi_{Z}>0$ (so that the projection does not significantly affect the noise distribution).

We again assume initial random (projected) Gaussian codebook, where $I(r)$ gauges the gap-to-capacity as before 14 The experiment parameters are detailed in Table I in Appendix B. Fig. 3 shows graphs that track the evolution of the loss over iterations, in a similar fashion to Fig. 2. However, in Fig. 3 the losses are averaged over multiple noise distributions and multiple runs in the following way. First, a noise distribution is chosen where $\left\{v_{l}\right\}$ are random and chosen as $V_{l} \sim N\left(0, I_{d}\right)$, and $K_{W}$ is randomly chosen from the Wishart distribution, i.e., $K_{W}=Q Q^{T}$ where the the $d^{2}$ entries of $Q \in \mathbb{S}^{d}$ are independent $Q_{i_{1} i_{2}} \sim N(0,1)$. Then, for each realization of noise distribution, multiple runs were performed, where in each run a noise realization (both for training and validation) and a random codebook is drawn, independent of all other runs. Fig. 3 displays an averaging and a 0.8-quantile of $10^{3}$ runs where the noise distribution was re-drawn every 10 runs. It is apparent that both type of loss functions follow the same trend and the reduction in the surrogate error probability leads to a reduction in the ordinary error probability. Convergence is achieved by a few hundred samples. It can also be observed that the 0.8-quantile is typically less than the average. This indicates that the events where no convergence is achieved lead to high error, and so can easily be detected, after $100-200$ samples, which marginally increases the convergence time. We also remark that the step-sizes are chosen to be the same for all iterations, and were not thoroughly optimized. In general, the convergence time is longer as the constellation size is bigger. However, the required number of samples (or iterations) required for convergence does not seem to be significantly vary from the surrogate and standard error probability loss functions. To further inspect the dependency on the codebook size, we denote the generalization error for the surrogate and the standard loss functions, respectively, as explicit functions of the codebook size $m$ :

$$
\bar{g}_{m}^{(i)}:=\left|\overline{\mathbf{p}}_{\tilde{\boldsymbol{Z}}}\left(C^{(i)}, S^{(i)} \mid m\right)-\overline{\mathbf{p}}_{\boldsymbol{Z}}\left(C^{(i)}, S^{(i)} \mid m\right)\right|
$$

and

$$
g_{m}^{(i)}:=\left|\mathbf{p}_{\tilde{\boldsymbol{Z}}}\left(C^{(i)}, S^{(i)} \mid m\right)-\mathbf{p}_{\boldsymbol{Z}}\left(C^{(i)}, S^{(i)} \mid m\right)\right| .
$$

\footnotetext{
${ }^{14}$ We use the same metric even though the noise is not Gaussian because Gaussian noise leads to the minimal capacity under a noise covariance constraint.
} 


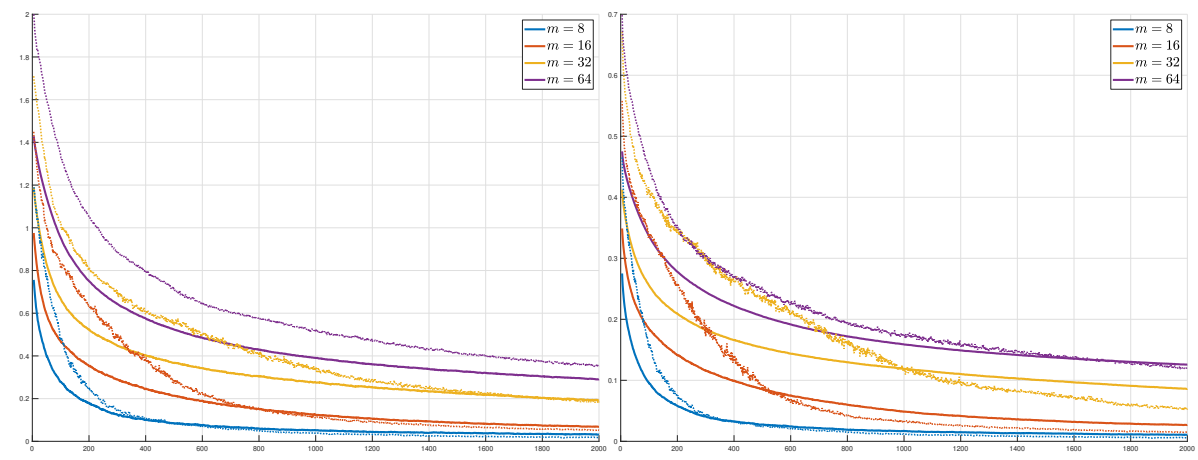

Figure 3. Surrogate $\overline{\mathbf{p}}_{\tilde{\boldsymbol{Z}}}\left(C^{(i)}, S^{(i)}\right)$ (left) and standard $\mathbf{p}_{\tilde{\boldsymbol{Z}}}\left(C^{(i)}, S^{(i)}\right)$ (right) error probability. Averaged over $10^{3}$ experiments - solid, 0.8-quantile - dotted.

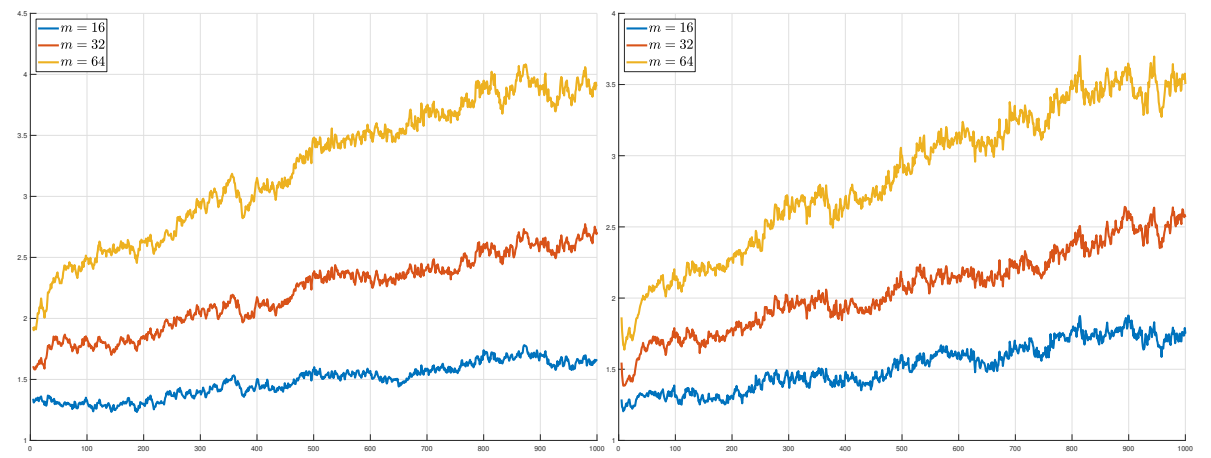

Figure 4. Surrogate $\bar{g}_{m}^{(i)} / \bar{g}_{8}^{(i)}$ (left) and standard $g_{m}^{(i)} / g_{8}^{(i)}$ (right) generalization error ratio, averaged over $10^{3}$ experiments.

The dependency on the codebook size can then be found by the ratios $g_{m}^{(i)} / g_{m_{0}}^{(i)}$ and $\bar{g}_{m}^{(i)} / \bar{g}_{m_{0}}^{(i)}$ for various values of $m$. In Fig. 4, these ratios are computed with $m_{0}=8$, and roughly shows that the dependency of the generalization error on the codebook size is not different between the surrogate and ordinary error probability. In fact, it seems that that the dependency is close to square-root in $m$, as was theoretically obtained for the surrogate error probability (Theorem 3), rather than to the linear dependence theoretically obtained for the standard error probability (Theorem 11. This can be attributed to the fact that Theorem 1 states a generalization bound which assumes an arbitrary noise distribution, and the worst case distribution can be significantly worse than the average-case distribution, or worse than the worst-case distribution within a family of structured distributions such as the Gaussian mixture.

\section{B. Gibbs Algorithm}

We repeat the Gaussian mixture noise experiment from Section VI-A2, Here there is no need to constraint the noise to $\mathcal{B}^{d}(1)$ and the codebook to $\mathcal{B}^{d}\left(r_{x}\right)$ and so we omit the projection operation. We choose the reference measure $Q$ to be the standard Lebesgue measure, so that the codebook at each stage is only chosen based on its error probability and not affected by other factors such as its average power. The decoder is the standard minimum Euclidean decoder. To facilitate the computational load of the Gibbs algorithm we take a memoizationbased approach which is detailed in Appendix C. As evident from Fig. 5, practically there is no reason to increase $\beta$ beyond $\beta=1000$, and the loss of reducing $\beta$ beyond that by a factor of 10 is rather mild. The question 

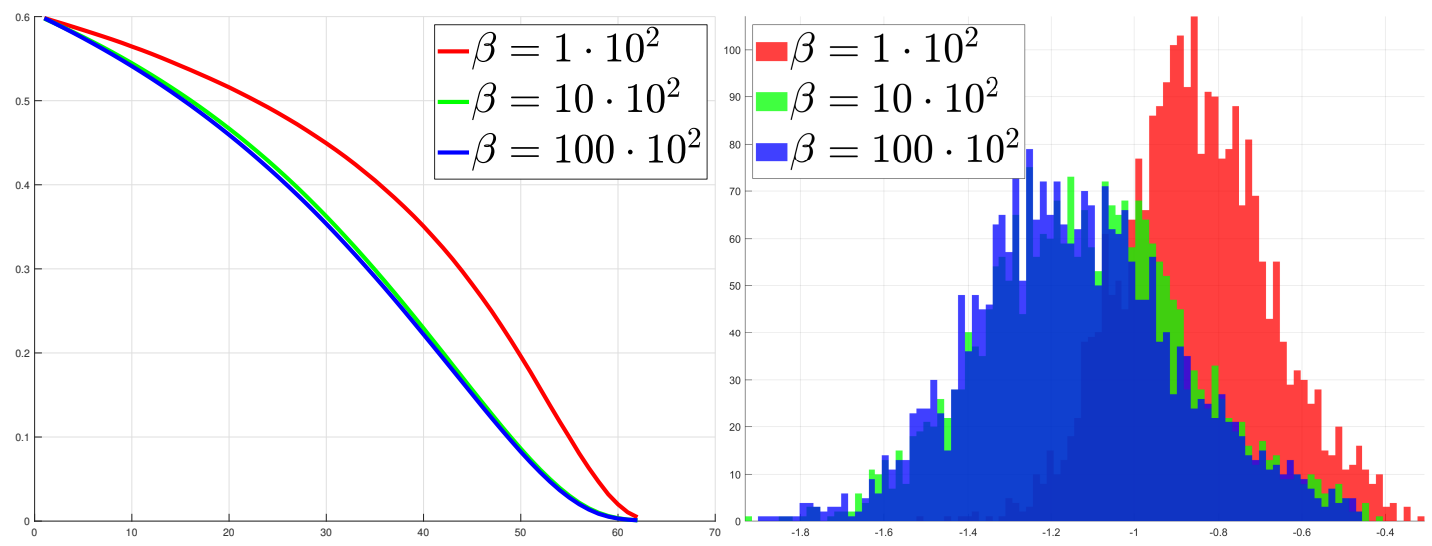

Figure 5. Left: error probability on the validation data $\mathbf{p}_{\tilde{Z}}\left(C^{(i)}\right)$ per iteration - averaged over $2.5 \cdot 10^{3}$ trials. Right: $\log$ error probability on the validation data $\log \mathbf{p}_{\tilde{Z}}\left(C^{(i)}\right)$ - histogram over $2.5 \cdot 10^{3}$ trials for codebooks of size $m=32$.

whether using finite $\beta$ is merely a theoretical tool to prove generalization bounds or useful in practice remains open. In principle, the theoretical bounds do not depend on any property of the noise distribution, but rather on the algorithmic stability of the Gibbs algorithm. Thus, the results are applicable to arbitrary noise distribution, no matter how complex. In various other experiments we have conducted with Gaussian mixture, there were cases in which reducing the value of $\beta$ has improved performance, yet not in a very consistent or statistically significant way. A noise distribution which is more intricate than Gaussian mixture might leads to finite values of $\beta$ obtaining better performance, and a finite value of $\beta$ universally "protects" against any possible noise distribution.

\section{SUMmARY AND Future RESEARCH}

We have considered the problem of empirical design of an encoder and a decoder of a communication system operating over an additive noise channel, given samples of the noise. We summarize here the main findings and various open problems for future research.

A bound on the generalization error for the regular error probability loss function was stated, which shows a $O\left(\frac{1}{\sqrt{n}}\right)$ dependency on the number of samples. No structure is assumed on the codebook and in turn the generalization bound depends linearly on the codebook size $m$. A goal for future research is to settle the dependency of the generalization bound on the allowed codebook structure and size, via a possibly refined generalization bound and a proper converse result. Another possibility of obtaining convergence rates faster than $O\left(\frac{1}{\sqrt{n}}\right)$ is to make assumptions on the noise distribution. Such rates were established for empirical design of quantizers in [2], [67], [68]. Future research could derive analogous conditions and bounds for the problem studied in this paper.

It was further shown that the use of a surrogate loss function to the standard error probability loss function improves the dependency of the generalization error bound on the number of codewords from linear to (nearly) square root. As evident from the proof, this property essentially follows from the continuity of the loss function in the design parameters - the codebook and the inverse covariance matrix. Furthermore, an alternating optimization algorithm was proposed to minimize the empirical loss. At each iteration, a "local" approximation of the loss 
function ${ }^{15}$ is obtained by "freezing" the nearest neighbors for each codeword in the current codebook and decoder, and the iteration is completed by optimizing this upper bound. We expect that similar techniques involving surrogate loss functions and their local approximation could be useful (perhaps with some innovation) to obtain generalization bounds and empirical loss minimization algorithms for much more complicated scenarios, for example, decoders which are based on DNN. A goal for future research is to develop and analyze such algorithms. Furthermore, we have not provided a dedicated analysis of the alternating-minimization SGD algorithm, but rather rely on the general uniform convergence bound for the surrogate loss function. The uniform convergence bound is oblivious to the way the algorithm explore the possible input distributions (based on the noise samples), and thus could be pessimistic when applied to the SGD algorithm. In this case, various stability properties (e.g., [13], [95]) could be used to obtain sharper generalization bounds, as was done, e.g., in [47].

Another possibility for future research is the analysis of different loss functions. For example, the cross-entropy loss function is a commonly used surrogate in the practical learning-based design of end-to-end communication systems (e.g., [31], [87], [121]). Similarly, to the surrogate loss function studied here, it also directly bounds the standard error probability loss function via Pinsker's inequality. However, its analysis would differ from the analysis in this paper mainly as it requires considering a different class of decoders - those which output a posterior probability distribution on the messages given the channel output. In addition, the cross entropy is not a bounded loss function, and so analyzing it it requires more elaborate methods compared to the ones used in this paper.

We have then considered a Gibbs algorithm that expurgates large codebooks in order to obtain smaller codebooks with improved error probability. The randomness in this algorithm, as manifested by the inverse temperature parameter $\beta$, was mainly introduced in order to provably bound the generalization error of such algorithms. In practice, however, most of our experiments have shown that backing $\beta$ from infinity does not improve performance. An open problem is an analytical characterization of the optimal value of $\beta$, and identifying cases in which $\beta<\infty$ improves performance in relevant practical scenarios. Furthermore, finding efficient combinatorial algorithms with theoretical guarantees for the problem of finding an optimal codebook withing a super-codebook is an interesting avenue for future research.

Finally, we have considered the problem of maximizing the mutual information $I(X ; X+Z)$ over the distribution of $X$. We have seen that uniform convergence empirical error rates of $\tilde{O}\left(n^{-1 /(d \vee 4)}\right)$ are possible, under rather general regularity conditions on the distribution of $Z$, and the set of possible distributions for $X$. Some improvement in the rate is possible when the input distribution is constrained to a given support of finite cardinality, and smoothness assumptions on the distribution of $Z$. However, the required number of samples is still exponential in the dimension $d$. Obtaining fast uniform convergence rates seems possible only if the family of input distributions is restricted or the noise distribution is supported on a low-dimensional manifold. This is left for further research. From a different angle, improved rates can be obtained by considering a specific algorithm. As was discussed, finding the optimal input distribution could be a difficult problem even for a known noise distribution. Thus, typically a specific

\footnotetext{
${ }^{15}$ Which is also a provably upper bound to the loss function.
} 
algorithm is used to approximate the optimal input distribution. It is possible that stability-based analysis would be useful to this setting too.

\section{ACKNOWLEDGMENT}

The support of N. Merhav and the comments of the anonymous reviewers are acknowledged with gratitude.

\section{APPENDIX A}

PROOFS

\section{A. A Preliminary - Uniform Convergence by Rademacher Complexity}

We denote the empirical Rademacher complexity of a set $\mathcal{L}_{n} \subset \mathbb{R}^{n}$ by

$$
\operatorname{Rad}\left(\mathcal{L}_{n}\right):=\frac{1}{n} \mathbb{E}\left[\sup _{l^{n} \in \mathcal{L}_{n}} \sum_{i=1}^{n} R_{i} l_{i}\right],
$$

where $l^{n}:=\left(l_{1}, \ldots l_{n}\right) \in \mathbb{R}^{n}$ and $R^{n}:=\left(R_{1}, \ldots R_{n}\right) \in\{ \pm 1\}^{n}$ are Rademacher random variables (i.e., $R_{i} \sim$ Uniform $\{-1,1\}$, i.i.d.).

Proposition 8. Let $\mathcal{Z}$ be a data samples space and let $\mathcal{H}$ be a hypothesis class. If the loss function $\ell:(\mathcal{H}, \mathcal{Z}) \mapsto \mathbb{R}$ is absolutely bounded $|\ell(h, z)| \leq r$ then

$$
\mathbb{P}_{\boldsymbol{Z}^{\text {i.i.d. }} \sim}\left[\bigcap_{h \in \mathcal{H}}\left\{\left|\mathbb{E}_{Z \sim \mu}[\ell(h, Z)]-\frac{1}{n} \sum_{i=1}^{n} \ell\left(h, Z_{i}\right)\right| \leq A_{n}(\delta)\right\}\right] \geq 1-\delta,
$$

where

$$
A_{n}(\delta):=4 \cdot \mathbb{E}\left[\operatorname{Rad}\left(\mathcal{L}_{\boldsymbol{Z}}\right)\right]+r \sqrt{\frac{2 \ln (2 / \delta)}{n}}
$$

and

$$
\mathcal{L}_{\boldsymbol{Z}}=\left\{\left(\ell\left(h, Z_{1}\right), \ldots, \ell\left(h, Z_{n}\right)\right): h \in \mathcal{H}\right\}
$$

Proof: It is well-established that Rademacher complexity uniformly bounds convergence the deviation of empirical averages from the statistical average [7], and the statement in (67) was made in [105, Thm. 26.5], albeit without the absolute value inside the probability term, and where the first additive term in $A_{n}(\delta)$ is $2 \mathbb{E}\left[\operatorname{Rad}\left(\mathcal{L}_{\boldsymbol{Z}}\right)\right]$. By repeating the same arguments in [105, Ch. 26] that lead to that theorem, while replacing $\mathbb{E}_{Z \sim \mu}[\ell(h, Z)]-$ $\frac{1}{n} \sum_{i=1}^{n} \ell\left(h, Z_{i}\right)$ therein with its absolute value, one can obtain that the probability bound in 67 is valid when is $A_{n}(\delta)$ replaced with

$$
\tilde{A}_{n}(\delta):=\frac{2}{n} \cdot \mathbb{E}\left[\sup _{l^{n} \in \mathcal{L}_{Z}}\left|\sum_{i=1}^{n} R_{i} l_{i}\right|\right]+r \cdot \sqrt{\frac{2 \ln (2 / \delta)}{n}} .
$$


But, using the notation $-\mathcal{L}_{Z}:=\left\{\left(-\ell\left(h, Z_{1}\right), \ldots,-\ell\left(h, Z_{n}\right)\right): h \in \mathcal{H}\right\}$ we have that $\tilde{A}_{n}(\delta) \leq A_{n}(\delta)$ since for any given $\boldsymbol{Z} \in\left(\mathbb{R}^{d}\right)^{n}$

$$
\begin{aligned}
& \mathbb{E}\left[\sup _{l^{n} \in \mathcal{L}_{Z}}\left|\sum_{i=1}^{n} R_{i} l_{i}\right|\right]=\mathbb{E}\left[\left\{\sup _{l^{n} \in \mathcal{L}_{Z}: \sum_{i=1}^{n} R_{i} l_{i}>0} \sum_{i=1}^{n} R_{i} l_{i}\right\} \vee\left\{\sup _{l^{n} \in \mathcal{L}_{Z}: \sum_{i=1}^{n} R_{i} l_{i}<0}-\sum_{i=1}^{n} R_{i} l_{i}\right\}\right] \\
& \leq \mathbb{E}\left[\sup _{l^{n} \in \mathcal{L}_{Z}: \sum_{i=1}^{n} R_{i} l_{i}>0} \sum_{i=1}^{n} R_{i} l_{i}+\sup _{l^{n} \in-\mathcal{L}_{Z}: \sum_{i=1}^{n} R_{i} l_{i}>0} \sum_{i=1}^{n} R_{i} l_{i}\right] \\
& \leq \mathbb{E}\left[\sup _{l^{n} \in \mathcal{L}_{Z}} \sum_{i=1}^{n} R_{i} l_{i}+\sup _{l^{n} \in-\mathcal{L}_{Z}} \sum_{i=1}^{n} R_{i} l_{i}\right] \\
& =\mathbb{E}\left[\sup _{l^{n} \in \mathcal{L}_{Z}} \sum_{i=1}^{n} R_{i} l_{i}\right]+\mathbb{E}\left[\sup _{l^{n} \in \mathcal{L}_{Z}} \sum_{i=1}^{n}\left(-R_{i}\right) l_{i}\right] \\
& =2 \operatorname{Rad}\left(\mathcal{L}_{\boldsymbol{Z}}\right) \text {, }
\end{aligned}
$$

where in the last equality we have used $R_{i} \stackrel{d}{=}-R_{i}$.

\section{B. The Proofs of Theorem 1 and Proposition 2}

Proof of Theorem 17. For a given training set $\left\{z_{i}\right\}_{i=1}^{n}$, we define the loss vector as

$$
l^{n}(C, S):=\left(\ell\left(C, S, z_{1}\right), \ldots, \ell\left(C, S, z_{n}\right)\right)
$$

and the loss class as $\mathcal{L}_{n}(m):=\left\{l^{n}(C, S): C \in\left(\mathbb{R}^{d}\right)^{m}, S \in \mathbb{S}_{+}^{d}\right\}$. We next bound the Rademacher complexity 66 of $\mathcal{L}_{n}(m)$ and then use Prop. 8. As $\left|\mathcal{L}_{n}(m)\right|<\infty$, Massart's lemma [105, Thm. 26.8] implies that

$$
\operatorname{Rad}\left(\mathcal{L}_{n}(m)\right) \leq \max _{l^{n} \in \mathcal{L}_{n}(m)}\left\|l^{n}-\overline{l^{n}}\right\| \frac{\sqrt{2 \log \left|\mathcal{L}_{n}(m)\right|}}{n} \leq \sqrt{\frac{2 \log \left|\mathcal{L}_{n}(m)\right|}{n}},
$$

where $\overline{l^{n}}=\frac{1}{\left|\mathcal{L}_{n}(m)\right|} \sum_{l^{n} \in \mathcal{L}_{n}(m)} l^{n}$, and the last inequality holds since as $l^{n} \in[0,1]^{n}$. We further bound $\left|\mathcal{L}_{n}(m)\right|$ as follows. A loss vector $l^{n}(C, S)$ is unequivocally determined by $m$ loss vectors

$$
l_{j}^{n}(C, S):=\left(\ell_{j}\left(C, S, z_{1}\right), \ldots, \ell_{j}\left(C, S, z_{n}\right)\right) \in\{0,1\}^{n}
$$

for $j \in[m]$. In turn, each loss vector $l_{j}^{n}(C, S)$ is unequivocally determined by the $m-1$ binary classification vectors

$$
b_{j^{\prime} \mid j}^{n}(C, S)=\left(b_{j^{\prime} \mid j}\left(C, S, z_{1}\right), \ldots, b_{j^{\prime} \mid j}\left(C, S, z_{n}\right)\right)
$$

for $j^{\prime} \neq j$, with

$$
b_{j^{\prime} \mid j}(C, S, z)=\mathbb{1}\left\{\left\|x_{j}-x_{j^{\prime}}\right\|_{S}^{2}+2\left(x_{j}-x_{j^{\prime}}\right)^{T} S z<0\right\} .
$$

Hence, $b_{j^{\prime} \mid j}^{n}(C, S)$ is the result of the binary classifier induced by $\left\{x_{j}, x_{j^{\prime}}\right\}$ and the nearest neighbor decoding rule. Since this classifier is in fact an affine hyperplane, its VC dimension is upper bounded by $d+1$ [105, Thm. 9.3]. 
By the Sauer-Shelah lemma [105, Lemma 6.10], for $n \geq d+1$

$$
\left|\left\{b_{j^{\prime} \mid j}^{n}(C, S): C \in\left(\mathbb{R}^{d}\right)^{m}, S \in \mathbb{S}_{+}^{d}\right\}\right| \leq\left(\frac{e n}{d+1}\right)^{d+1} .
$$

Accounting for all pairs $j, j^{\prime} \in[m]$ we thus obtain

$$
\left|\mathcal{L}_{n}(m)\right| \leq\left(\frac{e n}{d+1}\right)^{m^{2}(d+1)} .
$$

Inserting the bound (82) to (77) and using Prop. 8 completes the proof.

Remark 9. Despite (82) being a crude estimate, other bounding techniques such as using Natarajan dimension for multiclass classification do not yield improved bounds (at least not using using direct arguments). Furthermore, a seemingly natural way of defining a class of classifiers which determine $\ell_{j}(C, S, z)$ is as an intersection of $m$ hyperplanes. For this class, the classic paper [10] (see also [18]) gives a bound of $O((d+1) m \log m)$ which leads to a worse bound than the one obtained here.

We now turn to the proof of Proposition 2 which requires a few lemmas. Let $Q(t):=\mathbb{P}[W>t]$ where $W \sim N(0,1)$ be the Gaussian tail distribution function. The next lemma states the error probability of a codebook of two antipodal codewords.

Lemma 10. Let $d=2$ and consider a codebook of $m=2$ codewords $C=\left(x_{1}, x_{2}\right)$ given by $x_{1}=(\cos \alpha, \sin \alpha)^{T}=$ $-x_{2}$ for some $\alpha \in[0, \pi]$. Parameterize the decoder inverse covariance matrix by

$$
S=\left[\begin{array}{cc}
\cos (\beta) & \sin (\beta) \\
-\sin (\beta) & \cos (\beta)
\end{array}\right]\left[\begin{array}{cc}
s(1) & 0 \\
0 & s(2)
\end{array}\right]\left[\begin{array}{cc}
\cos (\beta) & -\sin (\beta) \\
\sin (\beta) & \cos (\beta)
\end{array}\right]
$$

with $s(1), s(2) \in \mathbb{R}_{+}$and $\beta \in[0, \pi]$. If $Z \sim N\left(\mathbf{0}, \operatorname{diag}\left(\sigma^{2}(1), \sigma^{2}(2)\right)\right)$ then,

$$
\mathbf{p}_{\mu}(C, S)=Q\left(\frac{\cos ^{2}(\alpha+\beta) s(1)+\sin ^{2}(\alpha+\beta) s(2)}{\tau}\right)
$$

where

$$
\begin{aligned}
\tau^{2}\left(\sigma^{2}(1), \sigma^{2}(2), s(1), s(2), \alpha, \beta\right): & =\sigma^{2}(1) \cdot[s(1) \cos (\beta) \cos (\alpha+\beta)+s(2) \sin (\beta) \sin (\alpha+\beta)]^{2} \\
& +\sigma^{2}(2) \cdot[-s(1) \sin (\beta) \cos (\alpha+\beta)+s(2) \cos (\beta) \sin (\alpha+\beta)]^{2} .
\end{aligned}
$$

If $\sigma(1)=\sigma(2) \equiv \sigma$ then $\min _{\alpha, \beta, s(1), s(2)} \mathbf{p}_{\mu}(C, S)=Q\left(\frac{1}{\sigma}\right)$ is achieved with $s(1)=s(2)=1$.

The proof of Lemma 10 is a trivial exercise and thus omitted.

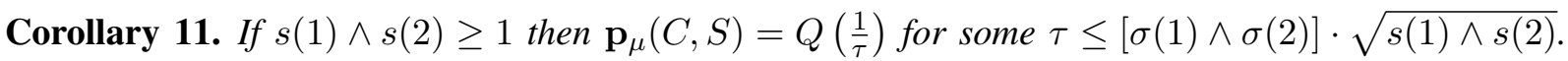

Proof: We consider three cases. First, if $\cos (\alpha+\beta)=0$ and $\sin (\alpha+\beta)=1$, then by (84) and 85), the error 
probability is

$$
\begin{aligned}
\mathbf{p}_{\mu}(C, S) & =Q\left(\frac{1}{\sqrt{\sigma^{2}(1) \sin ^{2}(\beta)+\sigma^{2}(2) \cos ^{2}(\beta)}}\right) \\
& \leq Q\left(\frac{1}{\sigma(1) \vee \sigma(2)}\right) \\
& \leq Q\left(\frac{1}{[\sigma(1) \vee \sigma(2)] \cdot \sqrt{s(1) \vee s(2)}}\right)
\end{aligned}
$$

where the last equality follows since $s(1) \wedge s(2) \geq 1$ is assumed, and since $Q(t)$ is monotonic decreasing in $t$. Second, if $\cos (\alpha+\beta)=1$ and $\sin (\alpha+\beta)=0$ then similar analysis leads to the same result. Third, if both $\cos (\alpha+\beta) \neq 0$ and $\sin (\alpha+\beta) \neq 0$ then since the bound $(84)$ is homogeneous w.r.t. $(s(1), s(2))$, we will obtain the same bound for any $c \cdot(s(1), s(2))$, and thus we may assume that

$$
\cos ^{2}(\alpha+\beta) s(1)+\sin ^{2}(\alpha+\beta) s(2) \geq 1 .
$$

In addition, from 85

$$
\begin{aligned}
\tau^{2} \leq & {\left[\sigma^{2}(1) \vee \sigma^{2}(2)\right] \times } \\
& {\left[s^{2}(1) \cos ^{2}(\beta) \cos ^{2}(\alpha+\beta)+s^{2}(2) \sin ^{2}(\beta) \sin ^{2}(\alpha+\beta)\right.} \\
& \left.+s^{2}(1) \sin ^{2}(\beta) \cos ^{2}(\alpha+\beta)+s^{2}(2) \cos ^{2}(\beta) \sin ^{2}(\alpha+\beta)\right] \\
= & {\left[\sigma^{2}(1) \vee \sigma^{2}(2)\right] \cdot\left[s^{2}(1) \cos ^{2}(\alpha+\beta)+s^{2}(2) \sin ^{2}(\alpha+\beta)\right] } \\
\leq & {\left[\sigma^{2}(1) \vee \sigma^{2}(2)\right][s(1) \vee s(2)] \cdot\left[s(1) \cos ^{2}(\alpha+\beta)+s(2) \sin ^{2}(\alpha+\beta)\right] . }
\end{aligned}
$$

Substituting this bound in (84), and utilizing the monotonicity of the $Q$ function and the assumption (89) results

$$
\begin{aligned}
\mathbf{p}_{\mu}(C, S) & \leq Q\left(\frac{\cos ^{2}(\alpha+\beta) s(1)+\sin ^{2}(\alpha+\beta) s(2)}{\sqrt{\sigma^{2}(1) \vee \sigma^{2}(2)} \cdot \sqrt{s(1) \vee s(2)} \cdot \sqrt{s(1) \cos ^{2}(\alpha+\beta)+s(2) \sin ^{2}(\alpha+\beta)}}\right) \\
& \leq Q\left(\frac{1}{\sqrt{\sigma^{2}(1) \vee \sigma^{2}(2)} \cdot \sqrt{s(1) \vee s(2)}}\right) .
\end{aligned}
$$

The next lemma states two properties related to the $Q(\cdot)$ function.

Lemma 12. $Q(1 / \sqrt{t})$ is convex on $[0,1 / 3]$, and $Q(1 / \sqrt{t})-Q(1 / \sqrt{s}) \geq 0.145 \cdot(t-s)$ for $1 / 6<s<t<1 / 3$.

Proof: For the first property, $\frac{\mathrm{d}}{\mathrm{d} t} Q\left(\frac{1}{\sqrt{t}}\right)=\frac{1}{\sqrt{8 \pi}} e^{-1 /(2 t)} t^{-3 / 2}$ by the Leibniz integral rule, and so

$$
\frac{\mathrm{d}^{2}}{\mathrm{~d} t^{2}} Q\left(\frac{1}{\sqrt{t}}\right)=\frac{1}{\sqrt{8 \pi}} e^{-1 /(2 t)}\left[\frac{1}{2 t^{7 / 2}}-\frac{3}{2 t^{5 / 2}}\right]
$$


which is nonnegative for $t \in[0,1 / 3]$. For the second property, using the first property

$$
\begin{aligned}
Q(1 / \sqrt{t})-Q(1 / \sqrt{s}) & =\int_{s}^{t} \frac{\mathrm{d}}{\mathrm{d} x} Q\left(\frac{1}{\sqrt{x}}\right) \cdot \mathrm{d} x \\
& \geq\left.(t-s) \cdot \min _{\tilde{x} \in[1 / 6,1 / 3]} \frac{\mathrm{d}}{\mathrm{d} x} Q\left(\frac{1}{\sqrt{x}}\right)\right|_{x=\tilde{x}} \\
& =\left.(t-s) \cdot \frac{\mathrm{d}}{\mathrm{d} x} Q\left(\frac{1}{\sqrt{x}}\right)\right|_{x=1 / 6}
\end{aligned}
$$

for which the bound numerically holds.

The following lemma essentially states a large-deviations property of central chi-square random variables.

Lemma 13. Let $\left\{W_{i}, \tilde{W}_{i}\right\}_{i \in[n]}$ be i.i.d., such that $W_{i} \sim N\left(0, \sigma^{2}\right), \tilde{W}_{i} \sim N\left(0, \tilde{\sigma}^{2}\right)$ with $\frac{\sigma^{2}}{\tilde{\sigma}^{2}}=1-t$ for some $t \in(0,1)$ and $W_{i} \Perp \tilde{W}_{i}$ for all $i \in[n]$. Then

$$
\mathbb{P}\left[\sum_{i=1}^{n} W_{i}^{2}>\sum_{i=1}^{n} \tilde{W}_{i}^{2}\right]>\frac{1}{4} \exp \left[-\frac{3}{4} n t^{2}\right] .
$$

Proof: The proof is based on the standard change-of-measure argument. Denote by $\varphi_{\sigma}$ the Gaussian probability measure on the Borel sets of $\mathbb{R}$ of mean zero and variance $\sigma^{2}$, and let $\nu:=\varphi_{\sigma}^{\otimes 2 n}$ and $\tilde{\nu}:=\varphi_{\sigma}^{\otimes n} \otimes \varphi_{\tilde{\sigma}}^{\otimes n}$ (which are probability measures on the Borel sets of $\mathbb{R}^{2 n}$ ). By the tensorization property of the KL divergence and a standard calculation

$$
\mathrm{d}_{\mathrm{KL}}(\nu \| \tilde{\nu})=n \mathrm{~d}_{\mathrm{KL}}\left(\varphi_{\sigma} \| \varphi_{\sigma}\right)+n \mathrm{~d}_{\mathrm{KL}}\left(\varphi_{\sigma} \| \varphi_{\tilde{\sigma}}\right)=\frac{n}{2}\left(\frac{\sigma^{2}}{\tilde{\sigma}^{2}}-1-\log \left(\frac{\sigma^{2}}{\tilde{\sigma}^{2}}\right)\right) .
$$

Let $\left(W_{1} \ldots, W_{n}, \tilde{W}_{1} \ldots, \tilde{W}_{n}\right) \in \mathbb{R}^{2 n}$ be the identity random variable on the space defined above, and define $I=\mathbb{1}\left[\sum_{i=1}^{n} W_{i}^{2}>\sum_{i=1}^{n} \tilde{W}_{i}^{2}\right] \in\{0,1\}$. Under $\nu, I$ is Bernoulli with probability of success $\mathbb{E}_{\nu}[I]=\frac{1}{2}$ (by symmetry), and under $\tilde{\nu}$ it is Bernoulli with probability of success given by $p:=\mathbb{E}_{\tilde{\nu}}[I]$, which is the probability required to be lower bounded. By the data processing inequality for the KL divergence [22, Lemma 4.1], [42, Corollary 5.2.2]

$$
\mathrm{d}_{\mathrm{KL}}(\nu \| \tilde{\nu}) \geq \mathrm{d}_{\mathrm{KL}}\left(\left(\frac{1}{2}, \frac{1}{2}\right) \|(p, 1-p)\right) \geq \frac{1}{2} \log \frac{1}{p}-\log 2
$$

and so $p \geq \frac{1}{4} \exp \left[-2 \mathrm{~d}_{\mathrm{KL}}(\nu \| \tilde{\nu})\right]$ which, along with $[100$, , implies that

$$
\mathbb{P}\left[\sum_{i=1}^{n} W_{i}^{2}(1)>\sum_{i=1}^{n} \tilde{W}_{i}^{2}\right]>\frac{1}{4} \exp \left[-n\left(\frac{\sigma^{2}}{\tilde{\sigma}^{2}}-1-\log \left(\frac{\sigma^{2}}{\tilde{\sigma}^{2}}\right)\right)\right] .
$$

The proof is completed by bounding the exponent in the last bound. Using a second order Taylor approximation of $\log x$ around $x=1$, with a Lagrange form for the remainder, which is valid for, say, $\left[\frac{4}{7}, 1\right]$, we have

$$
\left|\log (x)-\left[(x-1)-\frac{1}{2}(x-1)^{2}\right]\right| \leq \max _{\tilde{x} \in\left[\frac{4}{7}, 1\right]}\left|\left(\frac{1}{3 \tilde{x}^{3}}\right)(x-1)^{3}\right| \leq \frac{1}{4}(x-1)^{2}
$$


and so $\log (x) \geq(x-1)-\frac{3}{4}(x-1)^{2}$ holds for $x \in\left[\frac{4}{7}, 1\right]$. Hence, if $\frac{\sigma^{2}}{\tilde{\sigma}^{2}}=1-t$ then

$$
\frac{\sigma^{2}}{\tilde{\sigma}^{2}}-1-\log \left(\frac{\sigma^{2}}{\tilde{\sigma}^{2}}\right) \leq \frac{3}{4} t^{2} .
$$

Proof of Proposition 2. We first assume that $d=2$, and afterwards reduce the $d>2$ case to the $d=2$ case. The proof follows the standard reduction to binary hypothesis testing (specifically [105, Sec. 28.2.1]). We consider a pair of distributions $\mu_{ \pm}$for $Z$, and begin by showing that a single codebook and inverse covariance matrix pair cannot simultaneously achieve low $\mathbf{p}_{\mu}(C, S)-\inf _{C^{\prime}, S^{\prime}} \mathbf{p}_{\mu}\left(C^{\prime}, S^{\prime}\right)$ for both $\mu=\mu_{-}$and $\mu=\mu_{+}$.

Let $\eta \in\left(0, \frac{1}{4}\right)$ to be set later. Under $\mu_{+}\left(\right.$resp. $\left.\mu_{-}\right)$it is assumed that $Z \sim N\left(\mathbf{0}, \operatorname{diag}\left(\frac{1}{3 r_{s}}, \frac{1-2 \eta}{3 r_{s}}\right)\right)$ (resp. $\left.Z \sim N\left(\mathbf{0}, \operatorname{diag}\left(\frac{1-2 \eta}{3 r_{s}}, \frac{1}{3 r_{s}}\right)\right)\right)$. Given any $\left(x_{1}, x_{2}\right)$, if the Euclidean distance between $x_{1}$ and $x_{2}$ is increased, while the relative angle between them is preserved then the error probability decreases under both $\mu_{ \pm}$. Thus, $\inf _{C, S} \mathbf{p}_{\mu_{ \pm}}(C, S)$ is achieved with $x_{1}=\left(\cos \left(\alpha_{ \pm}\right), \sin \left(\alpha_{ \pm}\right)\right)=-x_{2}$ for some $\alpha_{ \pm}$, and similarly, it can be assumed that any optimal algorithm $A$ outputs an codebook of the form $x_{1}=\left(\cos \left(\alpha_{\boldsymbol{z}}\right), \sin \left(\alpha_{\boldsymbol{z}}\right)\right)=-x_{2}$ for some $\alpha_{\boldsymbol{z}}$. Now, note that $S$ can be parameterized by $(\beta, s)$ with $s(1) \equiv 1$ and $s(2) \equiv s$ in $(83)$. Now, it holds from symmetry that

$$
\inf _{C, S} \mathbf{p}_{\mu_{+}}(C, S)=\inf _{C, S} \mathbf{p}_{\mu_{-}}(C, S)
$$

Furthermore, under $\mu_{-}$, it is intuitively clear since the noise variance $\frac{1}{3 r_{s}}$ of the second dimension is larger than then one of the first dimension $\frac{1-2 \eta}{3 r_{s}}$, it is preferable to choose $x_{1}=x_{1}^{-}:=(1,0)$ and $x_{2}=x_{2}^{-}:=(-1,0)$, that is $\alpha_{-}=0$. Letting $C_{-}=\left(x_{1}^{-}, x_{2}^{-}\right)$we may bound 16

$$
\begin{aligned}
\inf _{C, S} \mathbf{p}_{\mu_{-}}(C, S) & \stackrel{(a)}{\leq} \inf _{S} \mathbf{p}_{\mu_{-}}\left(C_{-}, S\right) \\
& =\inf _{s \geq 1, \beta} \mathbf{p}_{\mu_{-}}\left(C_{-}, S\right) \\
& \stackrel{(b)}{=} \inf _{s \geq 1, \beta} Q\left(\frac{\cos ^{2}(\beta)+\sin ^{2}(\beta) s}{\sqrt{\frac{(1-2 \eta)}{3 r_{s}} \cdot\left[\cos ^{2}(\beta)+s \sin ^{2}(\beta)\right]^{2}+\frac{1}{3 r_{s}}[-\sin (\beta) \cos (\beta)+s \cos (\beta) \sin (\beta)]^{2}}}\right) \\
& \stackrel{(c)}{\leq} Q\left(\frac{1}{\sqrt{(1-2 \eta) /\left(3 r_{s}\right)}}\right)
\end{aligned}
$$

where $(a)$ follows from the choice $C=C_{-},(b)$ follows from Lemma 10 by setting $\alpha=\alpha_{-}=0, s(1)=1$, $s(2)=s, \sigma^{2}(1)=\frac{1-2 \eta}{3 r_{s}}$ and $\sigma^{2}(2)=\frac{1}{3 r_{s}}$, and $(c)$ follows from the choice $s=1$. Hence,

$$
\inf _{C, S} \mathbf{p}_{\mu_{-}}(C, S)=\inf _{C, S} \mathbf{p}_{\mu_{+}}(C, S) \leq Q\left(\frac{1}{\sqrt{(1-2 \eta) /\left(3 r_{s}\right)}}\right) .
$$

In addition, for an arbitrary fixed $(C, S)$ with $x_{1}=(\cos (\alpha), \sin (\alpha))=-x_{2}$, and $S$ parameterized by $(\beta, s)$, Lemma

\footnotetext{
${ }^{16}$ In fact, $C_{-}$is also an optimal choice, but this stronger property is not required for the bound.
} 
10, together with [110, further imply that

$$
\begin{aligned}
& \max _{\mu \in\left\{\mu_{ \pm}\right\}}\left[\mathbf{p}_{\mu}(C, S)-\inf _{C^{\prime}, S^{\prime}} \mathbf{p}_{\mu}\left(C^{\prime}, S^{\prime}\right)\right] \\
& \geq \frac{1}{2}\left[\mathbf{p}_{\mu_{-}}(C, S)-\inf _{C^{\prime}, S^{\prime}} \mathbf{p}_{\mu_{-}}\left(C^{\prime}, S^{\prime}\right)\right]+\frac{1}{2}\left[\mathbf{p}_{\mu_{+}}\left(C_{\boldsymbol{Z}}, S_{\boldsymbol{Z}}\right)-\inf _{C^{\prime}, S^{\prime}} \mathbf{p}_{\mu_{-}}\left(C^{\prime}, S^{\prime}\right)\right] \\
& =\frac{1}{2} Q\left(\frac{\cos ^{2}(\alpha+\beta)+\sin ^{2}(\alpha+\beta) s}{\sigma_{-}(\alpha, \beta, s)}\right)+\frac{1}{2} Q\left(\frac{\cos ^{2}(\alpha+\beta)+\sin ^{2}(\alpha+\beta) s}{\sigma_{+}(\alpha, \beta, s)}\right)-Q\left(\frac{1}{\sqrt{(1-2 \eta) /\left(3 r_{s}\right)}}\right)
\end{aligned}
$$

where

$$
\begin{aligned}
& \sigma_{-}^{2}(\alpha, \beta, s) \equiv \tau^{2}\left(\frac{1}{3 r_{s}}, \frac{1-2 \eta}{3 r_{s}}, \alpha, \beta, s\right), \\
& \sigma_{+}^{2}(\alpha, \beta, s) \equiv \tau^{2}\left(\frac{1-2 \eta}{3 r_{s}}, \frac{1}{3 r_{s}}, \alpha, \beta, s\right),
\end{aligned}
$$

with $\tau$ defined in (85). By Corollary 11, it is assured that the arguments of the three $Q$ functions in (112) are at least $\sqrt{3}$, and so Lemma 12 assures that $t \mapsto Q(1 / \sqrt{t})$ is convex. By that convexity property

$$
\begin{aligned}
& \frac{1}{2} Q\left(\frac{\cos ^{2}(\alpha+\beta)+\sin ^{2}(\alpha+\beta) s}{\sigma_{-}(\alpha, \beta, s)}\right)+\frac{1}{2} Q\left(\frac{\cos ^{2}(\alpha+\beta)+\sin ^{2}(\alpha+\beta) s}{\sigma_{+}(\alpha, \beta, s)}\right) \\
& \geq Q\left(\frac{\cos ^{2}(\alpha+\beta)+\sin ^{2}(\alpha+\beta) s}{\sqrt{\frac{1}{2} \sigma_{-}^{2}(\alpha, \beta, s)+\frac{1}{2} \sigma_{+}^{2}(\alpha, \beta, s)}}\right) \\
& =Q\left(\frac{\cos ^{2}(\alpha+\beta)+\sin ^{2}(\alpha+\beta) s}{\sigma(\alpha, \beta, s)}\right) \\
& \geq Q\left(\frac{1}{\sqrt{(1-\eta) /\left(3 r_{s}\right)}}\right)
\end{aligned}
$$

where

$$
\sigma^{2}(\alpha, \beta, s) \equiv \tau^{2}\left(\frac{1-\eta}{3 r_{s}}, \frac{1-\eta}{3 r_{s}}, \alpha, \beta, s\right)
$$

and the last inequality follows from the last statement of Lemma 10, Combining (112), (117), and the second property in Lemma 12 leads to

$$
\max _{\mu \in\left\{\mu_{ \pm}\right\}}\left[\mathbf{p}_{\mu}\left(C_{\boldsymbol{Z}}, S_{\boldsymbol{Z}}\right)-\inf _{C, S} \mathbf{p}_{\mu}(C, S)\right] \geq Q\left(\frac{1}{\sqrt{(1-\eta) /\left(3 r_{s}\right)}}\right)-Q\left(\frac{1}{\sqrt{(1-2 \eta) /\left(3 r_{s}\right)}}\right) \geq 0.145 \cdot \frac{\eta}{3 r_{s}} \equiv \epsilon
$$

where the last inequality holds since $\eta \in\left(0, \frac{1}{4}\right)$ and $r_{s} \leq 2$ is assumed. Hence, if we denote $L_{ \pm}:=\mathbf{p}_{\mu_{ \pm}}(C, S)-$ $\inf _{C^{\prime}, S^{\prime}} \mathbf{p}_{\mu_{ \pm}}\left(C^{\prime}, S^{\prime}\right)$ then the last display implies that if $L_{+}<\epsilon$ then $L_{-} \geq \epsilon$ and vice-versa. Consequently,

$$
\mathbb{1}\left\{L_{+} \geq \epsilon\right\}+\mathbb{1}\left\{L_{-} \geq \epsilon\right\}=\mathbb{1}\left\{L_{+}>\epsilon\right\}+1-\mathbb{1}\left\{L_{-}<\epsilon\right\}>1
$$

Thus, a single codebook cannot be simultaneously "good" for both $\mu_{ \pm}$. 
Now, let $A$ be an arbitrary algorithm which outputs $C_{\boldsymbol{z}}, S_{\boldsymbol{z}}$ with $C_{\boldsymbol{z}}$ of the form $x_{1}=\left(\cos \left(\alpha_{\boldsymbol{z}}\right), \sin \left(\alpha_{\boldsymbol{z}}\right)\right)=-x_{2}$ for some $\alpha_{\boldsymbol{z}}$, which can be assumed without loss of generality (w.l.o.g.) for an optimal algorithm. Denote by $f_{ \pm}(\boldsymbol{z})$ the density of $\mu_{ \pm}^{\otimes n}$, and denote $z_{i} \equiv\left(z_{i}(1), z_{i}(2)\right)$. Further denote the event $A:=\left\{\sum_{i=1}^{n} z_{i}^{2}(1) \geq \sum_{i=1}^{n} z_{i}^{2}(2)\right\}$ and its complement by $A^{c}$. Then,

$$
\begin{aligned}
& \sup _{\mu} \mathbb{P}_{\boldsymbol{Z} \sim \mu^{\otimes n}}\left[\mathbf{p}_{\mu}\left(C_{\boldsymbol{Z}}, S_{\boldsymbol{Z}}\right)-\inf _{C^{\prime}, S^{\prime}} \mathbf{p}_{\mu}\left(C^{\prime}, S^{\prime}\right)>\epsilon\right] \\
& \geq \max _{\mu \in\left\{\mu_{ \pm}\right\}} \mathbb{P}_{\boldsymbol{Z} \sim \mu}\left[\mathbf{p}_{\mu}\left(C_{\boldsymbol{Z}}, S_{\boldsymbol{Z}}\right)-\inf _{C^{\prime}, S^{\prime}} \mathbf{p}_{\mu}\left(C^{\prime}, S^{\prime}\right)>\epsilon\right] \\
& \stackrel{(a)}{\geq} \frac{1}{2} \mathbb{P}_{\boldsymbol{Z} \sim \mu_{-}^{\otimes n}}\left[L_{-}>\epsilon\right]+\frac{1}{2} \mathbb{P}_{\boldsymbol{Z} \sim \mu_{+}^{\otimes n}}\left[L_{+}>\epsilon\right] \\
& \stackrel{(b)}{=} \frac{1}{2} \int f_{-}(\boldsymbol{z}) \cdot \mathbb{1}\left[L_{-}>\epsilon\right] \mathbb{1}(A)+f_{+}(\boldsymbol{z}) \cdot \mathbb{1}\left[L_{+}>\epsilon\right] \mathbb{1}(A) \cdot \mathrm{d} \boldsymbol{z} \\
& +\frac{1}{2} \int f_{-}(\boldsymbol{z}) \cdot \mathbb{1}\left[L_{-}>\epsilon\right] \mathbb{1}\left(A^{c}\right)+f_{+}(\boldsymbol{z}) \cdot \mathbb{1}\left[L_{+}>\epsilon\right] \mathbb{1}\left(A^{c}\right) \cdot \mathrm{d} \boldsymbol{z} \\
& \stackrel{(c)}{\geq} \frac{1}{2} \int f_{-}(\boldsymbol{z}) \cdot\left(\mathbb{1}\left[L_{-}>\epsilon\right]+\mathbb{1}\left[L_{+}>\epsilon\right]\right) \mathbb{1}(A) \cdot \mathrm{d} \boldsymbol{z} \\
& +\frac{1}{2} \int f_{+}(\boldsymbol{z}) \cdot\left(\mathbb{1}\left[L_{-}>\epsilon\right]+\mathbb{1}\left[L_{+}>\epsilon\right]\right) \mathbb{1}\left(A^{c}\right) \cdot \mathrm{d} \boldsymbol{z} \\
& \stackrel{(d)}{\geq} \frac{1}{2} \int f_{-}(\boldsymbol{z}) \cdot \mathbb{1}(A) \cdot \mathrm{d} \boldsymbol{z}+\frac{1}{2} \int f_{+}(\boldsymbol{z}) \cdot \mathbb{1}\left(A^{c}\right) \cdot \mathrm{d} \boldsymbol{z} \\
& \stackrel{(e)}{=} \int f_{-}(\boldsymbol{z}) \cdot \mathbb{1}(A) \cdot \mathrm{d} \boldsymbol{z} \\
& =\mathbb{P}_{\boldsymbol{Z} \sim \mu_{-}^{\otimes n}}\left[\sum_{i=1}^{n} Z_{i}^{2}(1) \geq \sum_{i=1}^{n} Z_{i}^{2}(2)\right] \\
& \stackrel{(f)}{\geq} \frac{1}{4} \exp \left[-3 n \eta^{2}\right] \\
& \stackrel{(g)}{=} \delta \text {, }
\end{aligned}
$$

where $(a)$ utilizes the definition of $L_{ \pm},(b)$ utilizes the definition of the event $A$ and $f_{ \pm},(c)$ follows since $f_{+}(\boldsymbol{z})>$ $f_{-}(\boldsymbol{z})$ if and only if $\boldsymbol{z} \in A,(d)$ follows from 120$),(e)$ follows from symmetry, $(f)$ follows from Lemma 13 , and $(g)$ is obtained by setting $\eta=\sqrt{\frac{1}{3 n} \log \frac{1}{4 \delta}}$ while taking $n \geq n_{0}(\delta)$ such that $\eta<\frac{1}{4}$.

In case $d>2$, one may choose $\mu_{1}$ to be zero-mean Gaussian of covariance matrix $\operatorname{diag}\left(\frac{1}{3 r_{s}}, \frac{1-2 \eta}{3 r_{s}}, \tilde{\sigma}, \ldots, \tilde{\sigma}\right)$ (and similarly $\mu_{2}$ with the variances of the first two coordinates interchanged), and take an arbitrarily large value of $\tilde{\sigma}$ so the optimal algorithm will always choose the codewords to lie in the two-dimensional subspace spanned by the first two coordinates. The problem is then reduced to the $d=2$ case.

\section{The Proof of Theorem 3}

We will need several lemmas. The first lemma characterizes the continuity of the surrogate loss function w.r.t. $(C, S)$. 
Lemma 14. Suppose that $C=\left\{x_{j}\right\}_{j \in[m]} \in \mathcal{C}, \tilde{C}=\left\{\tilde{x}_{j}\right\}_{j \in[m]} \in \mathcal{C}$, and $S, \tilde{S} \in \mathcal{S}$ satisfy that there exists $\gamma_{x}, \gamma_{s} \geq 0$ such that $\left\|x_{j}-\tilde{x}_{j}\right\| \leq \gamma_{x}$ for all $j \in[m]$ and $\|S-\tilde{S}\|_{\mathrm{op}} \leq \gamma_{s}$. Then, for any $z \in \mathbb{B}^{d}(1)$

$$
|\bar{\ell}(C, S, z)-\bar{\ell}(\tilde{C}, \tilde{S}, z)| \leq \max _{j \in[m]}\left|\bar{\ell}_{j}(C, S, z)-\bar{\ell}_{j}(\tilde{C}, \tilde{S}, z)\right| \leq 8 \gamma_{x} r_{s}\left(r_{x}+1\right)+\gamma_{s}\left(\gamma_{x}^{2}+4 r_{x}\right) .
$$

Proof: For any $v, \tilde{v} \in \mathbb{R}^{d}$, it holds that

$$
\begin{aligned}
\left|v^{T} S z-\tilde{v}^{T} \tilde{S} z\right| & \leq\left|v^{T} S z-\tilde{v}^{T} S z\right|+\left|\tilde{v}^{T} S z-\tilde{v}^{T} \tilde{S} z\right| \\
& =\left|(v-\tilde{v})^{T} S z\right|+\left|\tilde{v}^{T}(S-\tilde{S}) z\right| \\
& \leq\|v-\tilde{v}\| \cdot\|S\|_{\mathrm{op}} \cdot\|z\|+\|\tilde{v}\| \cdot\|S-\tilde{S}\|_{\mathrm{op}} \cdot\|z\|,
\end{aligned}
$$

and

$$
\begin{aligned}
\left|\|v\|_{S}^{2}-\|\tilde{v}\|_{\tilde{S}}^{2}\right| & \leq\left|\|v\|_{S}^{2}-\|\tilde{v}\|_{S}^{2}\right|+\left|\|\tilde{v}\|_{S}^{2}-\|\tilde{v}\|_{\tilde{S}}^{2}\right| \\
& =\left|\left(S^{1 / 2} v\right)^{T}\left(S^{1 / 2} v\right)-\left(S^{1 / 2} \tilde{v}\right)^{T}\left(S^{1 / 2} \tilde{v}\right)\right|+\left|\tilde{v}^{T}(S-\tilde{S}) \tilde{v}\right| \\
& =\left|\left(S^{1 / 2} v+S^{1 / 2} \tilde{v}\right)^{T}\left(S^{1 / 2} v-S^{1 / 2} \tilde{v}\right)\right|+\left|\tilde{v}^{T}(S-\tilde{S}) \tilde{v}\right| \\
& \leq\|S\|_{\mathrm{op}} \cdot(\|v\|+\|\tilde{v}\|)\|v-\tilde{v}\|+\|\tilde{v}\|^{2} \cdot\|S-\tilde{S}\|_{\mathrm{op}},
\end{aligned}
$$

where $S^{1 / 2}$ is the symmetric square root of $S$. Setting $v=x_{j}-x_{j^{\prime}}$ and $\tilde{v}=\tilde{x}_{j}-\tilde{x}_{j^{\prime}}$ the last two displays and the triangle inequality imply that

$$
\left|\left(x_{j}-x_{j^{\prime}}\right)^{T} S z-\left(\tilde{x}_{j}-\tilde{x}_{j^{\prime}}\right)^{T} \tilde{S} z\right| \leq 2 \gamma_{x} r_{s}+2 r_{x} \gamma_{s}
$$

and

$$
\left|\left\|x_{j}-x_{j^{\prime}}\right\|_{S}^{2}-\left\|\tilde{x}_{j}-\tilde{x}_{j^{\prime}}\right\|_{\tilde{S}}^{2}\right| \leq 8 r_{x} \gamma_{x} r_{s}+\gamma_{x}^{2} \gamma_{s}
$$

for all $j, j^{\prime} \in[m]$. We may now peel the difference between the loss functions. Let us denote

$$
a_{j, j^{\prime}}(C, S, z):=\left(\left\|x_{j}-x_{j^{\prime}}\right\|_{S}^{2}+2\left(x_{j}-x_{j^{\prime}}\right)^{T} S z\right) .
$$

Then,

$$
\begin{aligned}
\left|\bar{\ell}_{j}(C, S, z)-\bar{\ell}_{j}(\tilde{C}, \tilde{S}, z)\right| & \stackrel{(a)}{\leq}\left|\min _{j^{\prime} \in[m] \backslash\{j\}} a_{j, j^{\prime}}(C, S, z)-\min _{j^{\prime} \in[m] \backslash\{j\}} a_{j, j^{\prime}}(\tilde{C}, \tilde{S}, z)\right| \\
& \stackrel{(b)}{\leq} \max _{j^{\prime} \in[m] \backslash\{j\}}\left|a_{j, j^{\prime}}(C, S, z)-a_{j, j^{\prime}}(\tilde{C}, \tilde{S}, z)\right| \\
& \leq \max _{j^{\prime} \in[m] \backslash\{j\}}\left\{\left|\left\|x_{j}-x_{j^{\prime}}\right\|_{S}^{2}-\left\|\tilde{x}_{j}-\tilde{x}_{j^{\prime}}\right\|_{\tilde{S}}^{2}\right|+\left|2\left(\tilde{x}_{j}-\tilde{x}_{j^{\prime}}\right)^{T} \tilde{S} z-2\left(x_{j}-x_{j^{\prime}}\right)^{T} S z\right|\right\} \\
& \stackrel{(c)}{\leq} 8 \gamma_{x} r_{s}\left(r_{x}+1\right)+\gamma_{s}\left(\gamma_{x}^{2}+4 r_{x}\right)
\end{aligned}
$$


where $(a)$ holds since $t \mapsto[1-t]_{+}$is a 1-Lipschitz function, $(b)$ holds since for any $\left\{\alpha_{j, j^{\prime}}\right\},\left\{\tilde{\alpha}_{j, j^{\prime}}\right\}$

$$
\left|\min _{j^{\prime} \in[m] \backslash\{j\}} \alpha_{j, j^{\prime}}-\min _{j^{\prime} \in[m] \backslash\{j\}} \tilde{\alpha}_{j, j^{\prime}}\right| \leq \max _{j^{\prime} \in[m] \backslash\{j\}}\left|\alpha_{j, j^{\prime}}-\tilde{\alpha}_{j, j^{\prime}}\right|,
$$

and $(c)$ holds by utilizing (138) and (139).

We denote by $\mathrm{N}\left(\mathcal{S},\|\cdot\|_{\mathrm{op}}, \gamma_{s}\right)$ the covering number (e.g. [118, Definition 4.2.2]) of $\mathcal{S}$, for the operator norm and covering radius $\gamma_{s}$.

Lemma 15. It holds that

$$
\mathrm{N}\left(\mathcal{S},\|\cdot\|_{\mathrm{op}}, \gamma_{s}\right) \leq 2\left[\frac{12 d r_{s}}{\gamma_{s}}\right]^{d(d+1)} .
$$

Proof: Denote the eigendecomposition of $S \in \mathbb{S}_{+}^{d}$ by $S=U \Lambda U^{T}$, where $U \in \mathbb{R}^{d \times d}$ is an orthonormal matrix and $\Lambda \in \mathbb{R}_{+}^{d \times d}$ is diagonal. Further let $u_{i}$ be the $i$ th eigenvector of $S$ (i.e., the $i$ th column of $U$ ), and $\lambda_{i} \in\left[0, r_{s}\right]$ be the $i$ th eigenvalue (i.e., the $(i, i)$ element of $\Lambda$ ). Then, using analogous notation for $\tilde{S} \in \mathbb{S}_{+}^{d}$,

$$
\begin{aligned}
\|S-\tilde{S}\|_{\mathrm{op}} & =\left\|U \Lambda U^{T}-\tilde{U} \tilde{\Lambda} \tilde{U}^{T}\right\|_{\mathrm{op}} \\
& \leq\left\|U \Lambda U^{T}-U \tilde{\Lambda} U^{T}\right\|_{\mathrm{op}}+\left\|U \tilde{\Lambda} U^{T}-\tilde{U} \tilde{\Lambda} \tilde{U}^{T}\right\|_{\mathrm{op}} \\
& \leq \max _{i \in[d]}\left|\lambda_{i}-\tilde{\lambda}_{i}\right|+2 d \max _{i \in[d]} \tilde{\lambda}_{i}\left\|u_{i}-\tilde{u}_{i}\right\|,
\end{aligned}
$$

where in (149): The first term is bounded as

$$
\left\|U \Lambda U^{T}-U \tilde{\Lambda} U^{T}\right\|_{\mathrm{op}} \leq\|\Lambda-\tilde{\Lambda}\|_{\mathrm{op}}=\max _{i \in[d]}\left|\lambda_{i}-\tilde{\lambda}_{i}\right|
$$

The second term is bounded as

$$
\left\|U \tilde{\Lambda} U^{T}-\tilde{U} \tilde{\Lambda} \tilde{U}^{T}\right\|_{\mathrm{op}}=\left\|\sum_{i=1}^{d} \tilde{\lambda}_{i}\left(u_{i} u_{i}^{T}-\tilde{u}_{i} \tilde{u}_{i}^{T}\right)\right\|_{\mathrm{op}} \leq \sum_{i=1}^{d} \tilde{\lambda}_{i}\left\|\left(u_{i} u_{i}^{T}-\tilde{u}_{i} \tilde{u}_{i}^{T}\right)\right\|_{\mathrm{op}} \leq \sum_{i=1}^{d} 2 \tilde{\lambda}_{i}\left\|u_{i}-\tilde{u}_{i}\right\|
$$

since for any $v \in \mathbb{S}^{d-1}$

$$
\left|v^{T}\left(u_{i} u_{i}^{T}-\tilde{u}_{i} \tilde{u}_{i}^{T}\right) v\right|=\left|u_{i}^{T} v v^{T} u_{i}-\tilde{u}_{i}^{T} v v^{T} \tilde{u}_{i}\right|=\left|\left\|u_{i}\right\|_{v v^{T}}^{2}-\left\|\tilde{u}_{i}\right\|_{v v^{T}}^{2}\right| \leq 2\left\|u_{i}-\tilde{u}_{i}\right\|
$$

with the last inequality follows as in 137 . Now, let $\epsilon=\frac{\gamma_{s}}{4 d r_{s}}$ and let $\mathcal{U}$ be an $\epsilon$-net in the Euclidean distance for the unit sphere $\mathbb{S}^{d-1}$ whose size is less than $|\mathcal{C}| \leq\left(\frac{3}{\epsilon}\right)^{d}$ (whose existence is assured from [118, Corollary 4.2.13]). Further, let $\epsilon_{0}=\frac{\gamma_{s}}{2}$, and let $\mathcal{L}$ be a proper $\epsilon_{0}$-net in the in the $\ell_{1}$ norm for $\left[0, r_{s}\right]$ whose size is $|\mathcal{L}| \leq\left(\frac{r_{s}}{\epsilon_{0}}\right)$. By (149), the set

$$
\left\{U \Lambda U^{T}: U^{T} U=I_{d}, U=\left[u_{1}, \ldots, u_{d}\right], \Lambda=\operatorname{diag}\left(\lambda_{1}, \ldots, \lambda_{d}\right), u_{i} \in \mathcal{U} \subset \mathbb{R}^{d}, \lambda_{i} \in \mathcal{L}, \forall i \in[d]\right\}
$$

is a $\gamma_{s}$-cover of $\mathcal{S}$ whose size is $(|\mathcal{U}| \cdot|\mathcal{L}|)^{d}$ (where the $d$ th power follows from allowing an independent choice for $i \in[d]$ ), which is less the r.h.s. of (146). 
We say that $\mathcal{K} \subset \mathbb{R}^{n}$ is a $\gamma$-cover of $\mathcal{L} \subset \mathbb{R}^{n}$ under the metric $\|\cdot\|_{p}, p \in[1, \infty]$, if

$$
\sup _{l^{n} \in \mathcal{L}} \min _{k^{n} \in \mathcal{K}} \frac{1}{n} \sum_{i=1}^{n}\left|l_{i}-k_{i}\right|^{p} \leq \gamma^{p},
$$

where $l^{n}=\left(l_{1}, \ldots, l_{n}\right)$ and $k^{n}=\left(k_{1}, \ldots, k_{n}\right)$. The $\gamma$-covering number of $\mathcal{L} \subset \mathbb{R}^{n}$ under the metric $\|\cdot\|_{p}$ is denoted by

$$
\mathrm{N}_{p}(\gamma, \mathcal{L}):=\min \{|\mathcal{K}|: \mathcal{K} \text { is a } \gamma \text {-cover of } \mathcal{L}\}
$$

The next bound on the empirical Rademacher complexity (66) is well-known (e.g., [97, Thm. 12.4]).

Lemma 16 (Dudley's entropy integral). For $\mathcal{L} \subset \mathbb{R}^{n}$

$$
\operatorname{Rad}(\mathcal{L}) \leq \inf _{\alpha \geq 0}\left\{4 \alpha+\frac{12}{\sqrt{n}} \int_{\alpha}^{1} \sqrt{\log \mathrm{N}_{2}(\gamma, \mathcal{L})} \cdot \mathrm{d} \gamma\right\}
$$

With this bound we may obtain the following bound on the empirical Rademacher complexity for the loss class induced by the surrogate error probability loss function:

Lemma 17. Let $\boldsymbol{z}=\left(z_{1}, \ldots, z_{n}\right) \in \mathbb{R}^{n}$ be given, and consider the loss class

$$
\overline{\mathcal{L}}_{\boldsymbol{z}}:=\left\{\bar{\ell}(C, S, \boldsymbol{z}) \in \mathbb{R}_{+}^{n}: C \in \mathcal{C}, S \in \mathcal{S}\right\} \subset \mathbb{R}_{+}^{n},
$$

where $\bar{\ell}(C, S, \boldsymbol{z})=\left(\bar{\ell}\left(C, S, z_{1}\right), \ldots \bar{\ell}\left(C, S, z_{n}\right)\right)$. Then, assuming $r_{x} \geq 1$ and $r_{s} \geq 1$ :

$$
\operatorname{Rad}\left(\overline{\mathcal{L}}_{\boldsymbol{z}}\right) \leq 28 \sqrt{\frac{(d \vee m)(d+1) \log \left(31 \cdot d r_{s} r_{x}\right)}{n}} .
$$

Proof: We will bound the Rademacher complexity of the loss class induced by the surrogate loss function in 157, using covering arguments and Dudley's entropy integral. Assume that $\gamma_{x} \in[0,1]$. Let $\tilde{\mathcal{C}}_{1}$ be a $\gamma_{x}$-net of $\mathcal{C}_{1}$ in the Euclidean norm whose size is less than $\left|\tilde{\mathcal{C}}_{1}\right| \leq\left(\frac{3 r_{x}}{\gamma_{x}}\right)^{d}$, and whose existence is assured from [118. Corollary 4.2.13], and also let $\tilde{\mathcal{C}}=\left(\tilde{\mathcal{C}}_{1}\right)^{m}$. In addition, let $\tilde{\mathcal{S}}$ be a $\gamma_{s}$-net of $\mathcal{S}$ in the operator norm whose size is less than $|\tilde{\mathcal{S}}| \leq 2\left(\frac{12 d r_{s}}{\gamma_{s}}\right)^{d(d+1)}$, whose existence is assured from Lemma 15 . Then, by Lemma 14 , the set

$$
\tilde{\mathcal{L}}_{\boldsymbol{z}}:=\left\{\bar{\ell}(C, S, \boldsymbol{z}) \in \mathbb{R}_{+}^{n}: C \in \tilde{\mathcal{C}}, S \in \tilde{\mathcal{S}}\right\}
$$

is a $\gamma$-cover of $\overline{\mathcal{L}}_{\boldsymbol{z}}$ with

$$
\gamma=8 \gamma_{x} r_{s}\left(r_{x}+1\right)+\gamma_{s}\left(\gamma_{x}^{2}+4 r_{x}\right)
$$

whose size is less than

$$
2\left[\frac{12 d\left(r_{s}+1\right)}{\gamma_{s}}\right]^{d(d+1)} \cdot\left(\frac{3 r_{x}}{\gamma_{x}}\right)^{m(d+1)} \leq\left[\frac{72 \cdot d \cdot r_{s} r_{x}}{\gamma_{s} \gamma_{x}}\right]^{(d \vee m)(d+1)}
$$

We may next optimize over $\left(\gamma_{x}, \gamma_{s}\right)$ to achieve the minimal covering size for any given $\gamma$, or alternatively, to minimize $\gamma$ in (160) under the size constraint defined by $\psi:=\gamma_{x} \gamma_{s}>0$, where we assume that $\psi<1$. Substituting 
$\gamma_{s}=\psi / \gamma_{x}$ in 160 we obtain

$$
\begin{aligned}
\gamma & \leq \min _{\gamma_{x}>0}\left\{\gamma_{x}\left(8 r_{s}\left(r_{x}+1\right)+\psi\right)+\frac{4 r_{x} \psi}{\gamma_{x}}\right\} \\
& =2 \sqrt{\left(8 r_{s}\left(r_{x}+1\right)+\psi\right) 4 r_{x} \psi} \\
& \leq 20 r_{s} r_{x} \sqrt{\psi}
\end{aligned}
$$

where the equality follows since the minimizer of $a \gamma_{x}+b / \gamma_{x}$ over $\gamma_{x} \geq 0$ for $a, b \in \mathbb{R}_{+}$is at $\gamma_{x}=\sqrt{b / a}$ and the minimal value is $2 \sqrt{a b}$, and the (generous) inequality using the assumptions $r_{x} \geq 1, r_{s} \geq 1$, and $\psi<1$. Hence, under these assumptions,

$$
\psi=\gamma_{x} \gamma_{s} \geq \frac{\gamma^{2}}{400 r_{s}^{2} r_{x}^{2}}
$$

Thus, for a given $\gamma>0$, the logarithm of the cover size is upper bounded as

$$
(d \vee m)(d+1) \log \left[\frac{28800 \cdot d r_{s}^{3} r_{x}^{3}}{\gamma^{2}}\right]=a-2(d \vee m)(d+1) \log \gamma
$$

where $a:=(d \vee m)(d+1) \log \left(28800 \cdot d r_{s}^{3} r_{x}^{3}\right)$. By Lemma 16

$$
\begin{aligned}
\operatorname{Rad}\left(\overline{\mathcal{L}}_{\boldsymbol{z}}\right) & \leq \lim _{\alpha \rightarrow 0} \frac{12}{\sqrt{n}} \int_{\alpha}^{1} \sqrt{a-2(d \vee m)(d+1) \log \gamma} \cdot \mathrm{d} \gamma \\
& \leq 12 \sqrt{\frac{a}{n}} \lim _{\alpha \rightarrow 0} \int_{\alpha}^{1} 1-\frac{2(d \vee m)(d+1) \log \gamma}{2 a} \cdot \mathrm{d} \gamma \\
& =12 \sqrt{\frac{a}{n}}\left[1+\frac{(d \vee m)(d+1)}{a}\right]
\end{aligned}
$$

where the second inequality follows from $\sqrt{1-t} \leq 1-t / 2$ for $t \in(-\infty, 1]$, and the following equality using a continuity argument implied by $\lim _{t \rightarrow 0} t \cdot \log t=0$. The result follows by inserting back the definition of $a$ and generously bounding using $r_{x} \geq 1$ and $r_{s} \geq 1$, and then simplifying.

The proof of Theorem 3 follows immediately:

Proof of Theorem 3. We use Prop. 8 and note that

$$
\begin{aligned}
\left|\bar{\ell}_{j}(C, S, z)\right| & \leq 1 \vee \max _{j^{\prime} \in[m], j^{\prime} \neq j}\left(\left\|x_{j}-x_{j^{\prime}}\right\|_{S}^{2}+\left|2\left(x_{j}-x_{j^{\prime}}\right)^{T} S z\right|\right) \\
& \leq 1 \vee\left\{4 r_{x}^{2} r_{s}+4 r_{x} r_{s}\right\}=: r
\end{aligned}
$$

Bounding the Rademacher complexity using Lemma 17 completes the proof.

D. The Proofs of Theorems 4 and 5

We will utilize the following lemma regarding the KL divergence.

Lemma 18. Let $Q$ be a measure and let $l, \tilde{l}: \mathcal{X} \mapsto \mathbb{R}$ be measurable functions on a measurable space $\mathcal{X}$. Further let $P(\mathrm{~d} x) \propto Q(\mathrm{~d} x) \cdot e^{-\beta l(x)}$ (resp. $\left.\tilde{P}(\mathrm{~d} x) \propto Q(\mathrm{~d} x) \cdot e^{-\beta \tilde{l}(x)}\right)$ be the Gibbs measures based on the loss functions $l$ (resp. $\tilde{l})$. If $\|l-\tilde{l}\|_{\infty} \leq \epsilon$ then $\mathrm{d}_{\mathrm{KL}}(P \| \tilde{P}) \leq 2 \beta \epsilon \cdot\left\{1 \wedge\left(e^{2 \beta \epsilon}-1\right)\right\}$. 
Proof: For any $f, g: \mathcal{X} \mapsto \mathbb{R}^{+}$it holds that $\frac{\mathbb{E}_{Q}[f]}{\mathbb{E}_{Q}[g]} \leq\left\|\frac{f}{g}\right\|_{\infty}$ where $\|\cdot\|_{\infty}$ is the sup norm. Hence,

$$
\left|\log \frac{P(\mathrm{~d} x)}{\tilde{P}(\mathrm{~d} x)}\right|=\log \left\{\frac{e^{-\beta l(x)}}{e^{-\beta \tilde{l}(x)}} \cdot \frac{\mathbb{E}_{Q}\left[e^{-\beta \tilde{l}(x)}\right]}{\mathbb{E}_{Q}\left[e^{-\beta l(x)}\right]}\right\} \leq 2 \beta \epsilon,
$$

and so it holds that $\mathrm{d}_{\mathrm{KL}}(P \| \tilde{P})=\mathbb{E}_{P}\left[\log \frac{P(\mathrm{~d} x)}{\tilde{P}(\mathrm{~d} x)}\right] \leq 2 \beta \epsilon$. Furthermore, by [27, Lemma 3.18], it also holds that $\mathrm{d}_{\mathrm{KL}}(P \| \tilde{P}) \leq 2 \beta \epsilon \cdot\left(e^{2 \beta \epsilon}-1\right)$.

Proof of empirical error bound of Theorem $4-$ - 33): Let $\mathrm{d}_{\mathrm{TV}}(\mu \| \tilde{\mu})$ denote the total variation distance between the probability measures $\mu$ and $\tilde{\mu}$. By the variational representation of the total variation and Pinsker's inequality [112, Lemma 2.5], for any two measures $\mu, \tilde{\mu}$ and a function $f: \mathcal{X} \mapsto \mathbb{R}$ with $\|f\|_{\infty} \leq 1 / 2$ it holds that

$$
\left|\mathbb{E}_{X \sim \mu}[f(X)]-\mathbb{E}_{X \sim \tilde{\mu}}[f(X)]\right| \leq \mathrm{d}_{\mathrm{TV}}(\mu \| \tilde{\mu}) \leq \sqrt{\frac{\mathrm{d}_{\mathrm{KL}}(\mu \| \tilde{\mu})}{2 \log e}} .
$$

We further denote by $P_{C_{\mu, 1} \cdots C_{\mu, T}}$ (resp. $P_{C_{z, 1} \cdots C_{z, T}}$ ) the joint probability measure of $\boldsymbol{C}_{\mu}$ (resp. $\boldsymbol{C}_{\boldsymbol{Z}}$ ), and use standard notation for their conditional versions. Furthermore, we let $\mathfrak{C}$ be the set of all possibly expurgated codebooks over all $T$ steps, i.e., the initial codebook $C_{0}$, and each of the $\left(\begin{array}{c}m_{0} \\ m_{0}-k t\end{array}\right)$ codebooks at the th stage, for any $t \in[T]$. There is a total of

$$
1+\left(\begin{array}{c}
m_{0} \\
m_{0}-k
\end{array}\right)+\left(\begin{array}{c}
m_{0} \\
m_{0}-2 k
\end{array}\right)+\cdots\left(\begin{array}{c}
m_{0} \\
m
\end{array}\right) \stackrel{(*)}{\leq} T\left(\begin{array}{c}
m_{0} \\
m_{0} / 2
\end{array}\right) \leq \frac{m_{0}^{m_{0} / 2+1}}{k}:=\alpha_{m_{0}, k}
$$

such different codebooks, where $(*)$ follows from the assumption $m_{0} \geq 2 m$. For any $\delta \in(0,1)$, define $\epsilon=$ $\sqrt{\frac{1}{2 n} \log \frac{\alpha_{m_{0}, k}}{\delta}}$ and the event

$$
E:=\left\{z: \sup _{C \in \mathfrak{C}}\left|\mathbf{p}_{\boldsymbol{z}}(C)-\mathbf{p}_{\mu}(C)\right|<\epsilon\right\}
$$

Hoeffding's inequality and the union bound then imply that $\mathbb{P}[E] \geq 1-\delta$. For $z \in E$, the definitions of the Gibbs algorithms (population and empirical versions) and Lemma 18 imply that for any $t \in[T]$ and $C \in \mathfrak{C}$

$$
\mathrm{d}_{\mathrm{KL}}\left(P_{C_{\boldsymbol{z}, t} \mid C_{\boldsymbol{z}, t-1}}(\cdot \mid C)|| P_{C_{\mu, t} \mid C_{\mu, t-1}}(\cdot \mid C)\right) \leq 2 \beta \epsilon \cdot\left(1 \wedge\left(e^{2 \beta \epsilon}-1\right)\right)
$$

and for $\boldsymbol{z} \in E^{c}$ and any $C \subset\left(\mathbb{R}^{d}\right)^{m_{t-1}}$

$$
\mathrm{d}_{\mathrm{KL}}\left(P_{C_{\boldsymbol{z}, t} \mid C_{\boldsymbol{z}, t-1}}(\cdot \mid C)|| P_{C_{\mu, t} \mid C_{\mu, t-1}}(\cdot \mid C)\right) \leq 2 \beta \cdot\left(1 \wedge\left(e^{2 \beta}-1\right)\right) .
$$

Condition on any given $\boldsymbol{Z}=\boldsymbol{z} \in E$ and $C_{0}$ (implicitly) and averaging over the randomness of the Gibbs algorithm

$$
\begin{aligned}
\left|\mathbb{E}\left[\mathbf{p}_{\mu}\left(C_{\boldsymbol{z}, T}\right)-\mathbf{p}_{\mu}\left(C_{\mu, T}\right) \mid \boldsymbol{Z}=\boldsymbol{z}\right]\right| & \stackrel{(a)}{\leq} \sqrt{\frac{\mathrm{d}_{\mathrm{KL}}\left(P_{C_{\boldsymbol{z}, T}}|| P_{C_{\mu, T}}\right)}{2 \log e}} \\
& \leq \sqrt{\frac{\mathrm{d}_{\mathrm{KL}}\left(P_{C_{\boldsymbol{z}, 1} \cdots C_{\boldsymbol{z}, T}} \| P_{C_{\mu, 1} \cdots C_{\mu, T}}\right)}{2 \log e}} \\
& \stackrel{(b)}{=} \sqrt{\frac{\sum_{t=0}^{T-1} \mathbb{E}\left[\mathrm{d}_{\mathrm{KL}}\left(P_{C_{\boldsymbol{z}, t} \mid C_{\boldsymbol{z}, t-1}}\left(\cdot \mid C_{\boldsymbol{z}, t-1}\right)|| P_{C_{\mu, t} \mid C_{\mu, t-1}}\left(\cdot \mid C_{\boldsymbol{z}, t-1}\right)\right)\right]}{2 \log e}}
\end{aligned}
$$




$$
\stackrel{(c)}{\leq} \sqrt{\frac{2 T \beta \epsilon \cdot\left(1 \wedge\left(e^{2 \beta \epsilon}-1\right)\right)}{2 \log e}},
$$

where $(a)$ follows from 173 (which holds since $\mathbf{p}_{\mu}(C) \in[0,1]$ ), (b) follows from the chain rule and the Markov property $\left(P_{C_{z, 1} \cdots C_{z, T}}=P_{C_{z, 1}} \otimes P_{C_{z, 2} \mid C_{z, 1}} \cdots \otimes P_{C_{z, T} \mid C_{z, T-1}}\right)$ of $\boldsymbol{C}_{\boldsymbol{z}}$ conditioned on $\boldsymbol{z}$ and a similar property which holds for $C_{\mu}$, and $(c)$ follows from utilizing (176). A similar bound holds for $z \in E^{c}$ with $\epsilon$ replaced by 1 . Then,

$$
\begin{aligned}
\mathbb{E}\left[\mathbf{p}_{\mu}\left(C_{\boldsymbol{z}, T}\right)-\mathbf{p}_{\mu}\left(C_{\mu, T}\right)\right]= & \mathbb{P}[\boldsymbol{Z} \in E] \cdot \mathbb{E}\left[\left(\mathbf{p}_{\mu}\left(C_{\boldsymbol{z}, T}\right)-\mathbf{p}_{\mu}\left(C_{\mu, T}\right)\right) \mid \boldsymbol{Z} \in E\right] \\
& +\mathbb{P}\left[\boldsymbol{Z} \in E^{c}\right] \cdot \mathbb{E}\left[\left(\mathbf{p}_{\mu}\left(C_{\boldsymbol{z}, T}\right)-\mathbf{p}_{\mu}\left(C_{\mu, T}\right)\right) \mid \boldsymbol{Z} \in E^{c}\right] \\
\leq & \sqrt{\frac{T \beta}{\log e}} \cdot\left(\sqrt{\epsilon\left(e^{2 \beta \epsilon}-1\right)}+\delta\right) .
\end{aligned}
$$

The proof of the bound is completed by choosing $\delta=\frac{1}{n}$, the assumption in 32 implies that $2 \beta \epsilon \leq 1$ and using $e^{x}-1 \leq(e-1) x$ for $x \in[0,1]$ we obtain

$$
\begin{aligned}
\mathbb{E}\left[\mathbf{p}_{\mu}\left(C_{\boldsymbol{z}, T}\right)-\mathbf{p}_{\mu}\left(C_{\mu, T}\right)\right] & \leq \sqrt{\frac{T \beta}{\log e}} \cdot\left(\sqrt{(e-1) \beta \frac{\log \left(n \alpha_{m_{0}, k}\right)}{n}}+\frac{1}{n}\right) \\
& \leq \sqrt{\frac{4(e-1)}{\log e} \cdot \frac{T \beta^{2} \log \left(n \alpha_{m_{0}, k}\right)}{n}} .
\end{aligned}
$$

We next prove the bounds on the average generalization error. To this end we note that a randomized learning algorithm $A: Z \mapsto \mathcal{A}$ is conditional probability distribution $P_{A \mid Z}$, where $\mathcal{A}$ is the set of possible outputs which is assumed to be a measurable space. As was shown in [94], [95], [128], the generalization error of learning algorithms can be controlled by the mutual information between the output hypothesis $A \in \mathcal{A}$ and the input data, and such a bound is used here.

Proof of generalization error bound of Theorem 4- (34): For brevity, let $C_{\boldsymbol{Z}, t}$ be denoted here as $C_{t}$. We use standard information-theoretic notation [17] for entropy and mutual information. Recall that the erasure mutual information [117] between $U$ and $\boldsymbol{V}=\left(V_{1}, \ldots, V_{n}\right)$ is defined as

$$
I^{-}(U ; \boldsymbol{V}):=\sum_{i=1}^{n} I\left(U ; V_{i} \mid \boldsymbol{V}^{-i}\right)
$$

where $\boldsymbol{V}^{-i}=\left(V_{1}, \ldots, V_{i-1}, V_{i+1}, \ldots, V_{n}\right)$. To bound the generalization error we analyze the information-theoretic stability properties of the Gibbs algorithm, and specifically bound the mutual information $I\left(C_{T} ; \boldsymbol{Z}\right)$. First note the the expurgation algorithm can be described as an adaptive composition of algorithms. Specifically, at step $t$, the output of the algorithm $C_{t}$ is a function of $C_{t-1}$ and $\boldsymbol{Z}$ and so the Markov relation $\left(C_{1}, \ldots, C_{t-1}\right)-\left(C_{t}, \boldsymbol{Z}\right)-C_{t+1}$ holds. We then have

$$
I\left(C_{T} ; \boldsymbol{Z}\right) \stackrel{(a)}{\leq} I^{-}\left(C_{T} ; \boldsymbol{Z}\right)
$$




$$
\begin{aligned}
& =\sum_{i=1}^{n} I\left(C_{T} ; Z_{i} \mid \boldsymbol{Z}^{-i}\right) \\
& \stackrel{(b)}{\leq} \sum_{i=1}^{n} I\left(C_{1}, \ldots, C_{T} ; Z_{i} \mid \boldsymbol{Z}^{-i}\right) \\
& =\sum_{i=1}^{n} \sum_{t=1}^{T} I\left(C_{t} ; Z_{i} \mid \boldsymbol{Z}^{-i}, C_{t-1}, \ldots, C_{1}\right) \\
& =\sum_{i=1}^{n} \sum_{t=1}^{T} H\left(C_{t} \mid \boldsymbol{Z}^{-i}, C_{t-1}, \ldots, C_{1}\right)-H\left(C_{t} \mid \boldsymbol{Z}, C_{t-1}, \ldots, C_{1}\right) \\
& \stackrel{(c)}{\leq} \sum_{i=1}^{n} \sum_{t=1}^{T} H\left(C_{t} \mid \boldsymbol{Z}^{-i}, C_{t-1}\right)-H\left(C_{t} \mid \boldsymbol{Z}, C_{t-1}\right) \\
& =\sum_{t=1}^{T} I^{-}\left(C_{t} ; \boldsymbol{Z} \mid C_{t-1}\right)
\end{aligned}
$$

where (a) follows since $Z$ is comprised of $n$ independent random variables and [95, Prop. 1], (b) follows from the chain rule and non-negativity of mutual information, and $(c)$ follows from the Markov property and since conditioning reduces entropy. We thus upper bound the erasure mutual information $I^{-}\left(C_{t} ; \boldsymbol{Z} \mid C_{t-1}\right)$. As was shown in the proof of Lemma 18 , for any $\boldsymbol{z}, \boldsymbol{z}^{\prime} \in\left(\mathbb{R}^{d}\right)^{n}$ such that $\mathrm{d}_{\mathrm{H}}\left(\boldsymbol{z}, \boldsymbol{z}^{\prime}\right)=1$,

$$
\left|\log \frac{\mathbb{P}\left[C_{t+1} \mid \boldsymbol{Z}, C_{t}\right]}{\mathbb{P}\left[C_{t+1} \mid \boldsymbol{Z}^{\prime}, C_{t}\right]}\right| \leq \frac{2 \beta}{n}
$$

Derivation similar to the proof of [94, Thm. 4] (see also [95, Thm. 7]) then implies that $I^{-}\left(C_{t} ; \boldsymbol{Z} \mid C_{t-1}\right) \leq 2 \beta \wedge \frac{\beta^{2}}{2 n}$. As this holds for all $t \in[T], 193$ thus implies that $I\left(C_{T} ; \boldsymbol{Z}\right) \leq T \cdot\left(2 \beta \wedge \frac{\beta^{2}}{2 n}\right)$ and the Gibbs algorithm is stable in the mutual information. Since the loss function is the error probability that is bounded to $[0,1]$ and so is $\frac{1}{4}$-sub-Gaussian, it follows from [128, Thm. 1] that

$$
\mathbb{E}\left[\mathbf{p}_{\mu}\left(C_{\boldsymbol{Z}, T}\right)-\mathbf{p}_{\boldsymbol{Z}}\left(C_{\boldsymbol{Z}, T}\right)\right] \leq \sqrt{T\left(\frac{\beta}{n} \wedge \frac{\beta^{2}}{4 n^{2}}\right)} .
$$

To prove the high probability bound of Theorem 5 we recall that a learning algorithm is termed $(\varepsilon, \eta)$-differentiallyprivate [27] if for any measurable set $F \subseteq \mathcal{A}$

$$
\mathrm{d}_{\mathrm{H}}\left(\boldsymbol{z}, \boldsymbol{z}^{\prime}\right) \leq 1 \Rightarrow P_{A \mid \boldsymbol{Z}=\boldsymbol{z}}(F) \leq e^{\varepsilon} P_{A \mid \boldsymbol{Z}=\boldsymbol{z}^{\prime}}(F)+\eta
$$

Let $C_{0} \subset \mathcal{C}$ be a super-codebook of size $m_{0}$, and let $\mathcal{A}_{t}$ be the set of its subsets of size $m_{t}=m_{0}-k t$, and $T=\frac{m_{0}-m}{k}$ for some given $k$ (assuming $T$ is integer). For brevity, we suppress $C_{0}$ from the notation, as it is assumed that $C_{0}$ is fixed in advance, and in accordance, the high probability bound of Theorem 5 only refers to the random draw of the noise samples $Z$. The Gibbs algorithm $C_{\mathrm{G}}:\left(\mathbb{R}^{d}\right)^{n} \mapsto \mathcal{A}_{T}$ is defined by the sequence of 
algorithms $\left\{C_{t}\right\}_{t=1}^{T}$ in 29, , as

$$
P_{C_{\mathrm{G}} \mid \boldsymbol{z}, C_{0}}=P_{C_{1} \mid \boldsymbol{z}} \otimes P_{C_{2} \mid C_{1}, \boldsymbol{z}} \otimes P_{C_{3} \mid C_{2}, \boldsymbol{z}} \otimes \cdots \otimes P_{C_{T} \mid C_{T-1}, \boldsymbol{z}}
$$

Lemma 19. $C_{G}$ is a $\left(\frac{T \beta}{n}, 0\right)$-differentially private algorithm. Furthermore, if $\frac{2 \beta}{n} \leq 1$, then for any $\eta>0, C_{G}$ is an $(\varepsilon, \eta)$-differentially private algorithm, where

$$
\varepsilon=\sqrt{2 T \log \left(\frac{1}{\eta}\right)} \cdot \frac{\beta}{n}+4(e-1) \frac{T \beta^{2}}{n^{2}} .
$$

Proof: For any codebook $C$ and $\boldsymbol{z}, \boldsymbol{z}^{\prime} \in\left(\mathbb{R}^{d}\right)^{n}$ such that $\mathrm{d}_{\mathrm{H}}\left(\boldsymbol{z}, \boldsymbol{z}^{\prime}\right)=1$ it holds that

$$
\left|\mathbf{p}_{\boldsymbol{z}}(C \mid j)-\mathbf{p}_{\boldsymbol{z}^{\prime}}(C \mid j)\right| \leq \frac{1}{n}
$$

and so also

$$
\left|\mathbf{p}_{\boldsymbol{z}}(C)-\mathbf{p}_{\boldsymbol{z}^{\prime}}(C)\right|=\left|\frac{1}{m} \sum_{j \in[m]} \mathbf{p}_{\boldsymbol{z}}(C \mid j)-\mathbf{p}_{\boldsymbol{z}^{\prime}}(C \mid j)\right| \leq \frac{1}{n} .
$$

This bound along with the definition of the Gibbs algorithm (29) imply that (as in the proof of Lemma 18)

$$
\left|\log \frac{\mathbb{P}\left[C_{t+1} \mid \boldsymbol{z}, C_{t}\right]}{\mathbb{P}\left[C_{t+1} \mid \boldsymbol{z}^{\prime}, C_{t}\right]}\right| \leq \frac{2 \beta}{n}
$$

Thus, the algorithm $P_{C_{t+1} \mid z, C_{t}}$ is $\left(\frac{2 \beta}{n}, 0\right)$-differentially private for all $t \in[T]$. The algorithm $C_{\mathrm{G}}$ is an adaptive composition [25, Sec. 2.1] of the algorithms $\left(C_{1}, C_{2}, \ldots, C_{T}\right)$, for which, in general, $C_{t}$ depends on the data $z$ and the previous outputs $\left(C_{1}, \ldots, C_{t-1}\right)$ (in this case it only depends on $C_{t-1}$ in a nontrivial way). By the simple composition theorem (e.g., [25, Thm. 3]), $C_{\mathrm{G}}$ is $\left(\frac{2 \beta T}{n}, 0\right)$-differentially private. Furthermore, under the assumption $\frac{2 \beta}{n}<1$, the advanced composition theorem [25. Thm. 4] implies that for any chosen $\eta>0, C_{\mathrm{G}}$ is $(\varepsilon, \eta)$-differentially private with

$$
\varepsilon=\sqrt{2 T \log \left(\frac{1}{\eta^{\prime}}\right)} \cdot \frac{\beta}{n}+T \frac{2 \beta}{n}\left(e^{2 \beta / n}-1\right) \leq \sqrt{2 T \log \left(\frac{1}{\eta^{\prime}}\right)} \cdot \frac{\beta}{n}+4(e-1) \frac{T \beta^{2}}{n^{2}}
$$

where the inequality follows from $e^{x}-1 \leq(e-1) x$ for $x \in[0,1]$.

We may now turn to the proof of Theorem 5 .

Proof of Theorem 5. If $P_{C \mid \boldsymbol{Z}}$ is $(\varepsilon, \eta)$-differentially private then for any given $\boldsymbol{z}, \boldsymbol{z}^{\prime} \in\left(\mathbb{R}^{d}\right)^{n}$ such that $\mathrm{d}_{\mathrm{H}}\left(\boldsymbol{z}, \boldsymbol{z}^{\prime}\right)=1$ it can be verified by standard approximations (or, e.g., [26, Lemma 6]) that for any $\tilde{z} \in \mathbb{R}^{d}$

$$
\left|\mathbf{q} \tilde{z}(C(\boldsymbol{z}))-\mathbf{q} \tilde{z}\left(C\left(\boldsymbol{z}^{\prime}\right)\right)\right| \leq e^{\varepsilon}-1+\eta
$$

Thus, the algorithm $P_{C \mid Z}$ is $\gamma$-uniformly stable w.r.t. the loss function $\mathbf{q}_{\tilde{z}}(\cdot) \in[0,1]$, with $\gamma=e^{\varepsilon}-1+\eta$. Then, [34, Thm 1.1] implies that there exists an absolute constant $c>0$ such that

$$
\mathbb{P}\left[\mathbf{q}_{\mu}\left(C_{\boldsymbol{Z}, T}\right)-\mathbf{q}_{\boldsymbol{Z}}\left(C_{\boldsymbol{Z}, T}\right)>c\left(\gamma+\frac{1}{\sqrt{n}}\right) \log (n) \cdot \log \frac{n}{\delta}\right] \leq \delta
$$


where $\mathbf{q}_{\mu}\left(C_{\boldsymbol{Z}, T}\right):=\mathbb{E}\left[\mathbf{q}_{\tilde{Z}}(C(\boldsymbol{Z}))\right]$ where $\tilde{Z} \sim \mu$ and independent of $\boldsymbol{Z}$, and where $\mathbf{q}_{\boldsymbol{Z}}\left(C_{\boldsymbol{Z}, T}\right):=\frac{1}{n} \sum_{i=1}^{n} \mathbf{q}_{\boldsymbol{Z}}(C(\boldsymbol{Z}))$. By Lemma 19, for the Gibbs algorithm $C_{\mathrm{G}}$, a valid uniform stability parameter is

$$
\gamma=\exp \left[\sqrt{2 T \log \left(\frac{1}{\eta}\right)} \cdot \frac{\beta}{n}+4(e-1) \frac{T \beta^{2}}{n^{2}}\right]-1+\eta
$$

for any $\eta>0$. Under the assumption $\frac{T \beta}{n} \rightarrow 0$ as $n \rightarrow \infty$, there exists $n_{0}$ such that for all $n>n_{0}$

$$
\gamma \leq(e-1) \sqrt{2 T \log \left(\frac{1}{\eta}\right)} \cdot \frac{\beta}{n}+4(e-1)^{2} \frac{T \beta^{2}}{n^{2}}+\eta .
$$

Choosing $\eta=\frac{\sqrt{T} \beta}{n}$, there exists $n_{1}$ such that for all $n>n_{1}$

$$
\gamma \leq \sqrt{18 \log \left(\frac{n}{\beta \sqrt{T}}\right)} \cdot \frac{\sqrt{T} \beta}{n} .
$$

Inserting into 204 completes the proof.

E. The Proofs of Theorems 6 and 7

To prove Theorem 6, we need a few supporting lemmas. Note that $\|Z\|$ has a density $\mu_{\|Z\|}$, and let $\bar{\alpha}_{\mu_{\|Z\|}}:=$ $\inf \left\{\alpha: \mu_{\|Z\|}([0, \alpha])=1\right\} \in(0, \infty]$. For any given $\alpha<\bar{\alpha}_{\mu_{\|Z\|}}$, let $U$ be the following "compressed norm" version of the noise to maximal norm $\alpha$, concretely

$$
U_{\alpha}= \begin{cases}Z, & \|Z\| \leq \alpha \\ \frac{Z}{\|Z\|} \cdot V & \text { otherwise }\end{cases}
$$

where $Z \Perp V \sim$ Uniform $[0, \alpha]$. The Wasserstein distance from the density $\mu_{U_{\alpha}}$ to $\mu_{Z}$ satisfies the following bound:

Lemma 20. Assume that $\|Z\|$ is $\sqrt{d} \sigma_{Z}$-sub-Gaussian. Then, for any $\alpha \in\left(0, \bar{\alpha}_{\mu_{\|Z\|}}\right)$

$$
W_{2}\left(\mu_{Z}, \mu_{U_{\alpha}}\right) \leq \sqrt{2}\left(\alpha+\sqrt{d} \sigma_{Z}\right) \cdot e^{-\alpha^{2} / 2 d \sigma_{Z}^{2}}
$$

Proof: Let $\left(Z^{*}, U^{*}\right)$ be the coupling defined by 208$)$. Then,

$$
\begin{aligned}
W_{2}^{2}\left(\mu_{Z}, \mu_{U}\right) & \leq \mathbb{E}\left\|U^{*}-Z^{*}\right\|^{2} \\
& \leq \mathbb{E}\left[\|Z\|^{2} \cdot \mathbb{1}\{\|Z\|>\alpha\}\right] \\
& \stackrel{(a)}{=} \int_{0}^{\infty} \mathbb{P}\left[\|Z\|^{2} \cdot \mathbb{1}\{\|Z\|>\alpha\}>t\right] \mathrm{d} t \\
& =\int_{0}^{\infty} \mathbb{P}[\|Z\|>\alpha \vee \sqrt{t}] \mathrm{d} t \\
& =\int_{0}^{\alpha^{2}} \mathbb{P}[\|Z\|>\alpha] \mathrm{d} t+\int_{\alpha^{2}}^{\infty} \mathbb{P}[\|Z\|>\sqrt{t}] \mathrm{d} t \\
& \stackrel{(b)}{\leq} \alpha^{2} e^{-\alpha^{2} / d \sigma_{Z}^{2}}+2 \int_{\alpha^{2}}^{\infty} e^{-t / d \sigma_{Z}^{2}} \mathrm{~d} t
\end{aligned}
$$




$$
=\alpha^{2} e^{-\alpha^{2} / d \sigma_{Z}^{2}}+2 d \sigma_{Z}^{2} e^{-\alpha^{2} / d \sigma_{Z}^{2}},
$$

where $(a)$ follows from the integral identity (e.g., [118, Lemma 1.2.1]), and (b) follows from the assumption that $\|Z\|$ is $\left(\sqrt{d} \sigma_{Z}\right)$-sub-Gaussian.

We next bound the Wasserstein distance $W_{2}\left(\mu_{Z}, \mu_{\hat{Z}_{n}}\right)$ where $\mu_{\hat{Z}_{n}}=\sum_{i=1}^{n} \frac{1}{n} \delta_{Z_{i}}$ is the empirical measure of the noise samples $Z$. For the case in which $\|Z\| \leq \alpha$ almost surely, [66, proof of Thm. 3.1] obtained a high probability bound on $W_{1}\left(\mu_{Z}, \mu_{\hat{Z}_{n}}\right)$ utilizing an upper bound on its expected value from [23] and McDiarmid's inequality. We use next similar reasoning and a truncation argument for the more general case which only assumes sub-Gaussian $\|Z\|$, and also bound the second-order distance rather than the first-order.

\section{Lemma 21. Let}

$$
f_{d}(n):= \begin{cases}n^{-1 / 4}, & d<4 \\ n^{-1 / 4} \cdot \log n, & d=4 \\ n^{-1 / d}, & d>4\end{cases}
$$

Assume that $\|Z\|$ is $\sqrt{d} \sigma_{Z}$-sub-Gaussian. For any given $\delta \in(0,1)$, there exists an constant $c_{d}>0$ which only depends on $d$ and $n_{0}\left(\sigma_{Z}, d\right)$ such that for any $n \geq n_{0}$

$$
W_{2}\left(\mu_{Z}, \mu_{\hat{Z}_{n}}\right) \leq c_{d}^{\prime} \sigma_{Z} \log \frac{n}{\delta} \cdot f_{d}(n) \leq c_{d}^{\prime} \sigma_{Z} \log ^{2}\left(\frac{n}{\delta}\right) \cdot n^{-1 /(d \vee 4)}
$$

with probability larger than $1-\delta$.

Proof: For a given $\alpha>0$, let $U_{\alpha}$ be as in 208) and let $\mu_{U}$ denote its probability measure, where for brevity, we omit henceforth in the proof the subscript $\alpha$. Also let $\boldsymbol{U}=\left(U_{1}, \ldots, U_{n}\right) \stackrel{\text { i.i.d. }}{\sim} \mu_{U}$ and let $\mu_{\hat{U}_{n}}:=\frac{1}{n} \sum_{i=1}^{n} \delta_{U_{i}}$ be its empirical measure.

We first derive a high probability bound on $W_{2}\left(\mu_{U}, \mu_{\hat{U}_{n}}\right)$. To this end, we utilize the fact that $\|U\| \leq \alpha$ almost surely, and use a bound on the average Wasserstein distance [37] as follows:

$$
\begin{array}{rlr}
\mathbb{E}\left[W_{2}\left(\mu_{U}, \mu_{\hat{U}_{n}}\right)\right] & \stackrel{(a)}{\leq} \sqrt{\mathbb{E}\left[W_{2}^{2}\left(\mu_{U}, \mu_{\hat{U}_{n}}\right)\right]} \\
& =\stackrel{(b)}{\leq} c_{d} \cdot \alpha \begin{cases}n^{-1 / 4}, & d<4 \\
n^{-1 / 4} \cdot \log n, & d=4 \\
n^{-1 / d}, & d>4\end{cases} \\
& =c_{d} \alpha \cdot f_{d}(n),
\end{array}
$$

where $(a)$ follows from Jensen's inequality, and $(b)$ follows directly from [37, Thm. 1] by taking, in the notation there, $p=2, q \rightarrow \infty$, and $c_{d}$ is a constant which only depends on $d$.

Now, $\boldsymbol{U} \mapsto W_{2}\left(\mu_{U}, \mu_{\hat{U}_{n}}\right)$ satisfies a bounded difference inequality with parameter $\frac{2 \sqrt{d} \alpha}{n}$, just as was noticed in 
[66, proof of Thm. 3.1] for the first-order Wasserstein distance. Hence, by McDiarmid's inequality [12, Thm. 6.2]

$$
\mathbb{P}\left[W_{2}\left(\mu_{U}, \mu_{\hat{U}_{n}}\right)-\mathbb{E}\left[W_{2}\left(\mu_{U}, \mu_{\hat{U}_{n}}\right)\right]>\alpha \sqrt{\frac{2 d}{n} \log \frac{2}{\delta}}\right] \leq \frac{\delta}{2},
$$

and so 221 and 222 imply that with probability larger than $1-\delta / 2$

$$
W_{2}\left(\mu_{U}, \mu_{\hat{U}_{n}}\right) \leq c_{d} \alpha \cdot f_{d}(n)+\alpha \sqrt{\frac{2 d}{n} \log \frac{2}{\delta}} .
$$

We now choose $\alpha>0$ to be large enough so that $W_{2}\left(\mu_{\hat{U}_{n}}, \mu_{\hat{Z}_{n}}\right)=0$ with probability larger than $1-\delta / 2$. To this end, consider the coupling $\left(Z_{i}, U_{i}\right)$ defined by 208 for all $i \in[n]$. Then,

$$
W_{2}\left(\mu_{\hat{U}_{n}}, \mu_{\hat{Z}_{n}}\right) \leq \sqrt{\frac{1}{n} \sum_{i=1}^{n}\left\|Z_{i}-U_{i}\right\|^{2}}
$$

So, if $Z_{i}=U_{i}$ for all $i \in[n]$ then this upper bound is zero. Since $\mathbb{P}[\|Z\|>\sqrt{d} \alpha] \leq \exp \left[-\frac{c_{0} \alpha^{2}}{\sigma_{Z}^{2}}\right]$ for some absolute constant $c_{0}>0$, it holds for $\alpha=\frac{\sigma_{Z}}{\sqrt{c_{0}}} \sqrt{d \log \frac{2 n}{\delta}}$ that

$$
\mathbb{P}\left[\bigcap_{i=1}^{n}\left\{Z_{i}=U_{i}\right\}\right]=\mathbb{P}\left[\bigcap_{i=1}^{n}\left\{\left\|Z_{i}\right\| \leq \alpha\right\}\right] \geq\left[1-e^{-\frac{c_{0} \alpha^{2}}{d \sigma_{Z}^{2}}}\right]^{n} \geq 1-\frac{\delta}{2}
$$

(the last inequality can be verified using the basic inequality $x^{r} \leq 1+r(x-1)$ for $x=1-\delta / 2>0$ and $\left.r=\frac{1}{n} \in[0,1]\right)$.

Consequently, combining the triangle inequality and Lemma 20, 223, and 225, that there exists $n_{0}\left(\sigma_{Z}, d\right)$ such that for all $n \geq n_{0}$

$$
\begin{aligned}
W_{2}\left(\mu_{Z}, \mu_{\hat{Z}_{n}}\right) & \leq W_{2}\left(\mu_{Z}, \mu_{U_{\alpha}}\right)+W_{2}\left(\mu_{U_{\alpha}}, \mu_{\hat{U}_{\alpha, n}}\right)+W_{2}\left(\mu_{\hat{U}_{n}}, \mu_{\hat{Z}_{n}}\right) \\
& =\frac{\sigma_{Z} \sqrt{d \delta}}{\sqrt{2 n}}+c_{d} \frac{\sigma_{Z}}{\sqrt{c_{0}}} \sqrt{d \log \frac{2 n}{\delta}} \cdot f_{d}(n)+\frac{\sigma_{Z}}{\sqrt{c_{0}}} \sqrt{\frac{2 d}{n} \cdot \log \left(\frac{2 n}{\delta}\right) \cdot \log \left(\frac{2}{\delta}\right)} \\
& \leq c_{d}^{\prime} \sigma_{Z} \cdot \log \left(\frac{n}{\delta}\right) \cdot f_{d}(n)
\end{aligned}
$$

with probability larger than $1-\delta$, where $c_{d}^{\prime}>0$ is a constant which only depends on $d$.

We next bound the change in the entropy of the output $Y=X+Z$ when the noise is replaced by its truncated version $U_{\alpha}$. To this end we remind the reader the following simple bounds on the differential entropy of a mixture distribution (see, e.g., [86]).

Lemma 22. Let $\left\{\nu_{j}\right\}_{j \in[m]}$ be a set of density functions such that $\left|h\left(\nu_{j}\right)\right|<\infty$ for all $j \in[m]$, and consider the mixture distribution $f=\sum_{j=1}^{m} a_{j} \nu_{j}$ where $\boldsymbol{a} \in \mathbb{A}^{m-1}$ is a probability vector. Then,

$$
\sum_{j=1}^{m} a_{j} h\left(\nu_{j}\right) \leq h(f) \leq \sum_{j=1}^{m} a_{j} h\left(\nu_{j}\right)+H(\boldsymbol{a})
$$

where $H(\boldsymbol{a}):=-\sum_{j=1}^{m} a_{j} \log a_{j}$ is the (discrete) entropy of $\boldsymbol{a}$. 
Proof: Let $J \in[m]$ be a random variable such that $\mathbb{P}[J=j]=a_{j}$, and $X_{j} \sim \nu_{j}$, where $\left(J, X_{1}, \ldots, X_{m}\right)$ are pairwise independent. Then the left inequality follows from $h(f)=h\left(X_{J}\right) \geq h\left(X_{J} \mid J\right)$, and the right inequality from $H(J) \geq I\left(X_{J} ; J\right)=h\left(X_{J}\right)-h\left(X_{J} \mid J\right)$.

Lemma 23. Suppose that $\mathbb{E}\left\|X^{2}\right\| \leq d \sigma_{X}^{2},|h(X)| \leq \eta_{X}$, and $\|Z\|$ is $\sqrt{d} \sigma_{Z}$-sub-Gaussian. Then, for any $\alpha \in$ $\left(0, \bar{\alpha}_{\mu_{\|Z\|}}\right)$

$$
\left|h\left(X+U_{\alpha}\right)-h(X+Z)\right| \leq\left(2 q \eta_{X}\right) \vee\left(q d \log \left[4 \pi e\left(\left(\sigma_{X}^{2}+\left(d^{-1} \alpha^{2}\right) \vee\left(c q^{-1} \sigma_{Z}^{2}\right)\right)\right]\right)+\mathrm{H}_{\mathrm{bin}}(q)\right.
$$

where $q=\mathbb{P}[\|Z\|>\alpha], c>0$ is an absolute constant, and $\mathrm{H}_{\mathrm{bin}}(t):=-t \log t-(1-t) \log (1-t)$ for $t \in(0,1)$ is the binary entropy function.

Proof: Consider the set $S=\left\{Z \in \mathbb{R}^{d}:\|Z\|<\alpha\right\}$. Let $\mu_{Z \mid S}$ be the measure $\mu_{Z}$ conditioned on the set $S$, i.e., the measure uniquely defined by

$$
\mu_{Z \mid S}(B \cap S)=\frac{\mu_{Z}(B \cap S)}{\mu_{Z}(S)}
$$

for any Borel set of $\mathbb{R}^{d}$. Then, $\mu_{X+Z}=(1-q) \cdot \mu_{Z \mid S} * \mu_{X}+q \cdot \mu_{Z \mid S^{c}} * \mu_{X}$ where $q=\mu_{Z}\left(S^{c}\right)$. Similarly, $\mu_{X+U}=(1-q) \cdot \mu_{Z \mid S} * \mu_{X}+q \cdot \nu * \mu_{X}$ where $\nu$ is defined via the truncation operation (208). Thus, both $\mu_{X+Z}$ and $\mu_{X+U}$ are mixtures of two components, and so Lemma 22 implies that

$$
\begin{aligned}
\left|h\left(X+U_{\alpha}\right)-h(X+Z)\right| & \leq q \cdot\left|h\left(\nu * \mu_{X}\right)-h\left(\nu * \mu_{X}\right)\right|+\mathrm{H}_{\mathrm{bin}}(q) \\
& \leq q \cdot\left|h\left(\nu * \mu_{X}\right)\right|+q \cdot\left|h\left(\mu_{Z \mid S^{c}} * \mu_{X}\right)\right|+\mathrm{H}_{\mathrm{bin}}(q) .
\end{aligned}
$$

We bound each of the entropies in the last display in a similar fashion. Let $V \sim \nu$ such that $X \Perp V$. Then, since Gaussian random vector whose covariance matrix is proportional to the identity matrix maximizes differential entropy under variance constraint (as easily follows, e.g., from Hadamard's inequality [17, Thm. 17.9.2])

$$
\begin{aligned}
h\left(\nu * \mu_{X}\right) & =h(X+V) \\
& \leq \frac{d}{2} \log \left(2 \pi e \mathbb{E}\left[\frac{1}{d}\|X+V\|^{2}\right]\right) \\
& \leq \frac{d}{2} \log \left(4 \pi e \mathbb{E}\left[\frac{1}{d}\|X\|^{2}+\frac{1}{d}\|V\|^{2}\right]\right) \\
& \leq \frac{d}{2} \log \left(4 \pi e\left(\sigma_{X}^{2}+d^{-1} \alpha^{2}\right]\right) .
\end{aligned}
$$

Since also $h(X+V) \geq h(X+V \mid V)=h(X \mid V)=h(X) \geq-\eta_{X}$ we obtain

$$
\left|h\left(\nu * \mu_{X}\right)\right| \leq \eta_{X} \vee \frac{d}{2} \log \left(4 \pi e\left(\sigma_{X}^{2}+d^{-1} \alpha^{2}\right]\right) .
$$

Note that conditioned on $\|Z\|>\alpha$, the density of $Z$ is $\mu_{Z \mid S^{c}}$. Now, using the assumption that $\|Z\|$ is $\left(\sqrt{d} \sigma_{Z}\right)$-sub- 
Gaussian, there exists an absolute constant $c>0$ such that

$$
\mathbb{E}\left[\|Z\|^{2} \mid\|Z\|>\alpha\right]=\frac{\mathbb{E}\left[\|Z\|^{2} \cdot \mathbb{1}\{\|Z\|>\alpha\}\right]}{q} \leq \frac{\mathbb{E}\left[\|Z\|^{2}\right]}{q} \leq \frac{c d \sigma_{Z}^{2}}{q} .
$$

Thus, we obtain, similarly to the bound on $\left|h\left(\nu * \mu_{X}\right)\right|$ that

$$
\left|h\left(\nu * \mu_{Z \mid S^{c}}\right)\right| \leq \eta_{X} \vee \frac{d}{2} \log \left(4 \pi e\left(\sigma_{X}^{2}+c q^{-1} \sigma_{Z}^{2}\right)\right)
$$

We may now prove Theorem 6 .

Proof of Theorem 6. Let $Z_{1}, \ldots, Z_{n}$ be $n$ independent samples from $\mu_{Z}$. By the assumption that $\|Z\|$ is $\sqrt{d} \sigma_{Z}$-sub-Gaussian, there exists a minimal absolute constant $c>0$ such that if $\alpha_{n}=c_{\alpha} \sigma_{Z} \sqrt{2 d \log \frac{n^{2}}{2}}$ then $q \equiv q_{n}:=\mathbb{P}\left[\|Z\|>\alpha_{n}\right] \leq \frac{1}{2 n^{2}}$. If $c_{\alpha}<1 / \sqrt{2}$ we will increase it in the definition of $\alpha_{n}$ to some arbitrary $c_{\alpha}>\frac{1}{\sqrt{2}}$. Then, by the union bound, $\max _{1 \leq i \leq n}\left\|Z_{i}\right\| \leq \alpha_{n}$ with probability larger than $1-\frac{1}{2 n}$. We will assume from this point onward that this event holds.

For any $X \sim \mu_{X} \in \mathcal{P}^{*}$ it holds that

$$
\left|h(X+Z)-h\left(X+\hat{Z}_{n}\right)\right| \leq\left|h(X+Z)-h\left(X+U_{\alpha_{n}}\right)\right|+\left|h\left(X+U_{\alpha_{n}}\right)-h\left(X+\hat{Z}_{n}\right)\right|=: G_{1}+G_{2} .
$$

Analysis of $G_{1}$ : Since $q_{n} \leq \frac{1}{2 n^{2}} \leq \frac{1}{2}$ and as $-\log (1-t) \leq t$ for $t \in(0,1 / 2)$, we may bound

$$
\mathrm{H}_{\text {bin }}\left(q_{n}\right) \leq \mathrm{H}_{\text {bin }}\left(\frac{1}{2 n^{2}}\right)=\frac{\log \left(2 n^{2}\right)}{2 n^{2}}-\left(1-\frac{1}{2 n^{2}}\right) \log \left(1-\frac{1}{2 n^{2}}\right) \leq \frac{2 \log n+\log (2 e)}{2 n^{2}}
$$

Substituting this bound in Lemma 23, we deduce that there exists $n_{0} \in \mathbb{N}$ and $C_{0}>0$, both which depend on $\left(d, \sigma_{X}^{2}, \eta_{X}, \sigma_{Z}^{2}\right)$, such that

$$
G_{1} \leq C_{0} \frac{\log n}{n^{2}}
$$

Indeed, to evaluate the asymptotic order of the term inside the parenthesis in 230, we note that $q \mapsto q \log q^{-1}$ is monotonic increasing on $\left[0, \frac{1}{4}\right]$ and so $\max _{0 \leq q \leq n^{-2}} q \log q^{-1}=2 \frac{\log n}{n^{2}}$ for $n \geq 2$.

Analysis of $G_{2}$ : By definition $\left\|U_{\alpha_{n}}\right\| \leq \alpha_{n}$, and under the high probability assumption, $\left\|\hat{Z}_{n}\right\| \leq \alpha_{n}$ holds too. It was shown in [92, Prop. 3] that if $V=B+W$ where $B \Perp W,\|B\|<\sigma^{2} d$ with probability 1 , and $W$ is $\left(\psi_{1}, \psi_{2}\right)$-regular, then $\mu_{V}$ is $\left(\psi_{1}, \psi_{2}+\psi_{1} \sigma \sqrt{d}\right)$-regular. Since $\mu_{X}$ is $\left(\psi_{1}, \psi_{2}\right)$-regular by assumption, it holds that both $X+U_{\alpha_{n}}$ and $X+\hat{Z}_{n}$ are $\left(\psi_{1}, \tilde{\psi}_{2}\right)$-regular with $\tilde{\psi}_{2}:=\psi_{2}+\psi_{1} c_{\alpha} \sigma_{Z} \sqrt{4 d \log n}$. Then, by [92, Prop. 1], it holds with probability larger than $1-\frac{1}{n}$ that

$$
G_{2} \leq\left(\psi_{1} \sqrt{\mathbb{E}\left[\left\|X+\hat{Z}_{n}\right\|^{2}\right]}+\psi_{1} \sqrt{\mathbb{E}\left[\left\|X+U_{\alpha_{n}}\right\|^{2}\right]}+\tilde{\psi}_{2}\right) \cdot W_{2}\left(\mu_{X+\hat{Z}_{n}}, \mu_{X+U_{\alpha_{n}}}\right) .
$$

To bounds the r.h.s. of 244, we note that with probability larger than $1-\frac{1}{2 n}$ there exists a constant $C_{1}\left(d, \sigma_{Z}\right)$ 
such that

$$
\begin{aligned}
W_{2}\left(\mu_{X+\hat{Z}_{n}}, \mu_{X+U_{\alpha_{n}}}\right) & \stackrel{(a)}{\leq} W_{2}\left(\mu_{\hat{Z}_{n}}, \mu_{U_{\alpha_{n}}}\right) \\
& \stackrel{(b)}{\leq} W_{2}\left(\mu_{\hat{Z}_{n}}, \mu_{Z}\right)+W_{2}\left(\mu_{Z}, \mu_{U_{\alpha_{n}}}\right) \\
& \stackrel{(c)}{\leq} c_{d}^{\prime} \sigma_{Z} \log ^{2}\left(4 n^{2}\right) \cdot n^{-1 /(d \vee 4)}+\sqrt{2}\left(\alpha_{n}+\sqrt{d} \sigma_{Z}\right) e^{-\alpha_{n}^{2} / 2 d \sigma_{Z}^{2}} \\
& \stackrel{(d)}{\leq} C_{1}\left(d, \sigma_{Z}\right) \frac{\log ^{2} n}{n^{1 /(d \vee 4)}},
\end{aligned}
$$

where $(a)$ follows since Wasserstein distance is non-increasing under a convolution operation $\sqrt{17}(b)$ follows from the triangle inequality, $(c)$ follows from Lemmas 20 and 21, and $(d)$ utilizes the assumptions $c_{\alpha}>\frac{1}{\sqrt{2}}$ and $\sigma_{Z}=\Omega\left(n^{-(d-2) /(4 d)}\right)$ to show that $\sqrt{2}\left(\alpha_{n}+\sqrt{d} \sigma_{Z}\right) e^{-\alpha_{n}^{2} / 2 d \sigma_{Z}^{2}}=o\left(n^{-1 / d}\right)$.

Substituting the bound 248 , as well as $\mathbb{E}\left[\left\|X+\hat{Z}_{n}\right\|^{2}\right] \leq 2 d \sigma_{X}^{2}+2 \alpha_{n}^{2}$ and $\mathbb{E}\left[\left\|X+U_{\alpha_{n}}\right\|^{2}\right] \leq 2 d \sigma_{X}^{2}+2 \alpha_{n}^{2}$, in (244), and then using (243) and (248) back in 241) implies that there exists a constant $C_{2}>0$ which depends on $\left(d, \sigma_{X}, \eta_{X}, \psi_{1}, \psi_{2}, \sigma_{Z}\right)$ such that

$$
\sup _{X: \mu_{X} \in \mathcal{P}^{*}}\left|h(X+Z)-h\left(X+\hat{Z}_{n}\right)\right| \leq C_{2} \frac{\log ^{2} n}{n^{1 /(d \vee 4)}}
$$

with probability larger than $1-\frac{1}{n}$, and completes the proof.

We now turn to prove Theorem 7. The next lemma bounds the difference in the differential entropy of two mixtures which have the same component densities but perhaps different mixing weights. The bound is given in terms of the total variation and chi-square distance between the weights of the two densities, and also depends on the maximum second-order Rényi entropy of the component densities.

Lemma 24. Let $\left\{\nu_{j}\right\}_{j \in[m]}$ be a set of density functions such that $\left|h_{2}\left(\nu_{j}\right)\right| \leq A_{\nu, 2}$ for all $j \in[m]$ (where $h_{2}(\cdot)$ is the second-order differential Rényi entropy). Consider the mixture distributions $f=\sum_{j=1}^{m} a_{j} \nu_{j}$ and $g=\sum_{j=1}^{m} b_{j} \nu_{j}$ where $\boldsymbol{a}, \boldsymbol{b} \in \mathbb{A}^{m-1}$ are probability vectors. If $\min _{j \in[m]} b_{j} \geq \epsilon$ then

$$
|h(f)-h(g)| \leq \mathrm{d}_{\mathrm{H}}(\boldsymbol{a}, \boldsymbol{b}) \cdot \mathrm{d}_{\chi^{2}}(\boldsymbol{a}, \boldsymbol{b})+2\left(\log \frac{1}{\epsilon}+A_{\nu, 2}\right) \cdot \mathrm{d}_{\mathrm{TV}}(\boldsymbol{a}, \boldsymbol{b}) .
$$

Proof: By the triangle inequality and the fact the chi-square divergence dominates the KL divergence (e.g. [112, Lemma 2.7])

$$
\begin{aligned}
|h(f)-h(g)| & \leq\left|-\int f \log f+\int f \log g\right|+\left|-\int f \log g+\int g \log g\right| \\
& =\mathrm{d}_{\mathrm{KL}}(f, g)+\left|\int(g-f) \log g\right| \\
& \leq \mathrm{d}_{\chi^{2}}(f, g)+\left|\int(g-f) \log g\right| .
\end{aligned}
$$

\footnotetext{
${ }^{17}$ Namely, if $X \Perp Z_{1}$ and $X \Perp Z_{2}$ and let $U=X+Z_{1}$ and $V=X+Z_{2}$. Then, $W_{p}\left(\mu_{U}, \mu_{V}\right) \leq W_{p}\left(\mu_{Z_{2}}, \mu_{Z_{1}}\right)$.
} 
We obtain the desired bound by bounding each of the terms in the last equation separately. For the first term, using Cauchy-Schwarz inequality,

$$
\begin{aligned}
\mathrm{d}_{\chi^{2}}(f, g) & =\int \frac{\left(\sum_{j: a_{j} \neq b_{j}}\left(a_{j}-b_{j}\right) \nu_{j}\right)^{2}}{\sum b_{j} \nu_{j}} \\
& \leq \int \frac{\left(\sum_{j: a_{j} \neq b_{j}} \frac{\left(a_{j}-b_{j}\right)}{\sqrt{b_{j}}} \sqrt{b_{j}} \nu_{j}\right)^{2}}{\sum_{j: a_{j} \neq b_{j}} b_{j} \nu_{j}} \\
& \leq \int \sum_{j: a_{j} \neq b_{j}} \frac{\left(a_{j}-b_{j}\right)^{2}}{b_{j}} \cdot \frac{\sum_{j: a_{j} \neq b_{j}} b_{j} \nu_{j}^{2}}{\sum_{j: a_{j} \neq b_{j}} b_{j} \nu_{j}} \\
& =\mathrm{d}_{\chi^{2}}(\boldsymbol{a}, \boldsymbol{b}) \int \cdot \frac{\sum_{j: a_{j} \neq b_{j}} b_{j} \nu_{j}^{2}}{\sum_{j: a_{j} \neq b_{j}} b_{j} \nu_{j}} \\
& =\mathrm{d}_{\chi^{2}}(\boldsymbol{a}, \boldsymbol{b}) \cdot \int \max _{j \in[m]: a_{j} \neq b_{j}} \nu_{j} \\
& \leq \mathrm{d}_{\chi^{2}}(\boldsymbol{a}, \boldsymbol{b}) \cdot \sum_{j \in[m]: a_{j} \neq b_{j}} \int \nu_{j} \\
& =\mathrm{d}_{\chi^{2}}(\boldsymbol{a}, \boldsymbol{b}) \cdot \mathrm{d}_{\mathrm{H}}(\boldsymbol{a}, \boldsymbol{b}) .
\end{aligned}
$$

For the second term, it holds by Jensen and Cauchy-Schwarz inequalities that

$$
\int \nu_{j} \log \left(\sum_{l} b_{l} \nu_{l}\right) \leq \log \left(\sum_{l} b_{l} \int \nu_{j} \nu_{l}\right) \leq \log \left(\max _{l} \int \nu_{j} \nu_{l}\right) \leq \max _{l} \frac{1}{2} \log \left(\int \nu_{j}^{2} \int \nu_{l}^{2}\right) \leq A_{\nu, 2} .
$$

Since Rényi entropies are decreasing functions of their order ${ }^{18}$ it holds that $h\left(\nu_{j}\right) \geq h_{2}\left(\nu_{j}\right)$ and so

$$
\int \nu_{j} \log \left(\sum_{l} b_{l} \nu_{l}\right) \geq \int \nu_{j} \log \left(b_{j} \nu_{j}\right)=-\log \frac{1}{\epsilon}-h\left(\nu_{j}\right) \geq-\log \frac{1}{\epsilon}-A_{\nu, 2} .
$$

The upper bound then follows from combining the above two bounds and

$$
\left|\int(g-f) \log g\right|=\left|\sum_{j}\left(b_{j}-a_{j}\right) \int \nu_{j} \log \left(\sum_{l} b_{l} \nu_{l}\right)\right| \leq \sum_{j}\left|b_{j}-a_{j}\right| \cdot\left|\int \nu_{j} \log \left(\sum_{l} b_{l} \nu_{l}\right)\right| .
$$

The next lemma provides an upper bound on the second-order Rényi entropy of $\tilde{Z}_{n}$.

Lemma 25. Suppose that $\tilde{Z}_{n} \sim \frac{1}{\theta^{d}} \sum_{i=1}^{n} a_{i} \kappa_{\theta, Z_{i}}:=\mu_{\tilde{Z}_{n}}$, where $\left|h_{2}(\kappa)\right| \leq A_{\kappa, 2}, a_{i} \geq \epsilon>0$ for all $i \in[n]$, and $\theta<1$. Then,

$$
\left|h_{2}\left(\tilde{Z}_{n}\right)\right| \leq A_{\tilde{Z}, 2}:=A_{\kappa, 2}+d \log \frac{1}{\theta}+2 \log \frac{1}{\epsilon} .
$$

${ }^{18}$ For example, [114. Eq. (3) and Thm. 3] state this for densities with finite support and proves this by a related property for the Rényi divergence, but the result holds for general densities and can be proved similarly to the discrete case. 
Proof: By Jensen's inequality

$$
\int \mu_{\tilde{Z}_{n}}^{2}=\int\left(\frac{1}{\theta^{d}} \sum_{i=1}^{n} a_{i} \kappa_{\theta, Z_{i}}\right)^{2} \leq \frac{1}{\theta^{2 d}} \sum_{i=1}^{n} a_{i} \int \kappa_{\theta, Z_{i}}^{2} \leq \frac{1}{\theta^{2 d}} \int \kappa^{2}\left(\frac{z}{\theta}\right) \mathrm{d} z=\frac{1}{\theta^{d}} \int \kappa^{2} \leq \frac{e^{A_{\kappa, 2}}}{\theta^{d}} .
$$

Furthermore,

$$
\int \mu_{\tilde{Z}_{n}}^{2}=\int\left(\frac{1}{\theta^{d}} \sum_{i=1}^{n} a_{i} \kappa_{\theta, Z_{n}}\right)^{2} \geq \frac{\epsilon^{2}}{\theta^{2 d}} \int\left(\max _{i \in[n]} \kappa_{\theta, Z_{i}}\right)^{2} \geq \frac{\epsilon^{2}}{\theta^{2 d}} \max _{i \in[n]} \int\left(\kappa_{\theta, Z_{i}}\right)^{2} \geq \frac{\epsilon^{2} e^{-A_{\kappa .2}}}{\theta^{d}} .
$$

The results then follows by combining both bounds.

The next lemma states that the entropy difference $\left|h\left(X_{\boldsymbol{a}}+Z\right)-h\left(X_{\boldsymbol{a}}+\tilde{Z}_{n}\right)\right|$ concentrates fast around its mean value.

Lemma 26. Let $\epsilon>0$ be given. Let $\Gamma(\boldsymbol{Z}):=\left|h\left(X_{\boldsymbol{a}}+Z\right)-h\left(X_{\boldsymbol{a}}+\tilde{Z}_{n}\right)\right|$ where $X_{\boldsymbol{a}} \sim \sum_{j=1}^{m} a_{j} \delta_{x_{j}}$ and $a_{j} \geq \epsilon>0$ for all $j \in[m]$, and $\tilde{Z}_{n} \sim \frac{1}{n \theta^{d}} \sum_{i=1}^{n} \kappa_{\theta, Z_{i}}$ where $\left|h_{2}(\kappa)\right| \leq A_{\kappa, 2}$. Then, for any $\delta_{1} \in(0,1)$

$$
\Gamma(\boldsymbol{Z}) \leq \mathbb{E} \Gamma(\boldsymbol{Z})+\sqrt{\frac{\left(6+2 \log n+A_{\kappa_{C}}\right)^{2}}{2 n} \log \frac{1}{\delta_{1}}}
$$

with probability larger than $1-\delta_{1}$, where

$$
A_{\kappa_{C}}:=A_{\kappa, 2}+d \log \frac{1}{\theta}+2 \log \frac{1}{\epsilon} .
$$

Proof: Let $\boldsymbol{z}^{(0)}, \boldsymbol{z}^{(1)} \in \mathbb{R}^{d}$ be such that $\mathrm{d}_{\mathrm{H}}\left(\boldsymbol{z}^{(0)}, \boldsymbol{z}^{(1)}\right) \leq 1$, and further let $\tilde{Z}_{n}^{(l)} \sim \frac{1}{n \theta^{d}} \sum_{i=1}^{n} \kappa_{\theta, z_{i}^{(l)}}$ for $l=0,1$ be the corresponding KDEs of $\mu_{Z}$, and assume w.l.o.g. that $z_{i}^{(0)}=z_{i}^{(1)}$ for all $i \in[n-1]$. Let $\bar{z}=$ $\left(z_{1}^{(0)}, z_{2}^{(0)}, \ldots, z_{n}^{(0)}, z_{n}^{(1)}\right) \in \mathbb{R}^{n+1}$ and denote

$$
\begin{aligned}
\boldsymbol{q}^{(0)} & =\left(\frac{1}{n}, \ldots, \frac{1}{n}, \frac{1}{n}, 0\right) \in[0,1]^{n+1} \\
\boldsymbol{q}^{(1)} & =\left(\frac{1}{n}, \ldots \frac{1}{n}, 0, \frac{1}{n}\right) \in[0,1]^{n+1} \\
\boldsymbol{q}^{(1 / 2)} & =\left(\frac{1}{n}, \ldots, \frac{1}{n}, \frac{1}{2 n}, \frac{1}{2 n}\right) \in[0,1]^{n+1}
\end{aligned}
$$

such that $\hat{Z}_{n}^{(l)} \sim \sum_{i=1}^{n+1} q_{i}^{(l)} \delta_{\bar{z}_{i}}$ for $l=0,1$. Further denote the "codebook kernel" $\kappa_{C}:=\frac{1}{\theta^{d}} \sum_{j=1}^{m} a_{j} \kappa_{\theta, x_{j}}$, which, by Lemma 25. satisfies that $\left|h_{2}\left(\kappa_{C}\right)\right| \leq A_{\kappa_{C}}$ for $A_{\kappa_{C}}$ in 268. Then, for $V \stackrel{d}{=} X+\theta U \sim \kappa_{C}$ where $U \sim \kappa$ and $U \Perp X$, it holds that

$$
\begin{aligned}
\left|\Gamma\left(\boldsymbol{z}^{(0)}\right)-\Gamma\left(\boldsymbol{z}^{(1)}\right)\right| & \stackrel{(a)}{\leq}\left|h\left(X+\tilde{Z}_{n}^{(0)}\right)-h\left(X+\tilde{Z}_{n}^{(1)}\right)\right| \\
& =\left|h\left(V+\hat{Z}_{n}^{(0)}\right)-h\left(V+\hat{Z}_{n}^{(1)}\right)\right| \\
& \stackrel{(b)}{\leq}\left|h\left(V+\hat{Z}_{n}^{(0)}\right)-h\left(V+\hat{Z}_{n}^{(1 / 2)}\right)\right|+\left|h\left(V+\hat{Z}_{n}^{(1 / 2)}\right)-h\left(V+\hat{Z}_{n}^{(1)}\right)\right| \\
& \stackrel{(c)}{\leq} \frac{4}{n}+\frac{2}{n}\left(\log (2 n)+A_{\kappa_{C}}\right),
\end{aligned}
$$


where $(a)$ follows from the reverse triangle inequality, $(b)$ follows from the triangle inequality, and $(c)$ follows by bounding the two terms in the same manner using Lemma 24 Specifically, for the first term, we set $\epsilon=\frac{1}{2 n}$ and $\nu_{i}=\kappa_{C, \bar{z}_{i}}$ (which is a shift of $\kappa_{C}$ by $Z_{i}$ ) and $a_{i}=q_{i}$ for $i \in[n+1]$ and note that $\boldsymbol{q}^{(1 / 2)} \gg \boldsymbol{q}^{(0)}$, and $\mathrm{d}_{\mathrm{TV}}\left(\boldsymbol{q}^{(0)}, \boldsymbol{q}^{(1 / 2)}\right)=\frac{1}{2 n}, \mathrm{~d}_{\chi^{2}}\left(\boldsymbol{q}^{(0)}, \boldsymbol{q}^{(1 / 2)}\right)=\frac{1}{n}$, and $\mathrm{d}_{\mathrm{H}}\left(\boldsymbol{q}^{(0)}, \boldsymbol{q}^{(1 / 2)}\right)=2$.

Now, the function $\Gamma(z)$ satisfies $\frac{1}{n}\left(6+2 \log n+A_{\kappa_{C}}\right)$-bounded-difference property, and so the stated result follows from McDiarmid's inequality (bounded differences inequality) [12, Thm. 6.2] which implies that for any $t \geq 0$

$$
\mathbb{P}[\Gamma(\boldsymbol{Z})>\mathbb{E} \Gamma(\boldsymbol{Z})+t] \leq \exp \left[-\frac{2 n t^{2}}{\left(6+2 \log n+A_{\kappa_{C}}\right)^{2}}\right]
$$

We may now prove the theorem.

Proof of Theorem 7. Let $\epsilon>0$ be given such that $m^{2} \epsilon<1$ and consider an $\epsilon$-net for [0,1] given by $\mathcal{F}=\{\epsilon, 2 \epsilon, \ldots, 1\}$ where we assume that $\epsilon^{-1}$ is integer. In addition, consider a net $\mathcal{A}_{\epsilon}^{m-1} \subset \mathbb{A}^{m-1}$ constructed by a quantization of $\boldsymbol{a} \in \mathbb{A}^{m-1}$ to $\boldsymbol{b} \in \mathcal{A}_{\epsilon}^{m-1}$ in the following way. Assume for the sake of notational simplicity that $\boldsymbol{a}$ is ordered such that $a_{1} \leq a_{2} \leq \cdots \leq a_{m}$. The first $m-1$ coordinates of $\boldsymbol{a}$ are rounded upwards so that $b_{j}=\min \left\{b \in \mathcal{F}: b \geq a_{j}\right\}$ for any $j \in[m-1]$, and $b_{m}=1-\sum_{j=1}^{m} \bar{a}_{j} \in \mathcal{F}$. So, by construction $b_{j} \geq \epsilon$ for all $j \in[m-1]$, and since by the ordering assumption $a_{m} \geq \frac{1}{m}$ must hold, the condition $m^{2} \epsilon<1$ and the quantization definition imply that $b_{m}>\epsilon$ also holds. The number of possible probability vectors $\boldsymbol{b}$ obtained in this way is at most $\left|\mathcal{A}_{\epsilon}^{m-1}\right| \leq\left(\frac{1}{\epsilon}\right)^{m}$. Furthermore, if $\boldsymbol{a}$ is mapped to $\boldsymbol{b} \in \mathcal{A}_{\epsilon}^{m-1}$ then

$$
\mathrm{d}_{\mathrm{TV}}(\boldsymbol{a}, \boldsymbol{b})=\frac{1}{2} \sum_{j=1}^{m}\left|a_{j}-b_{j}\right| \leq m \epsilon,
$$

and

$$
\mathrm{d}_{\chi^{2}}(\boldsymbol{a}, \boldsymbol{b})=\sum_{j=1}^{m} \frac{\left(a_{j}-b_{j}\right)^{2}}{b_{j}} \leq \sum_{j=1}^{m-1} \frac{\epsilon^{2}}{\epsilon}+\frac{m^{2} \epsilon^{2}}{\epsilon} \leq 2 m^{2} \epsilon .
$$

Hence, for any $\boldsymbol{a} \in \mathcal{A}$ there exists $\boldsymbol{b} \in \mathcal{A}_{\epsilon}^{m-1}$ such that $\mathrm{d}_{\mathrm{TV}}(\boldsymbol{a}, \boldsymbol{b}) \leq m \epsilon$ and $\mathrm{d}_{\chi^{2}}(\boldsymbol{a}, \boldsymbol{b}) \leq 2 m^{2} \epsilon{ }^{19}$

Let $\boldsymbol{a} \in \mathbb{A}^{m-1}$ be mapped to $\boldsymbol{b} \in \mathcal{A}_{\epsilon}^{m-1}$. Then,

$$
\begin{aligned}
& \left|h\left(X_{\boldsymbol{a}}+Z\right)-h\left(X_{\boldsymbol{a}}+\tilde{Z}_{n}\right)\right| \\
& \leq\left|h\left(X_{\boldsymbol{a}}+Z\right)-h\left(X_{\boldsymbol{b}}+Z\right)\right|+\left|h\left(X_{\boldsymbol{b}}+Z\right)-h\left(X_{\boldsymbol{b}}+\tilde{Z}_{n}\right)\right|+\left|h\left(X_{\boldsymbol{a}}+\tilde{Z}_{n}\right)-h\left(X_{\boldsymbol{b}}+\tilde{Z}_{n}\right)\right| \\
& :=G_{1}+G_{2}+G_{3} .
\end{aligned}
$$

Analysis of $G_{1}$ : By the assumption on the Rényi entropy of $\mu_{Z}$ and Lemma 24 with $\nu_{j}(z)=\mu_{Z}\left(z-x_{j}\right)$ it holds that

$$
G_{1} \leq 2 m^{3} \epsilon+2\left(\log \frac{1}{\epsilon}+A_{Z, 2}\right) m \epsilon
$$

\footnotetext{
${ }^{19}$ In other words, $\mathcal{A}_{\epsilon}^{m-1}$ is an $m \epsilon$-cover of $\mathbb{A}^{m-1}$ in the total variation distance and an $\left(2 m^{2} \epsilon\right)$-"cover" of $\mathbb{A}^{m-1}$ in the chi-square divergence (the latter statement, however, is not rigorous since the chi-square divergence is not symmetric and thus not a metric).
} 
Analysis of $G_{2}$ : Take $\delta_{1}=\frac{\delta}{\left|\mathcal{A}_{\epsilon}^{m-1}\right|}$, and denote $\Gamma_{\boldsymbol{b}}(\boldsymbol{Z}):=\left|h\left(X_{\boldsymbol{b}}+Z\right)-h\left(X_{\boldsymbol{b}}+\tilde{Z}_{n}\right)\right|$. Since $b_{j} \geq \epsilon$ for all $j \in[m]$, Lemma 26 and the union bound assure that

$$
\mathbb{P}\left[\bigcap_{\boldsymbol{b} \in \mathcal{A}_{\epsilon}^{m-1}}\left\{\Gamma_{\boldsymbol{b}}(\boldsymbol{Z})<\mathbb{E} \Gamma_{\boldsymbol{b}}(\boldsymbol{Z})+\sqrt{\frac{\left(6+2 \log n+A_{\kappa_{C}}\right)^{2}}{2 n} \log \frac{1}{\delta_{1}}}\right\}\right] \geq 1-\delta,
$$

where $A_{\kappa_{C}}$ is as in 268. Thus, given that this event holds, for any $\boldsymbol{b} \in \mathcal{A}_{\epsilon}^{m-1}$

$$
G_{2} \leq \mathbb{E}\left[\left|h\left(X_{\boldsymbol{b}}+Z\right)-h\left(X_{\boldsymbol{b}}+\tilde{Z}_{n}\right)\right|\right]+\left(6+2 \log n+A_{\kappa_{C}}\right) \sqrt{\frac{1}{2 n} \log \frac{1}{\delta}+\frac{m}{2 n} \log \frac{2}{\epsilon}}
$$

Analysis of $G_{3}$ : It holds that $\tilde{Z}_{n} \sim \frac{1}{n \theta^{d}} \sum_{i=1}^{n} \kappa_{\theta, Z_{i}}:=\mu_{\tilde{Z}_{n}}$, and so by Lemma 25

$$
\left|h_{2}\left(\mu_{\tilde{Z}_{n}}\right)\right| \leq A_{\kappa}+2 \log n+2 d \log \frac{1}{\theta}=: A_{\tilde{Z}, 2} .
$$

Lemma 24 with $\nu_{j}(z)=\mu_{\tilde{Z}_{n}}\left(z-x_{j}\right)$ implies that

$$
G_{3} \leq 2 m^{3} \epsilon+2\left(\log \frac{1}{\epsilon}+A_{\kappa, 2}+2 \log n+2 d \log \frac{1}{\theta}\right) m \epsilon
$$

From the bounds on $G_{1}, G_{2}$ and $G_{3}$ we deduce that it holds with probability larger than $1-\delta$ that

$$
\begin{aligned}
\sup _{\boldsymbol{a} \in \mathbb{A}^{m-1}}\left|h\left(X_{\boldsymbol{a}}+Z\right)-h\left(X_{\boldsymbol{a}}+\tilde{Z}_{n}\right)\right| & \leq \max _{\boldsymbol{b} \in \mathcal{A}_{\epsilon}^{m-1}} \mathbb{E}\left[\left|h\left(X_{\boldsymbol{b}}+Z\right)-h\left(X_{\boldsymbol{b}}+\tilde{Z}_{n}\right)\right|\right]+\Delta_{0}(\epsilon) \\
& \leq \sup _{\boldsymbol{a} \in \mathbb{A}^{m-1}} \mathbb{E}\left[\left|h\left(X_{\boldsymbol{b}}+Z\right)-h\left(X_{\boldsymbol{b}}+\tilde{Z}_{n}\right)\right|\right]+\Delta_{0}(\epsilon),
\end{aligned}
$$

where

$$
\begin{aligned}
\Delta_{0}(\epsilon)= & 4 m^{3} \epsilon+2\left[2 \log \frac{1}{\epsilon}+2 \log n+2 d \log \frac{1}{\theta}+A_{Z, 2}+A_{\kappa, 2}\right] m \epsilon \\
& +\left(6+2 \log n+A_{\kappa, 2}+d \log \frac{1}{\theta}+2 \log \frac{1}{\epsilon}\right) \sqrt{\frac{1}{2 n} \log \frac{1}{\delta}+\frac{m}{2 n} \log \frac{2}{\epsilon}}
\end{aligned}
$$

Choosing $\epsilon=\frac{1}{\left\lceil n m^{2} \sqrt{m}\right\rceil}$ and simplifying ${ }^{20}$ completes the proof.

\section{APPENDIX B}

\section{EXPERIMENTS DETAILS}

We denote by $Q_{\alpha}:=\left[\begin{array}{cc}\cos (\alpha) & \sin (\alpha) \\ -\sin (\alpha) & \cos (\alpha)\end{array}\right]$ a rotation matrix of angle $\alpha$.

\footnotetext{
${ }^{20}$ The ceiling operation in the choice of $\epsilon$ has a negligible effect on the final result.
} 


\begin{tabular}{|c|c|c|c|}
\hline Notation & Description & Value (Sec. VI-A1) & Value (Sec. VI-A2) \\
\hline$d$ & channel dimension & 2 & 4 \\
\hline$m$ & codebook cardinality & 32 & $(8,16,32,64)$ \\
\hline$r_{s}$ & maximal eigenvector of $S$ & 10 & 10 \\
\hline$\phi_{x}$ & input distribution projection parameter & 2 & 2 \\
\hline$\Gamma$ & "gap-to-capacity" parameter & 10 & 20 \\
\hline$n$ & number of training samples & $2 \cdot 10^{3}$ & $2 \cdot 10^{3}$ \\
\hline$Z$ & noise distribution & $Z \sim N(0, K)$ & $\begin{array}{c}Z \stackrel{d}{=} \Psi\left[\alpha\left(\sum_{l=1}^{k} R_{l} \cdot v_{l}+W\right)\right. \\
W \sim N\left(0, K_{W}\right) \\
R_{l} \sim \text { Uniform }\{ \pm 1\}, \text { i.i.d. } \\
W \Perp\left\{R_{l}\right\}\end{array}$ \\
\hline$\phi_{z}$ & noise distribution projection parameter & N/A & 2 \\
\hline$K$ or $K_{W}$ & Gaussian noise covariance matrix & $\begin{array}{c}K=10^{-1} \cdot Q_{\alpha} \Lambda Q_{\alpha}^{T}, \\
\Lambda:=\left[\begin{array}{ll}1 & 0 \\
0 & 3\end{array}\right], \alpha=30^{\circ}\end{array}$ & $\begin{array}{c}K_{W}=A A^{T} \\
A_{i_{1} i_{2}} \sim N(0,1) \text { i.i.d. }\end{array}$ \\
\hline$\left\{v_{l}\right\}_{l=1}^{s}$ & interference vectors & N/A & $v_{l} \sim N\left(0, I_{d}\right)$ i.i.d., $s=5$ \\
\hline$r_{x}$ & maximal power constraint & \multicolumn{2}{|c|}{$r_{x}=\Gamma \cdot r_{\min }$} \\
\hline$C^{(0)}=\left\{X_{j}^{(0)}\right\}_{j=1}^{m}$ & initial codebook generation & \multicolumn{2}{|c|}{$X_{j}^{(0)} \sim N\left(0, \frac{r_{x}^{2}}{\chi^{d}} \cdot I_{d}\right)$ i.i.d. } \\
\hline$\lambda^{(i)} \equiv \lambda$ & SGD step sizes - codeword update & \multicolumn{2}{|r|}{$10^{-1}$} \\
\hline$\eta^{(i)} \equiv \eta$ & SGD step sizes - covariance update & \multicolumn{2}{|r|}{$10^{-1}$} \\
\hline \multirow[t]{3}{*}{$\tilde{n}$} & number of validation samples & $10^{4}$ & $10^{3}$ \\
\hline & total number of runs & N/A & $10^{3}$ \\
\hline & number of runs per distribution & N/A & 10 \\
\hline
\end{tabular}

Table I

SGD ALGORITHM EXPERIMENTS PARAMETERS

\begin{tabular}{|c|c|c|}
\hline Notation & Description & Value (Sec. VI-B) \\
\hline$d$ & channel dimension & 4 \\
\hline$m$ & codebook cardinality & $2 \leq m \leq 64$ \\
\hline$\Gamma$ & "gap-to-capacity" parameter & 10 \\
\hline$n$ & number of training samples & $10^{2}$ \\
\hline$Z$ & noise distribution & $\begin{array}{c}Z \stackrel{d}{=} \sum_{l=1}^{k} R_{l} \cdot v_{l}+W \\
W \sim N\left(0, K_{W}\right), R_{l} \sim \text { Uniform }\{ \pm 1\}\end{array}$ \\
\hline$K_{W}$ & Gaussian noise covariance matrix & $K_{W}=A A^{T}, A_{i_{1} i_{2}} \sim N(0,1)$ i.i.d. \\
\hline$\left\{v_{l}\right\}_{l=1}^{s}$ & interference vectors & $v_{l} \sim N\left(0, I_{d}\right)$ i.i.d., $s=10$ \\
\hline$r_{x}$ & maximal power constraint & $r_{x}=\Gamma \cdot r_{\min }$ \\
\hline$C^{(0)}=\left\{X_{j}^{(0)}\right\}_{j=1}^{m}$ & initial codebook generation & $X_{j}^{(0)} \sim N\left(0, \frac{r_{x}^{2}}{d} \cdot I_{d}\right)$ i.i.d. \\
\hline$m_{0}$ & number of initial codewords & 64 \\
\hline$k$ & number of codewords removed at each stage & 1 \\
\hline$\beta$ & inverse temperature parameter & $(1,10,100) \cdot 10^{2}$ \\
\hline \multirow[t]{2}{*}{$Q(\mathrm{~d} x)$} & reference measure & Lebesgue measure \\
\hline & decoder & minimum distance $S=I_{d}$ \\
\hline \multirow[t]{3}{*}{$\tilde{n}$} & number of validation samples & $10^{4}$ \\
\hline & total number of runs & $2.5 \cdot 10^{3}$ \\
\hline & number of runs per distribution & 10 \\
\hline
\end{tabular}

Table II

GIBBS ALGORITHM EXPERIMENT PARAMETERS 


\section{APPENDIX C}

\section{Memoization Implementation of the Gibbs Algorithm}

The main computational task required by Algorithm 2 is an efficient computation of the average error probability of a codebook. In principle, at each stage of the algorithm, the average error probability should be computed for any of the candidates codebooks. A possible efficient implementation may compute these error probabilities based on a pairwise error array computed for the initial codebook $C_{0}=\left\{x_{1}, \ldots, x_{m_{0}}\right\}$ and the given noise samples.

Specifically, consider the array $E \in\left\{2^{[n]}\right\}^{m_{0} \times m_{0}}$ where $2^{[n]}$ is the power set of $[n]$, such that the $\left(j_{1}, j_{2}\right)$ th entry of $E$ is given by

$$
E\left(j_{1}, j_{2}\right):=\left\{i:\left\|x_{j_{1}}-x_{j_{2}}\right\|_{S}^{2}+2\left(x_{j_{1}}-x_{j_{2}}\right)^{T} S z_{i}<0\right\}
$$

To wit, this entry $E\left(j_{1}, j_{2}\right)$ is the set of noise samples indices such that if for a codebook consisting only the codewords $\left(x_{j_{1}}, x_{j_{2}}\right)$, the noise sample $z_{i}$ will cause a decoding error when $x_{j_{1}}$ is transmitted. In accordance, $\bigcup_{j_{2} \in\left[m_{0}\right]}\left\{E\left(j_{1}, j_{2}\right)\right\} \subset[n]$ is the set of noise sample indices such that a decoding error occurs when $x_{j_{1}}$ is transmitted and the codebook is $C_{0}$. The array $E$ can be computed once at initialization of the algorithm, and then the error probabilities required by Algorithm 2 can be computed based only on this array. At first, for $C_{0}=\left\{x_{1}, \ldots, x_{m_{0}}\right\}$, the error probability is given by

$$
\mathbf{p}_{\boldsymbol{z}}\left(C_{0}\right)=\frac{1}{n} \sum_{i=1}^{n} \frac{1}{m_{0}} \sum_{j_{1}=1}^{m_{0}}\left|\bigcup_{j_{2} \in\left[m_{0}\right]}\left\{E\left(j_{1}, j_{2}\right)\right\}\right| .
$$

The error probability $\mathbf{p}_{\boldsymbol{z}}\left(C_{1}\right)$ for $C_{1}=C_{0} \backslash\left(x_{j_{1}}, \ldots, x_{j_{k}}\right)$ from $C_{0}$ can be computed by first removing the rows indexed by $\left(j_{1}, \ldots, j_{k}\right)$ and the rows indexed by $\left(j_{1}, \ldots, j_{k}\right)$ from the array $E\left(j_{1}, j_{2}\right)$ and then compute as in 290) with the new array. The main advantage of this approach is that the array tend to be sparse in the sense that either $\left|E\left(j_{1}, j_{2}\right)\right|$ is typically low - when transmitting $x_{j}$, there is only a relatively a small number of noise samples that will cause an error. This array become sparser with the steps of the algorithm, and moreover, high signal-to-noise ratio and high dimension lead to sparser arrays.

\section{REFERENCES}

[1] András Antos. Improved minimax bounds on the test and training distortion of empirically designed vector quantizers. IEEE Transactions on Information Theory, 51(11):4022-4032, 2005.

[2] András Antos, László Gyorfi, and Andras Gyorgy. Individual convergence rates in empirical vector quantizer design. IEEE Transactions on Information Theory, 51(11):4013-4022, 2005.

[3] Suguru Arimoto. An algorithm for computing the capacity of arbitrary discrete memoryless channels. IEEE Transactions on Information Theory, 18(1):14-20, 1972.

[4] Vladimir B. Balakirsky. A converse coding theorem for mismatched decoding at the output of binary-input memoryless channels. IEEE Transactions on Information Theory, 41(6):1889-1902, 1995.

[5] John R. Barry, Edward A. Lee, and David G. Messerschmitt. Digital communication. Springer Science \& Business Media, 2012.

[6] Peter L. Bartlett, Tamás Linder, and Gábor Lugosi. The minimax distortion redundancy in empirical quantizer design. IEEE Transactions on Information theory, 44(5):1802-1813, 1998. 
[7] Peter L. Bartlett and Shahar Mendelson. Rademacher and Gaussian complexities: Risk bounds and structural results. Journal of Machine Learning Research, 3(Nov):463-482, 2002.

[8] Mikhail Belkin, Siyuan Ma, and Soumik Mandal. To understand deep learning we need to understand kernel learning. In International Conference on Machine Learning, pages 541-549. PMLR, 2018.

[9] Richard Blahut. Computation of channel capacity and rate-distortion functions. IEEE transactions on Information Theory, 18(4):460473, 1972.

[10] Anselm Blumer, Andrzej Ehrenfeucht, David Haussler, and Manfred K. Warmuth. Learnability and the Vapnik-Chervonenkis dimension. Journal of the ACM (JACM), 36(4):929-965, 1989.

[11] William M. Bolstad. Understanding computational Bayesian statistics, volume 644. John Wiley \& Sons, 2009.

[12] Stéphane Boucheron, Gábor Lugosi, and Pascal Massart. Concentration inequalities: A nonasymptotic theory of independence. Oxford university press, 2013.

[13] Olivier Bousquet and André Elisseeff. Stability and generalization. Journal of machine learning research, 2(Mar):499-526, 2002.

[14] Stephen P. Boyd and Lieven Vandenberghe. Convex Optimization. Cambridge university press, 2004.

[15] Maurizio Bruglieri, Matthias Ehrgott, Horst W. Hamacher, and Francesco Maffioli. An annotated bibliography of combinatorial optimization problems with fixed cardinality constraints. Discrete Applied Mathematics, 154(9):1344-1357, 2006.

[16] William R. Caid and Robert W. Means. Neural network error correcting decoders for block and convolutional codes. In 1990 IEEE Global Telecommunications Conference and Exhibition, pages 1028-1031. IEEE, 1990.

[17] Thomas M. Cover and Joy A. Thomas. Elements of information theory. John Wiley \& Sons, 2012.

[18] Monika Csikos, Andrey Kupavskii, and Nabil H. Mustafa. Optimal bounds on the VC-dimension. arXiv preprint arXiv:1807.07924, 2018.

[19] Imre Csiszár and János Korner. Graph decomposition: A new key to coding theorems. IEEE Transactions on Information Theory, 27(1):5-12, 1981.

[20] Imre Csiszár and János Körner. Information Theory: Coding Theorems for Discrete Memoryless Systems. Cambridge University Press, Cambridge, U.K., 2011.

[21] Imre Csiszár and Prakash Narayan. Channel capacity for a given decoding metric. IEEE Transactions on Information Theory, 41(1):35-43, 1995.

[22] Imre Csiszár and Paul C. Shields. Information theory and statistics: A tutorial. Foundations and Trends® in Communications and Information Theory, 1(4):417-528, 2004.

[23] Steffen Dereich, Michael Scheutzow, and Reik Schottstedt. Constructive quantization: Approximation by empirical measures. In Annales de l'IHP Probabilités et statistiques, volume 49, pages 1183-1203, 2013.

[24] Suhas N. Diggavi and Thomas M. Cover. The worst additive noise under a covariance constraint. IEEE Transactions on Information Theory, 47(7):3072-3081, 2001.

[25] Cynthia Dwork, Vitaly Feldman, Moritz Hardt, Toni Pitassi, Omer Reingold, and Aaron Roth. Generalization in adaptive data analysis and holdout reuse. In Advances in Neural Information Processing Systems, pages 2350-2358, 2015.

[26] Cynthia Dwork, Vitaly Feldman, Moritz Hardt, Toniann Pitassi, Omer Reingold, and Aaron Roth. Preserving statistical validity in adaptive data analysis. In Proceedings of the forty-seventh annual ACM symposium on Theory of computing, pages 117-126, 2015.

[27] Cynthia Dwork and Aaron Roth. The algorithmic foundations of differential privacy. Foundations and Trends® in Theoretical Computer Science, 9(3-4):211-407, 2014.

[28] Alex Dytso, Semih Yagli, H. Vincent Poor, and Shlomo Shamai (Shitz). The capacity achieving distribution for the amplitude constrained additive Gaussian channel: An upper bound on the number of mass points. IEEE Transactions on Information Theory, pages $1-1,2019$.

[29] Matthias Ehrgott. Optimization problems in graphs under cardinality restrictions. Master's Thesis, Universität Kaiserslautern, Fachbereich Mathematik, 55, 1992. 
[30] Amedeo Roberto Esposito, Michael Gastpar, and Ibrahim Issa. Generalization error bounds via Rényi-, $f$-divergences and maximal leakage. arXiv preprint arXiv:1912.01439, 2019.

[31] Nariman Farsad and Andrea Goldsmith. Detection algorithms for communication systems using deep learning. arXiv preprint arXiv:1705.08044, 2017.

[32] Meir Feder and Neri Merhav. Universal composite hypothesis testing: A competitive minimax approach. IEEE Transactions on Information Theory, 48(6):1504-1517, June 2002.

[33] Vitaly Feldman and Jan Vondrak. Generalization bounds for uniformly stable algorithms. In Advances in Neural Information Processing Systems, pages 9769-9779, 2018.

[34] Vitaly Feldman and Jan Vondrak. High probability generalization bounds for uniformly stable algorithms with nearly optimal rate. In Conference on Learning Theory, pages 1270-1279. PMLR, 2019.

[35] G. David Forney. 6.451 principles of digital communication ii. https://ocw.mit.edu/courses/ electrical-engineering-and-computer-science/6-451-principles-of-digital-communication-ii-spring-2005/readings-and-lecture-notes/ MIT6_451S05_FullLecNotes.pdf. 2005.

[36] G. David Forney and Lee-Fang Wei. Multidimensional constellations. I. introduction, figures of merit, and generalized cross constellations. IEEE journal on selected areas in communications, 7(6):877-892, 1989.

[37] Nicolas Fournier and Arnaud Guillin. On the rate of convergence in Wasserstein distance of the empirical measure. Probability Theory and Related Fields, 162(3-4):707-738, 2015.

[38] Robert G. Gallager. A simple derivation of the coding theorem and some applications. IEEE Transactions on Information Theory, 11(1):3-18, 1965.

[39] Anand Ganti, Amos Lapidoth, and I. Emre Telatar. Mismatched decoding revisited: General alphabets, channels with memory, and the wide-band limit. IEEE Transactions on Information Theory, 46(7):2315-2328, 2000.

[40] Ziv Goldfeld, Kristjan Greenewald, Jonathan Niles-Weed, and Yury Polyanskiy. Convergence of smoothed empirical measures with applications to entropy estimation. IEEE Transactions on Information Theory, 2020.

[41] Ian Goodfellow, Yoshua Bengio, and Aaron Courville. Deep Learning. MIT Press, 2016.

[42] Robert M. Gray. Entropy and information theory. Springer Science \& Business Media, 2011.

[43] Robert M. Gray and David L. Neuhoff. Quantization. IEEE transactions on information theory, 44(6):2325-2383, 1998.

[44] Tobias Gruber, Sebastian Cammerer, Jakob Hoydis, and Stephan ten Brink. On deep learning-based channel decoding. In $201751 s t$ Annual Conference on Information Sciences and Systems (CISS), pages 1-6. IEEE, 2017.

[45] Asela Gunawardana and William Byrne. Convergence theorems for generalized alternating minimization procedures. Journal of machine learning research, 6(Dec):2049-2073, 2005.

[46] Yanjun Han, Jiantao Jiao, Tsachy Weissman, and Yihong Wu. Optimal rates of entropy estimation over Lipschitz balls. The Annals of Statistics, 48(6):3228-3250, 2020.

[47] Moritz Hardt, Ben Recht, and Yoram Singer. Train faster, generalize better: Stability of stochastic gradient descent. In International Conference on Machine Learning, pages 1225-1234. PMLR, 2016.

[48] Babak Hassibi and Bertrand M. Hochwald. How much training is needed in multiple-antenna wireless links? IEEE Transactions on Information Theory, 49(4):951-963, 2003.

[49] Trevor Hastie, Robert Tibshirani, and Jerome Friedman. The elements of statistical learning: Data mining, inference, and prediction. Springer Science \& Business Media, 2009.

[50] W. Keith Hastings. Monte Carlo sampling methods using Markov chains and their applications. 1970.

[51] Robert W. Heath Jr. and Angel Lozano. Foundations of MIMO Communication. Cambridge University Press, 2018.

[52] Kurt Hornik, Maxwell Stinchcombe, and Halbert White. Multilayer feedforward networks are universal approximators. Neural networks, 2(5):359-366, 1989.

[53] Jianyi Huang and Sean P. Meyn. Characterization and computation of optimal distributions for channel coding. IEEE Transactions on Information Theory, 51(7):2336-2351, 2005. 
[54] Joseph Yu Ngai Hui. Fundamental issues of multiple accessing. PhD thesis, Massachusetts Institute of Technology, 1983.

[55] Wasim Huleihel, Salman Salamatian, Neri Merhav, and Muriel Médard. Gaussian intersymbol interference channels with mismatch. IEEE Transactions on Information Theory, 65(7):4499-4517, 2019.

[56] Mohamed Ibnkahla. Applications of neural networks to digital communications - a survey. Signal processing, 80(7):1185-1215, 2000.

[57] Shunsuke Ihara. On the capacity of channels with additive non-Gaussian noise. Information and Control, 37(1):34-39, 1978.

[58] Yihan Jiang, Hyeji Kim, Himanshu Asnani, Sreeram Kannan, Sewoong Oh, and Pramod Viswanath. Learn codes: Inventing lowlatency codes via recurrent neural networks. In ICC 2019-2019 IEEE International Conference on Communications (ICC), pages 1-7. IEEE, 2019.

[59] David S. Johnson, Cecilia R. Aragon, Lyle A. McGeoch, and Catherine Schevon. Optimization by simulated annealing: An experimental evaluation; part I, graph partitioning. Operations research, 37(6):865-892, 1989.

[60] Hyeji Kim, Yihan Jiang, Sreeram Kannan, Sewoong Oh, and Pramod Viswanath. Deepcode: Feedback codes via deep learning. In Advances in Neural Information Processing Systems, pages 9436-9446, 2018.

[61] Hyeji Kim, Yihan Jiang, Ranvir Rana, Sreeram Kannan, Sewoong Oh, and Pramod Viswanath. Communication algorithms via deep learning. arXiv preprint arXiv:1805.09317, 2018.

[62] Scott Kirkpatrick, C. Daniel Gelatt, and Mario P. Vecchi. Optimization by simulated annealing. science, 220(4598):671-680, 1983.

[63] Amos Lapidoth. Nearest neighbor decoding for additive non-Gaussian noise channels. IEEE Transactions on Information Theory, 42(5):1520-1529, 1996.

[64] Amos Lapidoth. A foundation in digital communication. Cambridge University Press, 2017.

[65] Amos Lapidoth and Prakash Narayan. Reliable communication under channel uncertainty. IEEE Transactions on Information Theory, 44(6):2148-2177, 1998

[66] Jaeho Lee and Maxim Raginsky. Learning finite-dimensional coding schemes with nonlinear reconstruction maps. SIAM Journal on Mathematics of Data Science, 1(3):617-642, 2019.

[67] Clément Levrard. Fast rates for empirical vector quantization. Electronic Journal of Statistics, 7:1716-1746, 2013.

[68] Clément Levrard. Nonasymptotic bounds for vector quantization in hilbert spaces. The Annals of Statistics, 43(2):592-619, 2015.

[69] Tengyuan Liang, Alexander Rakhlin, and Karthik Sridharan. Learning with square loss: Localization through offset Rademacher complexity. In Conference on Learning Theory, pages 1260-1285, 2015.

[70] Tamás Linder. On the training distortion of vector quantizers. IEEE Transactions on Information Theory, 46(4):1617-1623, 2000.

[71] Tamás Linder. Learning-theoretic methods in vector quantization. In Principles of nonparametric learning, pages 163-210. Springer, 2002.

[72] Tamás Linder, Gábor Lugosi, and Kenneth Zeger. Rates of convergence in the source coding theorem, in empirical quantizer design, and in universal lossy source coding. IEEE Transactions on Information Theory, 40(6):1728-1740, 1994.

[73] Tamás Linder, Gábor Lugosi, and Kenneth Zeger. Empirical quantizer design in the presence of source noise or channel noise. IEEE Transactions on Information Theory, 43(2):612-623, 1997.

[74] Katalin Marton. Bounding $\bar{d}$-distance by informational divergence: A method to prove measure concentration. Annals of probability, 24(2):857-866, 1996.

[75] Thomas L. Marzetta. Massive MIMO: An introduction. Bell Labs Technical Journal, 20:11-22, 2015.

[76] Andreas Maurer and Massimiliano Pontil. $k$-dimensional coding schemes in Hilbert spaces. IEEE Transactions on Information Theory, 56(11):5839-5846, 2010.

[77] Shahar Mendelson. Learning without concentration. In Conference on Learning Theory, pages 25-39, 2014.

[78] Shahar Mendelson. Learning without concentration for general loss functions. Probability Theory and Related Fields, 171(1):459-502, 2018.

[79] Neri Merhav. Universal decoding for memoryless Gaussian channels with a deterministic interference. IEEE Transactions on Information Theory, 39(4):1261-1269, 1993. 
[80] Neri Merhav. How many information bits does a decoder need about the channel statistics? IEEE Transactions on Information Theory, 43(5):1707-1714, 1997.

[81] Neri Merhav. Universal decoding for arbitrary channels relative to a given class of decoding metrics. IEEE Transactions on Information Theory, 59(9):5566-5576, 2013.

[82] Neri Merhav, Gideon Kaplan, Amos Lapidoth, and S. Shamai-Shitz. On information rates for mismatched decoders. IEEE Transactions on Information Theory, 40(6):1953-1967, 1994.

[83] Neri Merhav and Jacob Ziv. On the amount of statistical side information required for lossy data compression. IEEE Transactions on Information Theory, 43(4):1112-1121, 1997.

[84] Nicholas Metropolis, Arianna W. Rosenbluth, Marshall N. Rosenbluth, Augusta H. Teller, and Edward Teller. Equation of state calculations by fast computing machines. The journal of chemical physics, 21(6):1087-1092, 1953.

[85] Urbashi Mitra and H. Vincent Poor. Neural network techniques for adaptive multiuser demodulation. IEEE Journal on Selected Areas in Communications, 12(9):1460-1470, 1994.

[86] K. Moshksar and A. K. Khandani. Arbitrarily tight bounds on differential entropy of Gaussian mixtures. IEEE Transactions on Information Theory, 62(6):3340-3354, June 2016.

[87] Eliya Nachmani, Elad Marciano, Loren Lugosch, Warren J. Gross, David Burshtein, and Yair Be'ery. Deep learning methods for improved decoding of linear codes. IEEE Journal of Selected Topics in Signal Processing, 12(1):119-131, 2018.

[88] Timothy O'Shea and Jakob Hoydis. An introduction to deep learning for the physical layer. IEEE Transactions on Cognitive Communications and Networking, 3(4):563-575, 2017.

[89] Lawrence H. Ozarow and Aaron D. Wyner. On the capacity of the Gaussian channel with a finite number of input levels. IEEE transactions on information theory, 36(6):1426-1428, 1990.

[90] Mark S. Pinsker. Calculation of the rate of information production by means of stationary random processes and the capacity of stationary channel. In Dokl. Akad. Nauk USSR, volume 111, pages 753-756, 1956.

[91] David Pollard. Quantization and the method of $k$-means. IEEE Transactions on Information theory, 28(2):199-205, 1982.

[92] Yury Polyanskiy and Yihong Wu. Wasserstein continuity of entropy and outer bounds for interference channels. IEEE Transactions on Information Theory, 62(7):3992-4002, 2016.

[93] John G. Proakis and Masoud Salehi. Digital communications, volume 4. McGraw-hill New York, 2001.

[94] Maxim Raginsky, Alexander Rakhlin, Matthew Tsao, Yihong Wu, and Aolin Xu. Information-theoretic analysis of stability and bias of learning algorithms. In 2016 IEEE Information Theory Workshop (ITW), pages 26-30. IEEE, 2016.

[95] Maxim Raginsky, Alexander Rakhlin, and Aolin Xu. Information-theoretic stability and generalization. In M. R. D. Rodrigues and Y. C. Eldar, editors, Information-Theoretic Methods in Data Science, chapter 10. Cambridge University Press, Cambridge, 2020.

[96] Maxim Raginsky and Igal Sason. Concentration of Measure Inequalities in Information Theory, Communications, and Coding. Now Foundations and Trends, 2018.

[97] Alexander Rakhlin and Karthik Sridharan. Statistical learning theory and sequential prediction. Lecture Notes in University of Pennsyvania, 2012.

[98] Borzoo Rassouli and Bruno Clerckx. On the capacity of vector Gaussian channels with bounded inputs. IEEE Transactions on Information Theory, 62(12):6884-6903, 2016.

[99] Tom Richardson and Ruediger Urbanke. Modern coding theory. Cambridge university press, 2008.

[100] Michael Sabin and Robert Gray. Global convergence and empirical consistency of the generalized Lloyd algorithm. IEEE Transactions on information theory, 32(2):148-155, 1986.

[101] Anant Sahai, Joshua Sanz, Vignesh Subramanian, Caryn Tran, and Kailas Vodrahalli. Learning to communicate in a noisy environment. arXiv preprint arXiv:1910.09630, 2019.

[102] Neev Samuel, Tzvi Diskin, and Ami Wiesel. Deep MIMO detection. In 2017 IEEE 18th International Workshop on Signal Processing Advances in Wireless Communications (SPAWC), pages 1-5. IEEE, 2017. 
[103] Jonathan Scarlett, Albert Guillén i Fàbregas, Anelia Somekh-Baruch, and Alfonso Martinez. Information-theoretic foundations of mismatched decoding. Foundations and Trends in Communications and Information Theory, 17(2-3):149-401, 2020.

[104] Tim Schenk. RF imperfections in high-rate wireless systems: Impact and digital compensation. Springer Science \& Business Media, 2008.

[105] Shai Shalev-Shwartz and Shai Ben-David. Understanding machine learning: From theory to algorithms. Cambridge university press, 2014.

[106] Shlomo Shamai (Shitz) and Israel Bar-David. The capacity of average and peak-power-limited quadrature Gaussian channels. IEEE Transactions on Information Theory, 41(4):1060-1071, 1995.

[107] Joel G. Smith. The information capacity of amplitude-and variance-constrained sclar Gaussian channels. Information and control, 18(3):203-219, 1971

[108] Anelia Somekh-Baruch. On achievable rates and error exponents for channels with mismatched decoding. IEEE Transactions on Information Theory, 61(2):727-740, 2014.

[109] Anelia Somekh-Baruch. A general formula for the mismatch capacity. IEEE Transactions on Information Theory, 61(9):4554-4568, 2015.

[110] Michal Sybis, Krzysztof Wesolowski, Keeth Jayasinghe, Venkatkumar Venkatasubramanian, and Vladimir Vukadinovic. Channel coding for ultra-reliable low-latency communication in 5G systems. In 2016 IEEE 84th vehicular technology conference (VTC-Fall), pages 1-5. IEEE, 2016.

[111] David Tse and Pramod Viswanath. Fundamentals of wireless communication. Cambridge university press, 2005.

[112] Alexandre B. Tsybakov. Introduction to nonparametric estimation. Springer Science \& Business Media, 2008.

[113] Daniel Vainsencher, Shie Mannor, and Alfred M. Bruckstein. The sample complexity of dictionary learning. Journal of Machine Learning Research, 12(Nov):3259-3281, 2011.

[114] Tim Van Erven and Peter Harremos. Rényi divergence and Kullback-Leibler divergence. IEEE Transactions on Information Theory, 60(7):3797-3820, 2014

[115] Ramon van Handel. Probability in high dimension. Technical report, Princeton University New Jersey, 2016.

[116] Vladimir Vapnik. The nature of statistical learning theory. Springer science \& business media, 2013.

[117] Sergio Verdú and Tsachy Weissman. The information lost in erasures. IEEE Transactions on Information Theory, 54(11):5030-5058, 2008 .

[118] Roman Vershynin. High-dimensional probability: An introduction with applications in data science, volume 47. Cambridge University Press, 2018

[119] Andrew J. Viterbi and Jim K. Omura. Principles of digital communication and coding. Courier Corporation, 2013.

[120] Qing Wang, Sanjeev R. Kulkarni, and Sergio Verdú. Universal estimation of information measures for analog sources. Foundations and Trends ${ }^{\circledR}$ in Communications and Information Theory, 5(3):265-353, 2009.

[121] Tianqi Wang, Chao-Kai Wen, Hanqing Wang, Feifei Gao, Tao Jiang, and Shi Jin. Deep learning for wireless physical layer: Opportunities and challenges. China Communications, 14(11):92-111, 2017.

[122] Xiao-An Wang and Stephen B. Wicker. An artificial neural net Viterbi decoder. IEEE Transactions on communications, 44(2):165-171, 1996.

[123] Nir Weinberger and Meir Feder. Universal decoding for linear Gaussian fading channels in the competitive minimax sense. In 2008 IEEE International Symposium on Information Theory, pages 782-786. IEEE, 2008.

[124] Nir Weinberger and Meir Feder. $k$-vectors: An alternating minimization algorithm for learning regression functions. IEEE Transactions on Information Theory, 66(11):7196-7221, 2020.

[125] C. F. Jeff Wu. On the convergence properties of the EM algorithm. The Annals of statistics, 11(1):95-103, 1983.

[126] Yihong Wu and Sergio Verdú. The impact of constellation cardinality on Gaussian channel capacity. In 2010 48th Annual Allerton Conference on Communication, Control, and Computing (Allerton), pages 620-628. IEEE, 2010. 
[127] Aaron D. Wyner, Jacob Ziv, and Abraham J. Wyner. On the role of pattern matching in information theory. IEEE Transactions on information Theory, 44(6):2045-2056, 1998.

[128] Aolin Xu and Maxim Raginsky. Information-theoretic analysis of generalization capability of learning algorithms. In Advances in Neural Information Processing Systems, pages 2524-2533, 2017.

[129] Ram Zamir. Lattice Coding for Signals and Networks: A Structured Coding Approach to Quantization, Modulation, and Multiuser Information Theory. Cambridge University Press, 2014.

[130] Willard I. Zangwill. Convergence conditions for nonlinear programming algorithms. Management Science, 16(1):1-13, 1969.

[131] Wenyi Zhang, Yizhu Wang, Cong Shen, and Ning Liang. A regression approach to certain information transmission problems. IEEE Journal on Selected Areas in Communications, 37(11):2517-2531, 2019.

[132] Jacob Ziv and Abraham Lempel. A universal algorithm for sequential data compression. IEEE Transactions on information theory, 23(3):337-343, 1977.

[133] Jacob Ziv and Abraham Lempel. Compression of individual sequences via variable-rate coding. IEEE transactions on Information Theory, 24(5):530-536, 1978 